\title{
Some Limit Transitions between $B C$ Type Orthogonal Polynomials Interpreted on Quantum Complex Grassmannians
}

\author{
By \\ Mathijs S. DiJkhuizen * and Jasper V. Stokman ${ }^{* *}$
}

\begin{abstract}
The quantum complex Grassmannian $U_{q} / K_{q}$ of rank $l$ is the quotient of the quantum unitary group $U_{q}=U_{q}(n)$ by the quantum subgroup $K_{q}=U_{q}(n-l) \times U_{q}(l)$. We show that $\left(U_{q}, K_{q}\right)$ is a quantum Gelfand pair and we express the zonal spherical functions, i.e. $K_{q}$-biinvariant matrix coefficients of finite-dimensional irreducible representations of $U_{q}$, as multivariable little $q$-Jacobi polynomials depending on one discrete parameter. Another type of biinvariant matrix coefficients is identified as multivariable big $q$-Jacobi polynomials. The proof is based on earlier results by Noumi, Sugitani and the first author relating Koornwinder polynomials to a one-parameter family of quantum complex Grassmannians, and certain limit transitions from Koornwinder polynomials to multivariable big and little $q$-Jacobi polynomials studied by Koornwinder and the second author.
\end{abstract}

\section{§1. Introduction}

The first connection between $q$-special functions and quantum groups was revealed in the late 1980's by the interpretation of little $q$-Jacobi polynomials as matrix coefficients of irreducible representations of the quantum $S U(2)$ group (cf. $[\mathrm{VS} 1],[\mathrm{Ms}],[\mathrm{K} 1])$. In the past decade, many other connections between representation theory of quantum groups and the theory of $q$-special functions have been discovered.

For instance, Noumi and Mimachi $[\mathrm{NM}]$ showed that the zonal spherical functions on Podles̀'s one-parameter family of quantum 2-spheres can be identified

Communicated by T. Miwa, July 2, 1998.

1991 Mathematics Subject Classification: 33D80, 33D45, 17B37, 81R50.

* Department of Mathematics, Faculty of Science, Kobe University, Rokko, Kobe 657, Japan.

** Korteweg-de Vries institute for mathematics, University of Amsterdam, Plantage Muidergracht 24, 1018 TV Amsterdam, The Netherlands. 
with big $q$-Jacobi polynomials. In $[\mathrm{K} 2]$ and $[\mathrm{K} 4]$, Koornwinder generalized these results by replacing the notion of invariance under a quantum subgroup by the notion of invariance under a twisted primitive element in the quantized universal enveloping algebra. In particular, this infinitesimal approach allowed Koornwinder to identify the zonal spherical functions on Podles̀ spheres as a two-parameter family of Askey-Wilson polynomials. Noumi's and Mimachi's results [NM] could then be reobtained by sending one parameter to infinity. If both parameters are sent to infinity, one obtains the interpretation of little $q$-Jacobi polynomials as zonal spherical functions on the "standard" 2 -sphere, i.e. the quantum 2 -sphere which is realized as the quotient of the quantum $S U(2)$ group by the standard maximal torus. On the level of $q$-special functions, these limits correspond to certain explicit limit transitions from Askey-Wilson polynomials to big respectively little $q$-Jacobi polynomials.

Analogous statements are valid for complex projective space, see Noumi, Yamada and Mimachi [NYM] for the little $q$-Jacobi case, and Dijkhuizen and Noumi [DN1] for the general case. In this paper we generalize these results to the higher rank setting by interpreting certain subfamilies of Koornwinder's multivariable analogues of the Askey-Wilson polynomials and certain subfamilies of the multivariable big and little $q$-Jacobi polynomials (cf. [S1]) as zonal spherical functions on quantum analogues of the complex Grassmannian

$$
U / K:=U(n) /(U(n-l) \times U(l)), \quad(l \leq[n / 2]),
$$

where $U(n)$ is the group of $n \times n$ unitary matrices.

Koornwinder's infinitesimal approach to harmonic analysis on quantized symmetric spaces was for the first time successfully generalized to higher rank cases by Noumi $[\mathrm{N}]$. The quantized symmetric spaces were now defined using invariance under certain two-sided coideals in the quantized universal enveloping algebra. So far, this method has been successfully applied to all compact symmetric spaces of classical type, e.g. $[\mathrm{NS} 1],[\mathrm{NS} 2],[\mathrm{NDS}],[\mathrm{Su}],[\mathrm{DN} 1]$. The related zonal spherical functions can all be identified with Koornwinder polynomials or Macdonald polynomials.

In particular, Noumi, Sugitani and the first author [NDS] introduced a oneparameter family of quantum analogues of the complex Grassmannian. They announced that the spherical functions associated with these quantized symmetric spaces can be expressed as a two-parameter subfamily of the Koornwinder polynomials.

In this paper we extend these results to the quantum subgroup case, i.e. we determine the zonal spherical functions associated with the quantum analogue of the complex Grassmannian (denoted by $U_{q} / K_{q}$ ) which is defined as the quotient of the quantum unitary group $U_{q}(n)$ by the obvious quantum subgroup $K_{q}$ corresponding to $K=U(n-l) \times U(l)$. We show that the quantum space $U_{q} / K_{q}$ can be 
formally obtained from the one-parameter family of quantum Grassmannians defined in [NDS] by sending the parameter to infinity. We also show that this limit transition on quantum Grassmannians is compatible with the limit transitions from Koornwinder polynomials to multivariable big and little $q$-Jacobi polynomials, which were previously studied by Koornwinder and the second author [SK].

In order to give a rigorous meaning to the above-mentioned formal limit between quantum Grassmannians we use the recent result of the second author [S4] that the limits from Koornwinder polynomials to multivariable big and little $q$-Jacobi polynomials can be taken on the level of the orthogonality measures (in a suitable weak sense). Indeed, this result allows us to rigorously compute the limit of the quantum Schur orthogonality relations for the zonal spherical functions. This in turn leads to a complete description of the harmonic analysis on the quantum Grassmannian $U_{q} / K_{q}$. In particular, we obtain an identification of the zonal spherical functions on the quantum complex Grassmannian $U_{q} / K_{q}$ with multivariable big respectively little $q$-Jacobi polynomials.

We remark that in the rank one case, there are several alternative methods for determining the zonal spherical functions in the quantum subgroup case (see e.g. $[\mathrm{NYM}],[\mathrm{VS} 2]$ ). However, the relatively indirect method using limit arguments seems to be the only method which admits a direct generalization to the higher rank cases of the complex Grassmannian.

There are strong indications that such limit arguments can also be applied for other quantum compact symmetric spaces. Indeed, an important prerequisite for applying such limit arguments is the existence of a suitable one-parameter family of quantizations of the compact symmetric space under consideration. The occurrence of such a one-parameter phenomena on the quantum level is directly related to the existence of a one-parameter family of covariant Poisson brackets on the underlying symmetric space. The existence of such a one-parameter family of covariant Poisson brackets in the case of a Hermitian symmetric space was established by Khoroshkin \& Radul \& Rubtsov [KRR] and Donin \& Gurevich $[\mathrm{DG}]$ (see also $[\mathrm{Dz}]$ for more information and references).

The paper is organized as follows. In $\$ 2$ we briefly recall some results on the structure of the (classical) complex Grassmannian $U / K$ and on the nature of its zonal spherical functions. In $\S 3$ we recall the definition and the main properties of the Koornwinder polynomials and the multivariable big and little $q$-Jacobi polynomials. In $\S 4$ we collect some facts about the quantum unitary group that will be heavily used in later sections. In $\$ 5$ we determine the spherical dominant weights for the quantum complex Grassmannian $U_{q} / K_{q}$. In $\S 6$ we recall the one-parameter family of quantum Grassmannians and determine the corresponding spherical weights. In $\$ 7$ we express the zonal spherical functions on the one-parameter family of quantum Grassmannians as a subfamily of the Koornwinder polynomials. Many of the results in $\S 6$ and $\S 7$, previously announced in [NDS], are proved here in full detail using so-called "principal term" type of arguments, cf. [NS2], [Su]. 
Finally, in $\$ 8$ we study the limit from the one-parameter family of quantum Grassmannians to $U_{q} / K_{q}$, which in particular leads to the interpretation of multivariable big and little $q$-Jacobi polynomials as zonal spherical functions on the quantum Grassmannian $U_{q} / K_{q}$.

Throughout this paper we will use the convention that vector spaces are defined over the complex numbers and that algebras have a unit element.

\section{§2. The Classical Complex Grassmannian}

Two general references for the contents of this section are Helgason $[\mathrm{He}]$, and Heckman and Schlichtkrull [HS].

Throughout this paper, $n \geq 2$ and $1 \leq l \leq\left[\frac{n}{2}\right]$ are fixed integers. Let $G:=G L(n$, $\mathbb{C})$ denote the general linear group with Lie algebra $\mathfrak{g}=\mathrm{gl}(n, \mathbb{C})$, and $U:=U(n)$ the unitary group with Lie algebra $\mathfrak{u}$. Let $\mathbb{T} \subset U$ denote the maximal torus consisting of diagonal matrices in $U$. Write $\mathfrak{h} \subset \mathfrak{g}$ for the corresponding Cartan subalgebra. Let $e_{i j}(1 \leq i, j \leq n)$ denote the standard matrix units. The matrices $h_{i}:=e_{i i}(1 \leq i \leq n)$ form a basis of $\mathfrak{h}$. Write $\tilde{\varepsilon}_{i} \in \mathfrak{h}^{*}(1 \leq i \leq n)$ for the corresponding dual basis vectors, and define a non-degenerate symmetric bilinear form on $\mathfrak{h}^{*}$ by $\left\langle\tilde{\varepsilon}_{i}, \tilde{\varepsilon}_{j}\right\rangle=\delta_{i j}$. The usual positive system $R^{+}$in the root system $R:=R(\mathfrak{g}, \mathfrak{h})$ consists of the vectors $\tilde{\varepsilon}_{i}-\tilde{\varepsilon}_{j}(1 \leq i<j \leq n)$. Let $\mathbb{P}=P_{n}:=\bigoplus_{1 \leq i \leq n} \mathbb{Z} \tilde{\varepsilon}_{i}$ denote the rational character lattice of $G$ (equivalently, the lattice of analytically integral weights of $U$ ). Recall that the cone of dominant weights $P^{+}=P_{n}^{+}$is given by

$$
P_{n}^{+}:=\left\{\left(\lambda_{1}, \ldots, \lambda_{n}\right) \in P \mid \lambda_{1} \geq \lambda_{2} \geq \cdots \geq \lambda_{n}\right\}
$$

Denote by $\leq$ the (partial) dominance ordering on $P$. One has $\mu \leq \lambda$ if and only if

$$
\sum_{i=1}^{j} \mu_{l} \leq \sum_{i=1}^{j} \lambda_{l} \quad(1 \leq j \leq n-1) \quad \text { and } \quad \sum_{i=1}^{n} \mu_{i}=\sum_{i=1}^{n} \lambda_{i} .
$$

We write $K:=U(n-l) \times U(l)$ and $\mathfrak{f}:=\operatorname{gl}(n-l, \mathbb{C}) \oplus \operatorname{gl}(l, \mathbb{C})$ for the corresponding complexified Lie algebra $\mathfrak{f}$. $K$ is regarded as a subgroup of $U$ via the embedding

$$
U(n-l) \times U(l) \hookrightarrow U(n), \quad(A, B) \longmapsto\left(\begin{array}{ll}
A & 0 \\
0 & B
\end{array}\right) .
$$

The pair $(U, K)$ is symmetric. Indeed, the involutive Lie group automorphism $\theta: U \rightarrow U$ defined by $\theta(g):=J g J$ with

$$
J:=\sum_{1 \leq k \leq n-l} e_{k k}-\sum_{1 \leq k \leq l} e_{k^{\prime} k^{\prime}}, \quad\left(k^{\prime}:=n+1-k\right)
$$

has fixed point group $K$. The differential of $\theta$ at the unit element $e \in U$, extended 
$\mathbb{C}$-linearly to a Lie algebra involution of $\mathfrak{g}$, will also be denoted by $\theta$. The +1 eigenspace of the involution $\theta: \mathfrak{g} \rightarrow \mathfrak{g}$ is exactly the Lie subalgebra $\mathfrak{f}$. Writing $\mathfrak{p}$ for the -1 eigenspace of $\theta$ we have the eigenspace decomposition $\mathfrak{g}=\mathfrak{f} \oplus \mathfrak{p}$.

For certain purposes, it is more convenient to consider the involution $\theta^{\prime}: \mathfrak{g} \rightarrow$ $\mathfrak{g}$ defined by $\theta^{\prime}(X):=J^{\prime} X J^{\prime}$ with

$$
J^{\prime}:=\sum_{l<k<l^{\prime}} e_{k k}-\sum_{1 \leq k \leq l} e_{k k^{\prime}}-\sum_{1 \leq k \leq l} e_{k^{\prime} k} .
$$

Since $J^{\prime}$ is conjugate to $J$, the involution $\theta^{\prime}$ is conjugate to $\theta$ by an inner automorphism of $\mathfrak{g}$. Observe that both $\theta$ and $\theta^{\prime}$ leave $\mathfrak{u} \subset \mathfrak{g}$ invariant.

Let $\mathfrak{g}=\mathfrak{f}^{\prime} \oplus \mathfrak{p}^{\prime}$ be the eigenspace decomposition of $\theta^{\prime}$ into +1 and -1 eigenspaces. The intersection $\mathfrak{h} \cap \mathfrak{f}^{\prime}$ is spanned by the elements $h_{i}+h_{i^{\prime}}(1 \leq i \leq l)$ and $h_{i}(l$ $\left.<i<l^{\prime}\right)$, whereas the intersection $\mathfrak{a}:=\mathfrak{h} \cap \mathfrak{p}^{\prime}$ is spanned by $h_{i}-h_{i^{\prime}}(1 \leq i \leq l)$ and is maximal abelian in $\mathfrak{p}^{\prime}$. The positive system of $R$ taken with respect to the lexicographic ordering of $\mathfrak{h}_{\mathbb{R}}:=\sum_{j=1}^{n} \mathbb{R} h_{j}$ relative to the ordered basis $h_{1}-h_{1^{\prime}}, \ldots$, $h_{l}-h_{l^{\prime}}, h_{1}+h_{1^{\prime}}, \ldots, h_{l}+h_{l^{\prime}}, h_{l+1}, \ldots, h_{n-l}$ coincides with $R^{+}$.

Write $\varepsilon_{i}^{\prime}$ for the restriction of $\tilde{\varepsilon}_{i}$ to $\mathfrak{a}(1 \leq i \leq l)$. The root system $R \subset \mathfrak{h}^{*}$ is mapped under the natural projection $\mathfrak{h}^{*} \longrightarrow \mathfrak{a}^{*}$ onto the restricted root system $\Sigma^{\prime}$ $=\Sigma^{\prime}(\mathfrak{g}, \mathfrak{a})$. Choose the positive system in $\Sigma^{\prime}$ with respect to the lexicographic ordering of $\mathfrak{a}_{\mathbb{R}}:=\mathfrak{h}_{\mathbb{R}} \cap \mathfrak{a}$ relative to the ordered basis $h_{1}-h_{1^{\prime}}, \ldots, h_{l}-h_{l^{\prime}}$ of $\mathfrak{a}_{\mathbb{R}}$. This ordering is compatible with the lexicographic ordering of $\mathfrak{h}_{\mathbb{R}}$ introduced above in the sense that $\lambda \in \mathfrak{h}_{\mathbb{R}}^{*}$ is positive if its restriction to $\mathfrak{a}_{\mathbb{R}}$ is strictly positive. The positive root vectors in $\Sigma^{\prime}$ are

$$
\varepsilon_{i}^{\prime} \quad(1 \leq i \leq l), \quad \varepsilon_{i}^{\prime} \pm \varepsilon_{j}^{\prime} \quad(1 \leq i<j \leq l), \quad 2 \varepsilon_{i}^{\prime} \quad(1 \leq i \leq l),
$$

the roots $\varepsilon_{i}^{\prime}(1 \leq i \leq l)$ occurring only if $n \neq 2 l . \quad \Sigma^{\prime}$ is isomorphic with $B C_{l}$ if $n \neq 2 l$ and isomorphic with $C_{l}$ if $n=2 l$. The root multiplicities corresponding to the short, medium, and long roots are

$$
m_{1}=2(n-2 l), \quad m_{2}=2(l>1), \quad m_{3}=1 .
$$

For later purposes, it is convenient to rescale the root system $\Sigma^{\prime}$ by a factor 2 . So we see $\Sigma:=2 \Sigma^{\prime} \subset \mathfrak{a}^{*}, \varepsilon_{i}:=2 \varepsilon_{i}^{\prime}(1 \leq i \leq l)$. Then the corresponding weight lattice $P_{\Sigma} \subset \mathfrak{a}^{*}$ is the $\mathbb{Z}$-span of the $\varepsilon_{i}(1 \leq i \leq l)$, and the set $P_{\Sigma}^{+}$of dominant weights $\mu=$ $\sum_{i} \mu_{i} \varepsilon_{i}$ (taken with respect to the lexicographic ordering on $\mathfrak{a}_{\mathbb{R}}$ introduced above) is characterized by the condition $\mu_{1} \geq \cdots \geq \mu_{l} \geq 0$. The dominance ordering $\leq$ on $P_{\Sigma}$ is explicitly given by

$$
\mu \leq \lambda \Longleftrightarrow \sum_{i=1}^{J} \mu_{i} \leq \sum_{i=1}^{J} \lambda_{i} \quad(1 \leq j \leq l) .
$$

Let $K^{\prime} \subset U$ denote the connected subgroup corresponding to $\mathfrak{f}^{\prime}$. The symmet- 
ric pairs $(U, K)$ and $\left(U, K^{\prime}\right)$ are Gelfand pairs, i.e. every finite-dimensional irreducible representation of $U$ has at most one $K$-fixed vector up to scalar multiples. According to [He, Chapter V, Theorem 4.1], a highest weight $\lambda \in P^{+}$ is $K^{\prime}$-spherical, i.e. corresponds to a representation with a non-zero $K^{\prime}$-fixed vector, if and only if the restriction of $\lambda$ to $\mathfrak{h} \cap \mathfrak{f}^{\prime}$ is zero and the restriction of $\lambda$ to $a$ lies in $P_{\Sigma}^{+}$. Hence we get the following result.

Theorem 2.1. The set $P_{K}^{+} \subset P^{+}$of $K$-spherical dominant weights consists of all dominant weights of the form

$$
\lambda:=\left(\lambda_{1}, \ldots, \lambda_{l}, 0, \ldots, 0,-\lambda_{l}, \ldots,-\lambda_{1}\right)
$$

Write $\lambda^{\natural}:=\left(\lambda_{1}, \ldots, \lambda_{l}\right)$ for a dominant weight $\lambda \in P_{K}^{+}$. The assignment $\lambda \longmapsto$ $\lambda^{\natural}$ defines a bijection of $P_{K}^{+}$onto $P_{\Sigma}^{+}$. Let $\varpi_{r} \in P_{K}^{+}$be the spherical weight for which $\varpi_{r}^{\natural}=\left(1^{r}\right)$. Then $P_{K}^{+}=\oplus_{1 \leq r \leq l} \mathbb{Z}_{+} \varpi_{r}$. We will call $\left\{\varpi_{r}\right\}_{r=1}^{l}$ the fundamental dominant spherical weights.

Let $A$ denote the algebra of polynomial functions on $U, \mathrm{~A}(\mathbb{T})$ the algebra of polynomial functions on the maximal torus $\mathbb{T} . A(\mathbb{T})$ may be naturally identified with the algebra $\mathbb{C}\left[z^{ \pm 1}\right]=\mathbb{C}\left[z_{1}^{ \pm 1}, \ldots, z_{n}^{ \pm 1}\right]$ in $n$ variables $z_{i}(1 \leq i \leq n)$ in the following way. Observe that $\mathbb{T} \simeq i \mathfrak{h}_{\mathbb{R}} / 2 \pi i \widetilde{P}$ via the exponential mapping, where $\tilde{P}:=\oplus_{1 \leq j \leq n} \mathbb{Z} h_{j}$. Then the coordinate functions $z_{j}$ can be defined by $z_{j}:=e^{\tilde{\varepsilon}_{j}}$, where $e^{\tilde{\varepsilon}_{j}}([X]):=e^{\tilde{\varepsilon}_{j}(X)}$ for $[X] \in T$ with $X \in i \mathfrak{h}_{\mathbb{R}}$ a representative of $[X]$. More explicitly, $z_{j}$ is given by

$$
z_{j}: \operatorname{diag}\left(e^{i \theta_{1}}, e^{i \theta_{2}}, \ldots, e^{i \theta_{n}}\right) \longmapsto e^{i \theta_{j}}, \quad\left(\theta_{k} \in[0,2 \pi)\right)
$$

where diag $\left(a_{1}, \ldots, a_{n}\right)$ is the diagonal matrix with $a_{1}, \ldots, a_{n}$ on the diagonal.

Let $\mathscr{H} \subset A$ denote the subalgebra of $K^{\prime}$-biinvariant functions. One has the decomposition

$$
\mathscr{H}=\underset{\lambda \in P_{K}^{+}}{\bigoplus} \mathscr{H}(\lambda), \quad \mathscr{H}(\lambda):=\mathscr{H} \cap W(\lambda),
$$

$W(\lambda) \subset A$ denoting the subspace spanned by the matrix coefficients of the irreducible representation of highest weight $\lambda$. Each of the subspaces $\mathscr{H}(\lambda)\left(\lambda \in P_{K}^{+}\right)$ is one-dimensional, since $\left(U, K^{\prime}\right)$ is a Gelfand pair. Any non-zero element $\varphi(\lambda)$ of $\mathscr{H}(\lambda)$ is called a zonal spherical function.

Set

$$
\mathbb{T}_{1}:=\exp \left(i \mathfrak{a}_{\mathbb{R}}\right) /\left(\exp \left(i \mathfrak{a}_{\mathbb{R}}\right) \cap K^{\prime}\right) \simeq i \mathfrak{a}_{\mathbb{R}} / 2 \pi i Q_{\Sigma}^{\vee}
$$

with $Q_{\Sigma}^{\vee} \subset \mathfrak{a}_{\mathbb{R}}$ the coroot lattice of $\Sigma$. More explicitly, the coroot lattice $Q_{\Sigma}^{\vee}$ is the 
$\mathbb{Z}$-span of the elements $\frac{1}{2}\left(h_{i}-h_{i^{\prime}}\right)(1 \leq i \leq l), \exp \left(i \mathfrak{a}_{\mathbb{R}}\right)$ are the diagonal matrices

$$
\operatorname{diag}\left(e^{i \theta_{1}}, \ldots e^{i \theta_{l}}, 1, \ldots, 1, e^{-i \theta_{l}}, \ldots, e^{-i \theta_{1}}\right) \quad\left(\theta_{j} \in[0,2 \pi)\right),
$$

and $\exp \left(i \mathfrak{a}_{\mathbb{R}}\right) \cap K^{\prime}$ are the matrices in $\exp \left(i \mathfrak{a}_{\mathbb{R}}\right)$ of order 2 .

Write $\log : \mathbb{T}_{l} \rightarrow i \mathfrak{a}_{\mathbb{R}}$ for the multi-valued inverse of the exponential map exp : $i \mathfrak{a}_{\mathbb{R}} \rightarrow \mathbb{T}_{l}$. Similarly as for $A(\mathbb{T})$, the algebra of polynomial functions on $\mathbb{T}_{l}$ may be identified with the algebra $\mathbb{C}\left[x^{ \pm 1}\right]$ of Laurent polynomials in the $l$ variables $x_{j}$ $(1 \leq j \leq l)$, where the identification is given by $x_{j}(t):=e^{\varepsilon_{j}(\log (t))}$. In other words, the map $x_{j}$ is given by

$$
x_{j}: \operatorname{diag}\left(e^{i \theta_{1}}, \ldots e^{i \theta_{l}}, 1, \ldots, 1, e^{-i \theta_{l}}, \ldots, e^{-i \theta_{1}}\right) \longmapsto e^{2 i \theta_{j}}
$$

If follows that the algebra $\mathbb{C}\left[x^{ \pm 1}\right]$ of polynomial functions on $\mathbb{T}_{l}$ can be naturally embedded in the algebra $\mathbb{C}\left[z^{ \pm 1}\right]$ of polynomial functions on the maximal torus $\mathbb{T}$ by the assignment

$$
x_{1}=z_{1} z_{n}^{-1}, \quad x_{2}=z_{2} z_{n-1}^{-1}, \quad \ldots, \quad x_{l}=z_{l} z_{n+1-l}^{-1} .
$$

Let $\mathfrak{S}:=\mathfrak{S}_{l}$ denote the permutation group on $l$ letters, $\mathscr{W}:=\mathscr{W}_{l}=\mathbb{Z}_{2}^{l} \rtimes \mathfrak{S}_{l}$ the Weyl group of $\Sigma$. The natural action of $\mathscr{W}$ on $\mathfrak{a}_{\mathbb{R}}$ descends to $\mathbb{T}_{l}$. Hence $\mathscr{W}$ acts naturally on the algebra $\mathbb{C}\left[x^{ \pm 1}\right]$. Write $\mathbb{C}\left[x^{ \pm 1}\right]^{\mathscr{W}}$ for the subalgebra of $\mathscr{W}$-invariant Laurent polynomials. By Chevalley's restriction theorem and the above-mentioned natural embedding of $\mathbb{C}\left[x^{ \pm 1}\right]$ into $\mathbb{C}\left[z^{ \pm 1}\right]$, we have the following theorem.

Theorem 2.2. Restriction to $\mathbb{T}$ induces an isomorphism of $\mathscr{H}$ onto the algebra $\mathbb{C}\left[x^{ \pm 1}\right]^{\mathscr{W}}$ of $\mathscr{W}$-invariant Laurent polynomials in the variables $x_{i}(1 \leq i \leq l)$.

By Theorem 2.2 the direct sum decomposition (2.8) of $\mathscr{H}$ gives rise to a unique (up to rescaling) linear basis of $\mathbb{C}\left[x^{ \pm 1}\right]^{\mathscr{W}}$. This linear basis can be expressed in terms of $B C$ type Heckman-Opdam polynomials, which we will now define.

Let $V_{H O}$ denote the set of triples $k:=\left(k_{1}, k_{2}, k_{3}\right)$ of real numbers such that $k_{1}$ $+k_{3}>-\frac{1}{2}, k_{2}>0, k_{3}>-\frac{1}{2}$. Define an inner product $\langle\cdot, \cdot\rangle_{H O}=\langle\cdot, \cdot\rangle_{H O}^{k}$ on $\mathbb{C}\left[x^{ \pm 1}\right]^{\mathscr{W}}$ by

$$
\langle P, Q\rangle_{H O}:=\int_{\mathbb{T}_{l}} P(t) \overline{Q(t)} \Delta_{H O}(t ; k) d t
$$

Here $d t$ denotes the normalized Haar measure on the torus $\mathbb{T}_{l}$. The continuous positive weight function $t \longmapsto \Delta_{H O}(t ; k)$ on $\mathbb{T}_{l}$ is defined by 


$$
\Delta_{H O}(t ; k):=\prod_{\alpha \in \Sigma}\left(e^{\frac{1}{2} i\langle\alpha, \log (t)\rangle}-e^{-\frac{1}{2} i\langle\alpha, \log (t)\rangle}\right)^{k_{\alpha}} .
$$

The multiplicity parameters $k_{\alpha}$ are by definition equal to $k_{i}(i=1,2,3)$, depending on whether $\alpha \in \Sigma$ is a short, medium, or long root. It $l=1$ there is no dependence on $k_{2}$.

Recall that the usual orbit sums

$$
\tilde{m}_{\lambda}(x):=\sum_{\mu \in \mathscr{W}} x^{\mu} \quad\left(\lambda \in P_{\Sigma}^{+}\right)
$$

form a linear basis for the algebra $\mathbb{C}\left[x^{ \pm 1}\right]^{\mathscr{W}}$. The Heckman-Opdam hypergeometric orthogonal polynomials associated with the root system $\Sigma=B C_{l}$ (cf. [HS, Part I ]) are the uniquely determined family $\left\{\boldsymbol{P}_{\lambda}^{H O} \mid \lambda \in \boldsymbol{P}_{\Sigma}^{+}\right\}$such that

$$
\text { (i ) } \quad \boldsymbol{P}_{\lambda}^{H O}=\tilde{m}_{\lambda}+\sum_{\mu<\lambda} c_{\lambda \mu} \tilde{m}_{\mu} \text {, (ii) }\left\langle\boldsymbol{P}_{\lambda}^{H O}, \tilde{m}_{\mu}\right\rangle_{H O}=0 \text { for } \mu<\lambda \text {. }
$$

It is proved in [HS, p. 18] that, for any $k \in V_{H O}$, the $P_{\lambda}^{H O}(x ; k)$ are mutually orthogonal with respect to the inner product (2.11).

In the following theorem the zonal spherical functions on the symmetric space $U / K$ are expressed as $B C$ type Heckman-Opdam polynomials (cf. [HS, p. 76]).

Theorem 2.3. Under restriction to the maximal torus $\mathbb{T}$, the zonal $K^{\prime}$-spherical function $\varphi(\lambda)\left(\lambda \in P_{K}^{+}\right)$is mapped onto (a scalar multiple of) the Heckman-Opdam hypergeometric polynomial $P_{\lambda^{\natural O}}^{H O}(x ; k)$ with $k_{i}=\frac{1}{2} m_{i}(i=1,2,3)$.

Of course, the zonal $K$-spherical functions can be described in the same way, since the subgroups $K$ and $K^{\prime}$ are conjugate.

For later purposes, it is convenient to rewrite the zonal spherical functions in terms of generalized Jacobi polynomials, which are defined as follows. Write $\mathbb{C}[x]^{\mathcal{S}}$ for the algebra of symmetric polynomials in the variables $x_{i}(1 \leq i \leq l)$. A linear basis of this algebra is formed by the monomial symmetric polynomials $m_{\lambda}(x):=\sum_{\mu \in \subseteq_{\lambda}} x^{\mu}\left(\lambda \in P_{\Sigma}^{+}\right)$. Let $V_{J}$ denote the set of triples $(\alpha, \beta, \tau)$ of real numbers such that $\alpha, \beta>-1$ and $\tau>0$. For any $(\alpha, \beta, \tau) \in V_{J}$, we define an inner product $\langle\cdot, \cdot\rangle_{J}=\langle\cdot, \cdot\rangle_{J}^{\alpha, \beta, \tau}$ on $\mathbb{C}[x]^{\mathcal{S}}$ by

$$
\langle P, Q\rangle_{J}:=\int_{x_{1}=0}^{1} \cdots \int_{x_{l}=0}^{1} P(x) \overline{Q(x)} \Delta_{J}(x ; \alpha, \beta ; \tau) d x,
$$

with $d x=d x_{1} \ldots d x_{n}$ and

$$
\Delta_{J}(x ; \alpha, \beta ; \tau):=\prod_{i=1}^{l}\left(1-x_{i}\right)^{\beta} x_{i}^{\alpha}|\Delta(x)|^{2 \tau},
$$


where $\Delta(x):=\prod_{i<j}\left(x_{i}-x_{j}\right)$ is the Vandermonde determinant. The generalized Jacobi polynomials $\left\{\boldsymbol{P}_{\lambda}^{J}(\boldsymbol{x} ; \alpha, \beta ; \tau) \mid \lambda \in \boldsymbol{P}_{\Sigma}^{+}\right\}$(cf. Vretare $[\mathrm{Vr}]$ ) can now be defined by the same conditions (2.14) with $\tilde{m}_{\lambda}$ replaced by $m_{\lambda}$ and $\langle\cdot, \cdot\rangle_{\text {Ho }}$ replaced by $\langle\cdot, \cdot\rangle_{J}$.

As shown in $[\mathrm{SK}, \S 3]$, the simple change of variables $\boldsymbol{x}_{i} \longmapsto-\frac{1}{4}\left(\boldsymbol{x}_{i}+\boldsymbol{x}_{i}^{-1}-\right.$ 2) transforms the symmetric polynomials $P_{\lambda}^{J}$ into (a scalar multiple of) the $\mathscr{W}$ invariant Laurent polynomials $P_{\lambda}^{H O}$. The parameter correspondence is

$$
\alpha=k_{1}+k_{3}-\frac{1}{2}, \quad \beta=k_{3}-\frac{1}{2}, \quad \tau=k_{2} .
$$

Under this change of variables, the orthogonality relations of the Heckman-Opdam polynomials with respect to $\langle\cdot, \cdot\rangle_{H O}$ become the orthogonality relations of the generalized Jacobi polynomials $P_{\lambda}^{J}$ with respect to $\langle\cdot, \cdot\rangle_{J}$. The integral of the weight function $\Delta_{J}(x ; \alpha, \beta ; \tau)$ was first evaluated by Selberg in his well-known paper $[\mathrm{Sb}]$ :

$$
\begin{aligned}
\int_{x_{1}=0}^{1} \cdots \int_{x_{l}=0}^{1} & \prod_{l<j}\left|x_{i}-x_{j}\right|^{2 \tau} \prod_{l=1}^{l} x_{i}^{\alpha}\left(1-x_{i}\right)^{\beta} d x= \\
& \prod_{j=1}^{l} \frac{\Gamma(\alpha+1+(j-1) \tau) \Gamma(\beta+1+(j-1) \tau) \Gamma(j \tau+1)}{\Gamma(\alpha+\beta+2+(l+j-2) \tau) \Gamma(\tau+1)} .
\end{aligned}
$$

By the above-described relation between $B C$ type Heckman-Opdam polynomials and generalized Jacobi polynomials, the zonal spherical functions in Theorem 2.3 can be rewritten as generalized Jacobi polynomials with parameter values $\alpha=n-$ $2 l, \beta=0$ and $\tau=1$.

Observe that the zonal spherical functions, being matrix coefficients of irreducible representations, are mutually orthogonal with respect to the $L^{2}$ inner product on $A \subset L^{2}(U, d g$ ), where $d g$ is the normalized Haar measure on $U$ (Schur orthogonality). The restriction of the $L^{2}$ inner product to the algebra $\mathscr{H}$ of bi- $K^{\prime}-$ invariant matrix coefficients coincides under the isomorphism of Theorem 2.2 with the inner product $\langle\cdot, \cdot\rangle_{H O}$ on $\mathbb{C}\left[x^{ \pm 1}\right]^{\mathscr{W}}$ up to a non-zero positive constant. This constant can be explicitly determined using the evaluation of the Selberg integral (2.17).

\section{§3. BC Type $q$-Hypergeometric Orthogonal Polynomials}

In this section we recall three families of multivariable ( $B C$ type) basic hypergeometric orthogonal polynomials. Later on, certain subfamilies of these multivariable orthogonal polynomials will be interpreted as zonal spherical functions on quantizations of the classical complex Grassmannian. These families are $q$-analogues of the generalized Jacobi polynomials (or, equivalently, $q$ - 
analogues of the $B C$ type Heckman-Opdam polynomials) and multivariable analogues of well-known families of basic hypergeometric orthogonal polynomials occurring in the Askey tableau [AW].

We start with introducing some notations and conventions. We fix $0<q<1$ in the remainder of this paper. The $q$-shifted factorial is given by

$$
(a ; q)_{i}:=\prod_{j=0}^{t-1}\left(1-q^{j} a\right) \quad\left(i \in \mathbb{Z}_{+}\right), \quad(a ; q)_{\infty}:=\prod_{j=0}^{\infty}\left(1-q^{j} a\right) .
$$

We use the shorthand notation

$$
\left(a_{1}, \ldots, a_{m} ; q\right)_{i}:=\prod_{j=1}^{m}\left(a_{j} ; q\right)_{i} \quad\left(i \in \mathbb{Z}_{+} \cup\{\infty\}\right)
$$

for products of $q$-shifted factorials. Fix $N \in \mathbb{Z}$ and $\alpha, \beta \in \mathbb{C}$. The Jackson $q$-integral truncated at $N$ is defined by

$$
\begin{aligned}
& \int_{\alpha}^{\beta} f(x) d_{q, N} x:=\int_{0}^{\beta} f(x) d_{q, N} x-\int_{0}^{\alpha} f(x) d_{q, N} x, \\
& \int_{0}^{\beta} f(x) d_{q, N} x:=\sum_{k=0}^{N} f\left(\beta q^{k}\right)\left(\beta q^{k}-\beta q^{k+1}\right) \text { if } \quad N \geq 0, \\
& \int_{\alpha}^{\beta} f(x) d_{q, N} x:=0 \quad \text { if } \quad N<0 .
\end{aligned}
$$

The (non-truncated) Jackson $q$-integral is defined by

$$
\int_{\alpha}^{\beta} f(x) d_{q} x:=\lim _{N \rightarrow \infty} \int_{\alpha}^{\beta} f(x) d_{q, N} x,
$$

provided the limit exists. The three families of multivariable orthogonal polynomials we recall in this section are the Koornwinder polynomials (cf. [K3], [S3], [S4]), and the multivariable big and little $q$-Jacobi polynomials (cf. [S1], [S4]). The Koornwinder polynomials are multivariable analogues of the well-known Askey-Wilson polynomials. In the multivariable setting, these families depend on one additional deformation parameter $t \in(0,1)$. Since we only need the case $t=$ $q^{k}$ with $k \geq 1$ integer in this paper, we restrict ourselves to recalling the definitions and results for this special case. We first specify the parameter domain $V_{X}$ and the orthogonality inner product $\langle\cdot, \cdot\rangle_{X}$ for each family $(X=K, B, L)$, where $K$ stands for Koornwinder polynomials, and $B$ resp. $L$ stands for big resp. little $q$-Jacobi polynomials.

For the case $X=K$ we refer to $[\mathrm{K} 3],[\mathrm{S} 3],[\mathrm{S} 4]$. Take $V_{K}$ to be the set of quadruples $\underline{t}:=\left(t_{0}, t_{1}, t_{2}, t_{3}\right)$ such that

(1) $t_{0}, t_{1}, t_{2}, t_{3}$ are real, or appear in complex conjugate pairs,

(2) $t_{i} t_{j} \notin \mathbb{R}_{\geq 1}$ for $0 \leq i<j \leq 3$.

Fix $\underline{t} \in V_{K}$. For $e \in\left\{t_{0}, t_{1}, t_{2}, t_{3}\right\}$ with $|e|>1$, let $N_{e} \in \mathbb{Z}$ be the largest integer such that $\left|e q^{N_{e}}\right|>1$. Take $N_{e}:=-1$ if $|e| \leq 1$. Let $\int_{\mathbb{T}_{k}} d x_{1} \cdots d x_{k}$ denote $k$-fold contour 
integration along the complex unit circle in counterclockwise direction. For $0 \leq m$ $\leq l$ define a sesquilinear form $\langle\cdot, \cdot\rangle_{m, q, t}^{t}$ on the space $\mathbb{C}\left[x^{ \pm 1}\right]^{\mathscr{W}}$ by

$$
\begin{aligned}
&\langle P, Q\rangle_{m}:= \frac{2^{m}\left(\begin{array}{l}
l \\
m
\end{array}\right)}{(2 \pi i)^{l-m}} \sum_{e_{1}, \ldots, e_{m}} \int_{x_{1}=0}^{e_{1}} \cdots \int_{x_{m}=0}^{e_{m}} \int \cdots \int_{\left(x_{m+1}, \ldots, x_{l}\right) \in \mathbb{T}_{l-m}} \\
& P(x) \overline{Q(x)} \Delta_{m}(x) \frac{d_{q, N_{e}} x_{1}}{(1-q) x_{1}} \cdots \frac{d_{q, N_{e_{m}}} x_{m}}{(1-q) x_{m}} \frac{d x_{m+1}}{x_{m+1}} \cdots \frac{d x_{l}}{x_{l}} .
\end{aligned}
$$

Here the sum is taken over all $e_{j} \in\left\{t_{0}, t_{1}, t_{2}, t_{3}\right\}$, and the partial weight function $\Delta_{m}\left(x ; \underline{t} ; q, q^{k}\right)$ is defined by

$$
\Delta_{m}(x):=\prod_{r=1}^{m} w_{1}\left(x_{r} ; e_{r} ; f_{r}, g_{r}, h_{r} ; q\right) \prod_{s=m+1}^{l} w_{2}\left(x_{s} ; \underline{t} ; q\right) \prod_{\substack{c<j \\ \varepsilon, \varepsilon^{\prime} \in\{ \pm 1\}}}\left(x_{i}^{\varepsilon} x_{j}^{\varepsilon^{\prime}} ; q\right)_{k},
$$

with $w_{2}$ the continuous weight function of the one-variable Askey-Wilson polynomials,

$$
w_{2}(x ; \underline{t} ; q):=\frac{\left(x^{2}, x^{-2} ; q\right)_{\infty}}{\left(t_{0} x, t_{0} / x, t_{1} x, t_{1} / x, t_{2} x, t_{2} / x, t_{3} x, t_{3} / x ; q\right)_{\infty}},
$$

and

$$
w_{1}\left(e q^{i} ; e ; f, g, h ; q\right):=\operatorname{res}_{x=e q^{i}}\left(\frac{w_{1}(x ; \underline{t} ; q)}{x}\right),
$$

where the $f, g, h$ are such that $(e, f, g, h)$ is a permutation of $\left(t_{0}, t_{1}, t_{2}, t_{3}\right)$. Then

$$
\langle P, Q\rangle_{K}:=\sum_{m=0}^{n}\langle P, Q\rangle_{m} \quad\left(P, Q \in \mathbb{C}\left[x^{ \pm 1}\right]^{\mathscr{W}}\right)
$$

defines a positive definite inner product $\langle\cdot ; \cdot\rangle_{K}=\langle\cdot, \cdot\rangle_{K, q, t}^{t}$, which is symmetric in the parameters $t_{0}, t_{1}, t_{2}, t_{3}$.

The integral of the weight function $\mathscr{I}_{K, q, t}^{t}:=\langle 1,1\rangle_{K, q, t}^{t}$ is a $q$-analogue of Selberg's integral (2.17):

$$
\mathscr{I}_{K, q, t}^{t}=2^{l} l ! \prod_{j=1}^{l} \frac{\left(t, t^{l+j-2} t_{0} t_{1} t_{2} t_{3} ; q\right)_{\infty}}{\left(t^{j}, q, t_{0} t_{1} t^{j-1}, t_{0} t_{2} t^{j-1}, t_{0} t_{3} t^{j-1}, t_{1} t_{2} t^{j-1}, t_{1} t_{3} t^{j-1}, t_{2} t_{3} t^{j-1} ; q\right)_{\infty}} .
$$

For the case $\left|t_{0}\right|,\left|t_{1}\right|,\left|t_{2}\right|,\left|t_{3}\right|<1$ this was first proved by Gustafson [G]. The general case was proved in $[\mathrm{S} 4]$.

For the multivariable big and little $q$-Jacobi polynomials $(X=B, L)$ we refer to $[\mathrm{S} 1]$ and $[\mathrm{S} 4]$. Take $V_{B}$ to be the set of quadruples $(a, b, c, d)$ such that $c, d>0$ and 


$$
a \in(-c / d q, 1 / q), b \in(-d / c q, 1 / q)
$$

or $a=c z, b=-d \bar{z}$ with $z \in \mathbb{C} \backslash \mathbb{R}$. Fix $(a, b, c, d) \in V_{B}$ and define a positive definite inner product $\langle\cdot, \cdot\rangle_{B}=\langle\cdot, \cdot\rangle_{B, q, t}^{a, b, c, d}$ on $\mathbb{C}[x]^{\mathbb{S}}$ by

$$
\langle P, Q\rangle_{B}=\int_{x_{1}=-d}^{c} \int_{x_{2}=-d}^{c} \cdots \int_{x_{l}=-d}^{c} P(x) \overline{Q(x)} \Delta_{B}(x) d_{q} x_{1} \ldots d_{q} x_{l},
$$

with weight function $\Delta_{B}\left(x ; a, b, c, d ; q, q^{k}\right)$ given by

$$
\Delta_{B}(x)=\Delta(x) \prod_{i=1}^{l} w_{B}\left(x_{i}\right) \prod_{i<j} x_{i}^{2 k-1}\left(q^{1-k} x_{j} / x_{i} ; q\right)_{2 k-1} .
$$

Here $\Delta(x):=\prod_{1 \leq i<j \leq l}\left(x_{i}-x_{j}\right)$ is the Vandermonde determinant and

$$
w_{B}(x ; a, b, c, d ; q):=\frac{(q x / c,-q x / d ; q)_{\infty}}{(q a x / c,-q b x / d ; q)_{\infty}}
$$

is the weight function of the one-variable big $q$-Jacobi polynomials. The evaluation of the integral $\mathscr{I}_{B, q, t}^{a, b, c, d}:=\langle 1,1\rangle_{B, q, t}^{a, b, c, d}$ was first conjectured by Askey [A] and subsequently proved by Evans [E]:

$$
\begin{aligned}
& \mathscr{I}_{B, q, i}^{a, b, c, d}=l ! q^{k^{2}\left(\begin{array}{l}
l \\
3
\end{array}\right)-\left(\begin{array}{c}
k \\
2
\end{array}\right)\left(\begin{array}{l}
l \\
2
\end{array}\right)} \prod_{i=1}^{l} \frac{\Gamma_{q}(\alpha+1+(i-1) k) \Gamma_{q}(\beta+1+(i-1) k) \Gamma_{q}(i k)}{\Gamma_{q}(\alpha+\beta+2+(l+i-2) k) \Gamma_{q}(k)} \\
& \times \prod_{i=1}^{l} \frac{(-d / c,-c / d ; q)_{\infty}(c d)^{1+(i-1) k}}{\left(-q^{\alpha+1+(i-1) k} d / c,-q^{\beta+1+(i-1) k} c / d ; q\right)_{\infty}(c+d)},
\end{aligned}
$$

where $t=q^{k}$ ( $k \geq 1$ integer), $a=q^{\alpha}, b=q^{\beta}$ and with

$$
\Gamma_{q}(a):=(1-q)^{1-a} \frac{(q ; q)_{\infty}}{\left(q^{a} ; q\right)_{\infty}} \quad\left(a \notin \neq-\mathbb{Z}_{+}\right)
$$

the $q$-Gamma function. Note that (3.6) is another $q$-analogue of Selberg's integral (2.17).

Finally, take $V_{L}$ to be the set of pairs $(a, b)$ such that $a \in(0,1 / q)$ and $b \in$ $(-\infty, 1 / q)$. Fix $(a, b) \in V_{L}$ and define a positive definite inner product $\langle\cdot, \cdot\rangle_{L}=$ $\langle\cdot, \cdot\rangle_{L, q, t}^{a, b}$ on $\mathbb{C}[x]^{\mathcal{S}}$ by

$$
\langle P, Q\rangle_{L}=\int_{x_{1}=0}^{1} \cdots \int_{x_{l}=0}^{1} P(x) \overline{Q(x)} \Delta_{L}(x) d_{q} x_{1} \ldots d_{q} x_{l},
$$

with weight function $\Delta_{L}\left(x ; a, b ; q, q^{k}\right)$ given by

$$
\Delta_{L}(x)=\Delta(x) \prod_{i=1}^{l} w_{L}\left(x_{i}\right) \prod_{i<j} x_{i}^{2 k-1}\left(q^{1-k} x_{j} / x_{i} ; q\right)_{2 k-1},
$$

and 


$$
w_{L}(x ; a, b ; q):=\frac{(q x ; q)_{\infty}}{(q b x ; q)_{\infty}} x^{\alpha} \quad\left(a=q^{\alpha}\right)
$$

the weight function of the one-variable little $q$-Jacobi polynomials. The evaluation of the integral $\mathscr{I}_{L, q, t}^{a, b}:=\langle 1,1\rangle_{L, q, t}^{a, b}$ was conjectured by Askey [A] and proved independently by Habsieger $[\mathrm{Ha}]$ and $\mathrm{Kadell}[\mathrm{Ka}]$ :

$$
\begin{aligned}
\mathscr{I}_{L, q, t}^{a, b}= & l ! q^{k(\alpha+1)\left(\begin{array}{l}
l \\
2
\end{array}\right)+2 k^{2}\left(\begin{array}{l}
l \\
3
\end{array}\right)} \\
& \times \prod_{i=1}^{l} \frac{\Gamma_{q}(\alpha+1+(i-1) k) \Gamma_{q}(\beta+1+(i-1) k) \Gamma_{q}(i k)}{\Gamma_{q}(\alpha+\beta+2+(l+i-2) k) \Gamma_{q}(k)},
\end{aligned}
$$

where $t=q^{k}$ ( $k \geq 1$ integer), $a=q^{\alpha}, b=q^{\beta}$. This integral is yet another $q$-analogue of Selberg's integral (2.17).

We now define the corresponding families of orthogonal polynomials.

It will be convenient to write $m_{\lambda}^{X}\left(\lambda \in P_{\Sigma}^{+}\right)$for $\tilde{m}_{\lambda}(X=K)$ or $m_{\lambda}(X=B, L)$. Fix $t=q^{k} \quad(k \geq 1$ integer $)$ and fix parameters in $V_{X}$. The Koornwinder, multivariable big resp. little $q$-Jacobi polynomials $\left\{P_{\lambda}^{X} \mid \lambda \in P_{\Sigma}^{+}\right\}(X=K, B$ resp. $L)$ are uniquely determined by the following two conditions :

$$
\text { （i ） } P_{\lambda}^{X}=m_{\lambda}^{X}+\sum_{\mu<\lambda} c_{\lambda \mu}^{X} m_{\mu}^{X} \text {, (ii ) }\left\langle P_{\lambda}^{X}, m_{\mu}^{X}\right\rangle_{X}=0 \text { for } \mu<\lambda \text {. }
$$

After suitable rescaling of the parameters, the Koornwinder polynomials tend to the Heckman-Opdam hypergeometric polynomials associated with $B C_{l}$ in the limit $q \rightarrow 1$ (cf. $[\mathrm{Dj} 1, \S 4]$ ), and the multivariable big resp. little $q$-Jacobi polynomials tend to the generalized Jacobi polynomials in the limit $q \rightarrow 1$ (cf. [SK, Thm. 5.1]).

The $P_{\lambda}^{X}$ are joint eigenfunctions of a certain second-order $q$-difference operator $D_{X}$ which is self-adjoint with respect to $\langle\cdot, \cdot\rangle_{X}$ :

$$
D_{X}:=\sum_{j=1}^{n} \phi_{X, j}^{+}(x)\left(T_{q, j}-\mathrm{Id}\right)+\phi_{X, j}^{-}(x)\left(T_{q}^{-1, j}-\mathrm{Id}\right) \quad(X=K, B, L),
$$

where $T_{q} \pm 1, j$ is the (multiplicative) $q^{ \pm 1}$-shift in the variable $x_{j}$. In the case $X=K$ one has explicitly

$$
\begin{aligned}
\phi_{K, j}^{+}(x) & :=\frac{\left(1-t_{0} x_{j}\right)\left(1-t_{1} x_{j}\right)\left(1-t_{2} x_{j}\right)\left(1-t_{3} x_{j}\right)}{\left(1-x_{j}^{2}\right)\left(1-q x_{j}^{2}\right)} \prod_{i \neq j} \frac{\left(1-t x_{i} x_{j}\right)\left(1-t x_{i}^{-1} x_{j}\right)}{\left(1-x_{i} x_{j}\right)\left(1-x_{i}^{-1} x_{j}\right)}, \\
\phi_{K, j}^{-}(x) & :=\frac{\left(t_{0}-x_{j}\right)\left(t_{1}-x_{j}\right)\left(t_{2}-x_{j}\right)\left(t_{3}-x_{j}\right)}{\left(1-x_{j}^{2}\right)\left(q-x_{j}^{2}\right)} \prod_{l \neq j} \frac{\left(t-x_{i} x_{j}\right)\left(t-x_{i}^{-1} x_{j}\right)}{\left(1-x_{i} x_{j}\right)\left(1-x_{i}^{-1} x_{j}\right)} .
\end{aligned}
$$

The eigenvalues in this case are given by 


$$
E_{\lambda}^{K}(\underline{t} ; q, t)=\sum_{j=1}^{l}\left(q^{-1} t_{0} t_{1} t_{2} t_{3} t^{2 l-j-1}\left(q^{\lambda_{j}}-1\right)+t^{j-1}\left(q^{-\lambda_{j}-1}\right)\right) .
$$

For the $q$-difference operators $D_{X}$ and their eigenvalues in the cases $X=B, L$ see [S1].

As shown in $[\mathrm{S} 3](X=K)$ and $[\mathrm{S} 1](X=B, L)$, the self-adjointness of $D_{X}$ with respect to $\langle\cdot, \cdot\rangle_{X}$ may be used to prove full orthogonality :

$$
\left\langle P_{\lambda}^{X}, P_{\mu}^{X}\right\rangle_{X}=0 \quad\left(\lambda, \mu \in P_{\Sigma}^{+}, \lambda \neq \mu\right)
$$

We remark that for $\underline{t} \in V_{K}$ with $\left|t_{0}\right|,\left|t_{1}\right|,\left|t_{2}\right|,\left|t_{3}\right|<1$, the orthogonality measure for the Koornwinder polynomials reduces to the completely continuous orthogonality measure which was considered for the first time by Koornwinder [K3].

For certain values of the parameters, for instance $t_{0} t_{1} t_{2} t_{3} \in[-q, 1)$, one has $E_{\lambda}^{K} \neq E_{\mu}^{K}$ if $\lambda<\mu$ (cf. [SK, Prop. 4.6]). The Koornwinder polynomials may then be characterized by the conditions

$$
\text { (i ) } P_{\lambda}^{K}=\tilde{m}_{\lambda}+\sum_{\mu<\lambda} c_{\lambda \mu} \tilde{m}_{\mu} \text {, (ii) } D_{K} P_{\lambda}^{K}=E_{\lambda}^{K} P_{\lambda}^{K}
$$

Using this characterization, we can read off certain elementary symmetry properties of the Koornwinder polynomials from the corresponding symmetry properties of Koornwinder's second-order $q$-difference operator and its eigenvalues. In the following lemma we formulate two of them.

Lemma 3.1. Let $\underline{t} \in V_{K}$ such that $t_{0} t_{1} t_{2} t_{3} \in[-q, 1)$. Then the Koornwinder polynomial $P_{\lambda}(. ; \underline{t} ; q, t)$ is symmetric in the four parameters $t_{0}, t_{1}, t_{2}$ and $t_{3}$, and satisfies

$$
P_{\lambda}(x ;-\underline{t} ; q, t)=(-1)^{|\lambda|} P_{\lambda}(-x ; \underline{t} ; q, t),
$$

where $-x:=\left(-x_{1}, \ldots,-x_{l}\right)$ and, similarly, $-\underline{t}=\left(-t_{0},-t_{1},-t_{2},-t_{3}\right)$.

Write $|\lambda|:=\sum_{i=1}^{n} \lambda_{i}$ for $\lambda \in P_{\Sigma}^{+}$, and $c x:=\left(c x_{1}, \ldots, c x_{l}\right)$ for $c \in \mathbb{C}$. We have the following limit transitions from Koornwinder polynomials to multivariable big resp. little $q$-Jacobi polynomials (cf. $[\mathrm{SK}],[\mathrm{S} 2]$ ). Fix $\lambda \in P_{\Sigma}^{+}$and $k \geq 1$. For $(a, b, c, d) \in V_{B}$ we have the limit transition

$$
\lim _{\varepsilon \downarrow 0}\left(\frac{\varepsilon(c d)^{\frac{1}{2}}}{q^{\frac{1}{2}}}\right)^{|\lambda|} P_{\lambda}^{K}\left(\frac{q^{\frac{1}{2}} x}{\varepsilon(c d)^{\frac{1}{2}}} ; \underline{t}_{B}(\varepsilon) ; q, q^{k}\right)=P_{\lambda}^{B}\left(x ; a, b, c, d ; q, q^{k}\right),
$$

where 


$$
\underline{t}_{B}(\varepsilon):=\left(\varepsilon^{-1}(q c / d)^{\frac{1}{2}},-\varepsilon^{-1}(q d / c)^{\frac{1}{2}}, \varepsilon a(q d / c)^{\frac{1}{2}},-\varepsilon b(q c / d)^{\frac{1}{2}}\right) .
$$

For $(a, b) \in V_{L}$ we have the limit transition

$$
\lim _{\varepsilon \downarrow 0}\left(\frac{\varepsilon}{q^{\frac{1}{2}}}\right){ }^{|\lambda|} P_{\lambda}^{K}\left(\frac{q^{\frac{1}{2}} x}{\varepsilon} ; \underline{t}_{L}(\varepsilon) ; q, q^{k}\right)=P_{\lambda}^{L}\left(x ; a, b ; q, q^{k}\right),
$$

where

$$
\underline{t}_{L}(\varepsilon):=\left(\varepsilon^{-1} q^{\frac{1}{2}},-a q^{\frac{1}{2}}, \varepsilon b q^{\frac{1}{2}},-q^{\frac{1}{2}}\right) .
$$

To understand how $\mathscr{W}$-invariant Laurent polynomials can tend to symmetric polynomials observe that $\lim _{\varepsilon \downarrow 0} \varepsilon^{|\lambda|} \tilde{m}_{\lambda}(x / \varepsilon)=m_{\lambda}(x)\left(\lambda \in P_{\Sigma}^{+}\right)$.

Let us write $m_{i}^{X}(1 \leq i \leq l)$ for the orbit sum in $\mathbb{C}\left[x^{ \pm 1}\right]^{\mathscr{W}}$ corresponding to the $i$-th fundamental weight $(X=K)$ resp. for the $i$-th elementary symmetric polynomial in $\mathbb{C}[x]^{\mathcal{S}}(X=B, L)$. It is known (see [B, Ch. VI, $\S 4$, Thm. 1]) that the $m_{i}^{X}$ $(1 \leqq i \leq l)$ are algebraically independent and generate the algebra $\mathbb{C}\left[x^{ \pm 1}\right]^{\mathscr{W}}$ resp. $\mathbb{C}[x]^{\oplus}$. It therefore makes sense to introduce the notation

$$
\hat{P}_{\lambda}^{X}\left(m_{1}^{X}(x), \ldots, m_{l}^{X}(x)\right):=P_{\lambda}^{X}(x) \quad\left(\lambda \in P_{\Sigma}^{+}\right)
$$

The $\hat{P}_{\lambda}^{X}$ are (non-symmetric) polynomials in $l$ variables. We may reformulate the above limit transitions in terms of these polynomials in the following way.

$$
\lim _{\varepsilon \downarrow 0}\left(s_{\varepsilon}^{-1}\right)^{|\lambda|} \hat{P}_{\lambda}^{K}\left(s_{\varepsilon} y_{1}, \ldots, s_{\varepsilon}^{l} y_{l} ; \underline{t}_{B}(\varepsilon) ; q, q^{k}\right)=\hat{P}_{\lambda}^{B}\left(y_{1}, \ldots, y_{l} ; a, b, c, d ; q, q^{k}\right)
$$

with $s_{\varepsilon}:=q^{\frac{1}{2}} / \varepsilon(c d)^{\frac{1}{2}}$, and

$$
\lim _{\varepsilon \downarrow 0}\left(s_{\varepsilon}^{-1}\right)^{|\lambda|} \hat{P}_{\lambda}^{K}\left(s_{\varepsilon} y_{1}, \ldots, s_{\varepsilon}^{l} y_{l} ; \underline{t}_{L}(\varepsilon) ; q, q^{k}\right)=\hat{P}_{\lambda}^{L}\left(y_{1}, \ldots, y_{l} ; a, b ; q, q^{k}\right)
$$

with $s_{\varepsilon}:=q^{\frac{1}{2}} / \varepsilon$.

Important for our applications of the multivariable orthogonal polynomial theory to the study of zonal spherical functions on quantum Grassmannians is the fact that the limit transitions (3.14) and (3.16) extend to rigorous limits on the level of the orthogonality measure. This means that the orthogonality measure of the Koornwinder polynomials tends (in a suitable weak sense) to a non-zero multiple of the orthogonality measure of the multivariable big respectively little $q$ Jacobi polynomials in the limit (3.14) resp. (3.16) (see [S4]). In particular, the quadratic norms of the multivariable big and little $q$-Jacobi polynomials can be derived by computing the limits (3.14) and (3.16) in the quadratic norm expressions of the Koornwinder polynomials (cf. [S4]). 
We reformulate this observation as follows. For parameters $\underline{t} \in V_{K},(a, b, c, d)$ $\in V_{B}$ and $(a, b) \in V_{L}$, let the renormalized quadratic norms $N_{K}(\lambda):=N_{K}(\lambda ; \underline{t} ; q$, $t), N_{B}(\lambda):=N_{B}(\lambda ; a, b, c, d ; q, t)$ and $N_{L}(\lambda):=N_{L}(\lambda ; a, b ; q, t)$ for $\lambda \in P_{\Sigma}^{+}$be defined by

$$
N_{X}(\lambda):=\frac{\left\langle P_{\lambda}^{X}, P_{\lambda}^{X}\right\rangle_{X}}{\langle 1,1\rangle_{X}}, \quad(X=K, B, L) .
$$

By the positive-definiteness of $\langle\cdot, \cdot\rangle_{X}$, it follows that $N_{X}\langle\lambda\rangle$ is strictly positive for all $\lambda \in P_{\Sigma}^{+}$.

Proposition 3.2. ([S4]) For $(a, b, c, d) \in V_{B}$, we have

$$
\lim _{\varepsilon \downarrow 0}\left(\varepsilon(c d / q)^{\frac{1}{2}}\right)^{2|\lambda|} N_{K}\left(\lambda ; t_{B}(\varepsilon) ; q, t\right)=N_{B}(\lambda ; a, b, c, d ; q, t), \quad\left(\lambda \in P_{\Sigma}^{+}\right) .
$$

For $(a, b) \in V_{L}$, we have

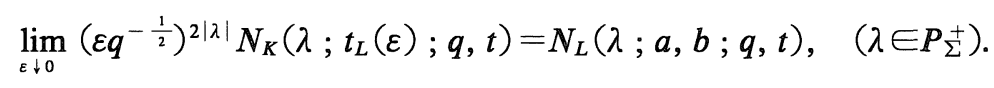

Remark 3.3. For the explicit evaluations of the quadratic norms of the Koornwinder polynomials and the multivariable big and little $q$-Jacobi polynomials in terms of products and quotients of $q$-Gamma functions, we refer the reader to [S4]. The quadratic norm evaluations for the Koornwinder polynomials were derived earlier in the paper $[\mathrm{Dj} 2]$.

\section{§4. Preliminaries on the Quantum Unitary Group}

Various aspects of the quantum unitary group have been studied in many different papers. Our main references will be $[\mathrm{NYM}]$ and $[\mathrm{N}, \S 1]$, which are based on the $R$-matrix approach described in [RTF].

The quantized coordinate ring $A_{q}(\operatorname{Mat}(n, \mathbb{C}))$ of the space of $n \times n$ complex matrices is defined as the algebra with generators $t_{i j}(1 \leq i, j \leq n)$ and relations

$$
\begin{array}{ll}
t_{k i} t_{k j}=q t_{k j} t_{k i}, & t_{i k} t_{j k}=q t_{j k} t_{i k} \quad(i<j), \\
t_{i l} t_{k j}=t_{k j} t_{i l}, & t_{i j} t_{k l}-t_{k l} t_{i j}=\left(q-q^{-1}\right) t_{i l} t_{k j} \quad(i<k, j<l) .
\end{array}
$$

In more compact notation, these relations may be written $R T_{1} T_{2}=T_{2} T_{1} R$. Here $T_{1}:=T \otimes I, T_{2}:=I \otimes T(I$ unit matrix, $\otimes$ the Kronecker product of matrices $)$, and

$$
R:=\sum_{i j} q^{\delta_{i j}} e_{i i} \otimes e_{i j}+\left(q-q^{-1}\right) \sum_{i>j} e_{i j} \otimes e_{j i}
$$


with the $e_{i j}(1 \leq i, j \leq n)$ denoting the standard matrix units. The matrix $R$ is invertible and satisfies the Quantum Yang-Baxter Equation.

The quantized coordinate $\operatorname{ring} A_{q}(G)$ of the general linear group $G=G L(n, \mathbb{C})$ is defined by adjoining to $A_{q}(\operatorname{Mat}(n, \mathbb{C}))$ the inverse $\operatorname{det}_{q}^{-1}$ of the quantum determinant

$$
\operatorname{det}_{q}:=\sum_{\sigma \in \mathbb{E}_{n}}(-q)^{l(\sigma)} t_{1 \sigma(1)} \ldots t_{n \sigma(n)} \in A_{q}\left(\operatorname{Mat}_{q}(n, \mathbb{C})\right)
$$

( $l$ denoting the length function on $\mathfrak{S}$ ), which is central. It follows from [NYM, Lemma 1.5$]$ that $A_{q}(\operatorname{Mat}(n ; \mathbb{C}))$ has no zero divisors.

There is a unique Hopf algebra structure on $A_{q}(G)$ such that $\left(t_{i j}\right)$ becomes a matrix corepresentation. The antipode $S: A_{q}(G) \rightarrow A_{q}(G)$ is given on the generators by

$$
S\left(t_{i j}\right):=(-q)^{i-j} \xi_{i^{c}}^{j^{c}} \operatorname{det}_{q}^{-1}
$$

with $i^{c}:=\{1, \ldots, n\} \backslash\{i\}$, and with the quantum minor $\xi_{J}^{I}$ for subsets $I=\left\{i_{1}<\cdots<\right.$ $\left.i_{r}\right\}, J=\left\{j_{1}<\cdots<j_{r}\right\} \subset\{1, \ldots, n\}$ defined by

$$
\xi_{J}^{I}:=\sum_{\sigma \in \mathbb{E}_{r}}(-q)^{l(\sigma)} t_{i_{1} j_{\sigma(1)}} \ldots t_{i_{r} j_{\sigma(r)}} .
$$

$A_{q}(G)$ becomes a Hopf $*$-algebra by requiring $\left(t_{i j}\right)$ to be a unitary matrix corepresentation. In particular, this means that the $*$-structure is given by $t_{i j}^{*}:=S\left(t_{j i}\right)$. We write $A_{q}(U)=A_{q}(U(n))$ for $A_{q}(G)$ endowed with this *-operation. The mapping $\tau:=* \circ S$ is a conjugate-linear involution on $A_{q}(U)$ such that $\tau\left(t_{i j}\right)=t_{j i}$.

The quantized Borel subgroups $A_{q}\left(B^{ \pm}\right)$of upper resp. lower triangular matrices are defined as the Hopf quotients of $A_{q}(G)$ by the relations

$$
t_{i j}=0 \quad(i>j) \quad \text { resp. } \quad t_{i j}=0 \quad(i<j) .
$$

The corresponding projections will be denoted by $\pi_{ \pm}: A_{q}(G) \rightarrow A_{q}\left(B^{ \pm}\right)$. Note that the $z_{i}:=\pi_{ \pm}\left(t_{i i}\right)(1 \leq i \leq n)$ in $A_{q}\left(B^{ \pm}\right)$are invertible. Corresponding to the diagonal subgroup $\mathbb{T} \subset U(n)$ we have a natural surjective Hopf $*$-algebra morphism ${ }_{\mid \mathbb{T}}$ of $A_{q}(U)$ onto the Laurent polynomial algebra $A(\mathbb{T}):=\mathbb{C}\left[z_{1}^{ \pm 1}, \ldots, z_{n}^{ \pm 1}\right]$.

Next, we briefly recall the "global" description of finite-dimensional corepresentations of $A_{q}(U)$. For every $\lambda \in P$ (cf. §2), we may define a linear character (i.e. one-dimensional corepresentation) $z^{\lambda}:=z_{1}^{\lambda_{1}} \cdots z_{n}^{\lambda_{n}}$ of $A_{q}\left(B^{ \pm}\right)$or $A(\mathbb{T})$. Using these linear characters it is completely straightforward to define (highest) weight vectors in left or right $A_{q}(U)$-comodules. We take highest weight vectors of right resp. left $A_{q}(U)$-comodules with respect to $A_{q}\left(B^{+}\right)$and $A_{q}\left(B^{-}\right)$respectively. For instance, a highest weight vector of weight $\lambda \in P^{+}$in a right $A_{q}(U)$-comodule $M$ is a non-zero vector $v \in M$ such that 


$$
\left(\mathrm{id} \otimes \pi_{+}\right) \circ \rho_{M}(v)=v \otimes z^{\lambda},
$$

$\rho_{M}: M \rightarrow M \otimes A_{q}(U)$ denoting the comodule mapping. Finite-dimensional $A_{q}(U)$ comodules are then completely reducible and unitarizable (see, for instance, $[\mathrm{NYM}],[\mathrm{DK} 2])$. Recall that a $\operatorname{right} A_{q}(U)$-comodule $M$ endowed with a positive definite inner product (taken to be conjugate linear in the second variable) is called unitary if

$$
\sum_{(v)(w)}\left\langle v_{(1)}, w_{(1)}\right\rangle w_{(2)}^{*} v_{(2)}=\langle v, w\rangle 1 \quad(v, w \in M),
$$

where the symbolic notation $\Sigma_{(v)} v_{(1)} \otimes v_{(2)}:=\rho_{M}(v)$ is used. The irreducible finitedimensional $A_{q}(U)$-comodules are parametrized by dominant weights $\lambda \in P^{+}$as in the classical case (cf. [NYM, Th. 2.12]). The irreducible right $A_{q}(U)$-comodule with highest weight $\lambda \in P^{+}$is denoted by $V_{R}(\lambda)$. The vector space $V_{L}(\lambda):=$ Hom $\left(V_{R}(\lambda), \mathbb{C}\right)$ has a natural left $A_{q}(U)$-comodule structure, which is also irreducible of highest weight $\lambda$. If no confusion is possible, we will write $V(\lambda)$ for the left comodule $V_{L}(\lambda)$ respectively for the right comodule $V_{R}(\lambda)$.

Remark 4.1. Let $M$ be a finite-dimensional right $A_{q}(U)$-comodule with comodule mapping $\rho_{M}: M \rightarrow M \otimes A_{q}(U)$. Write $M^{\circ}$ for the vector space complex conjugate to $M$ and $\sigma: M \otimes A_{q}(U) \rightarrow A_{q}(U) \otimes M$ for the flip. Then the mapping

$$
\rho_{M}^{\circ}: M^{\circ} \rightarrow A_{q}(U) \otimes M^{\circ}, \quad \rho_{m}^{\circ}:=(\tau \otimes \mathrm{id}) \circ \sigma \circ \rho_{M},
$$

where $\tau=* \circ S$, defines a left $A_{q}(U)$-comodule structure on $M^{\circ}$. In (4.2) $\rho_{M}$ is considered as a conjugate linear map from $M^{\circ}$ to $M \otimes A_{q}(U)$, and $\tau \otimes$ id as a conjugate linear map from $A_{q}(U) \otimes M$ to $A_{q}(U) \otimes M^{\circ}$.

The assignment $M \longmapsto M^{\circ}$ is a $1-1$ correspondence between right and left $A_{q}(U)$-comodules preserving weights and highest weights. Hence $M^{\circ}$ is isomorphic to the left $A_{q}(U)$-comodule Hom $(M, \mathbb{C})$. A right $A_{q}(U)$-comodule intertwiner $\Psi$ : $M \rightarrow N$ also intertwines the left $A_{q}(U)$-comodule structures of $M^{\circ}$ and $N^{\circ}$ (i.e. when $\Psi$ is considered as a map from $M^{\circ}$ to $N^{\circ}$ ).

Recall that the comultiplication $\Delta: A_{q}(U) \rightarrow A_{q}(U) \otimes A_{q}(U)$ defines a bicomodule structure on $A_{q}(U)$. Let $W(\lambda) \subset A_{q}(U)\left(\lambda \in P^{+}\right)$denote the subspace spanned by the matrix coefficients of either $V_{R}(\lambda)$ or $V_{L}(\lambda)$. The irreducible decomposition of the bicomodule $A_{q}(U)$ reads

$$
A_{q}(U)=\bigoplus_{\lambda \in P^{+}} W(\lambda), \quad W(\lambda) \simeq V_{L}(\lambda) \otimes V_{R}(\lambda)
$$

Let $h$ be the normalized Haar functional on $A_{q}(U)$. It can be characterized as the unique linear functional on $A_{q}(U)$ which is zero on $W(\lambda)$ for $0 \neq \lambda \in P^{+}$and which 
sends $1 \in A_{q}(U)$ to $1 \in \mathbb{C}$. The subspaces $W(\lambda)$ are mutually orthogonal with respect to the inner product $\langle\varphi, \phi\rangle:=h\left(\phi^{*} \varphi\right)$.

We consider now in some more detail the vector corepresentation, its dual representation, and their exterior powers. Let $V$ denote the vector space $\mathbb{C}^{n}$ with canonical basis $\left(v_{i}\right)$. $V$ becomes a right $A_{q}(U)$-comodule (called vector corepresentation) with

$$
\rho_{V}: V \longmapsto V \otimes A_{q}(U), \quad \rho_{V}\left(v_{j}\right):=\sum_{i=1}^{n} v_{i} \otimes t_{i j} \quad(1 \leq j \leq n) .
$$

$V$ is irreducible with highest weight $\tilde{\varepsilon}_{1}$ and highest weight vector $v_{1}$. Note that the vectors $v_{i}$ have weight $\tilde{\varepsilon}_{i}$. The corepresentation $V$ is unitary with respect to the inner product $\left\langle v_{i}, v_{j}\right\rangle=\delta_{i j}$.

Let $V^{*}$ denote the linear dual of $V$ with dual basis $\left(v_{i}^{*}\right) . V^{*}$ becomes a right $A_{q}(U)$-comodule (contragredient of $V$ ) with

$$
\rho_{V^{*}}: V^{*} \longmapsto V^{*} \otimes A_{q}(U), \quad \rho_{V^{*}}\left(v_{j}^{*}\right):=\sum_{i=1}^{n} v_{l}^{*} \otimes t_{i j}^{*} \quad(1 \leq j \leq n) .
$$

$V^{*}$ is irreducible with highest weight $-\tilde{\varepsilon}_{n}$ and highest weight vector $v_{n}^{*}$. Note that the vectors $v_{i}^{*}$ have weight $-\tilde{\varepsilon}_{i}$. The corepresentation $V^{*}$ is unitary with respect to the inner product $\left\langle v_{i}^{*}, v_{j}^{*}\right\rangle:=q^{-\left\langle 2 \rho, \tilde{\varepsilon}_{i}\right\rangle} \delta_{i j}$, where $\rho:=\sum_{k=1}^{n}(n-k) \tilde{\varepsilon}_{k}$. This follows from the well-known fact that $S^{2}\left(t_{i j}\right)=q^{\left\langle 2 \rho, \tilde{\varepsilon}_{j}-\tilde{\varepsilon}_{i}\right\rangle} t_{i j}(1 \leq i, j \leq n)$.

Let $\Lambda_{q}(V)$ resp. $\Lambda_{q}\left(V^{*}\right)$ denote the associative algebra generated by $v_{1}, \ldots, v_{n}$ resp. $v_{1}^{*}, \ldots, v_{n}^{*}$ with relations

$$
v_{i} \wedge v_{i}=0 \quad(1 \leq i \leq n), \quad v_{i} \wedge v_{j}=-q^{-1} v_{j} \wedge v_{i} \quad(i<j)
$$

respectively

$$
v_{i}^{*} \wedge v_{l}^{*}=0 \quad(1 \leq i \leq n), \quad v_{j}^{*} \wedge v_{i}^{*}=-q^{-1} v_{i}^{*} \wedge v_{j}^{*} \quad(i<j) .
$$

Then $\Lambda_{q}(V)$ resp. $\Lambda_{q}\left(V^{*}\right)$ inherits a natural right $A_{q}(U)$-comodule structure from $V$ resp. $V^{*}$ by extending the comodule mapping $\rho_{V}$ resp. $\rho_{V} *$ as a unital algebra homomorphism. $\Lambda_{q}(V)$ resp. $\Lambda_{q}\left(V^{*}\right)$ also has a natural grading such that the generators $v_{i}$ resp. $v_{i}^{*}$ have degree 1 :

$$
\Lambda_{q}(V)=\bigoplus_{r=0}^{n} \Lambda_{q}^{r}(V), \quad \Lambda_{q}\left(V^{*}\right)=\bigoplus_{r=0}^{n} \Lambda_{q}^{r}\left(V^{*}\right) .
$$

Write $v_{I}:=v_{i_{1}} \wedge \cdots \wedge v_{i_{r}}$ resp. $v_{I}^{*}:=v_{i_{r}}^{*} \wedge \cdots \wedge v_{i_{1}}^{*}$ if $I=\left\{i_{1}<\cdots<i_{r}\right\} \subset\{1, \ldots, n\}$. Write $|I|$ for the cardinality of $I$. Then the $v_{I}$ resp. $v_{I}^{*}(|I|=r)$ form a basis of $\Lambda_{q}^{r}(V)$ resp. $\Lambda_{q}^{r}\left(V^{*}\right)$. One has the multiplicative property $(I, J \subset\{1, \ldots, n\})$

$$
v_{I} \wedge v_{J}=\operatorname{sgn}_{q}(I ; J) v_{I \cup J}, \quad v_{I}^{*} \wedge v_{J}^{*}:=\operatorname{sgn}_{q}(J ; I) v_{I \cup J}^{*}
$$


where

$$
\operatorname{sgn}_{q}(I ; J):=\left\{\begin{array}{lll}
0 & \text { if } \quad I \cap J \neq \varnothing, \\
(-q)^{l(I ; J)} & \text { if } \quad I \cap J=\varnothing,
\end{array}\right.
$$

and $l(I ; J):=|\{(i, j) \in I \times J \mid i>j\}|$. The comodules $\Lambda_{q}^{r}(V)$ and $\Lambda_{q}^{r}\left(V^{*}\right)$ are irreducible subcomodules of $\Lambda_{q}(V)$, and the coactions satisfy

$$
\rho_{V}\left(v_{J}\right)=\sum_{|I|=r} v_{I} \otimes \xi_{J}^{I}, \quad \rho_{V} *\left(v_{J}^{*}\right)=\sum_{|I|=r} v_{I}^{*} \otimes\left(\xi_{J}^{I}\right)^{*} \quad(|J|=r) .
$$

From this it follows immediately that

$$
\Delta\left(\xi_{J}^{I}\right)=\sum_{|K|=r} \xi_{K}^{I} \otimes \xi_{J}^{K} \quad(|I|,|J|=r)
$$

and hence

$$
\sum_{|K|=r} \xi_{K}^{I} S\left(\xi_{J}^{K}\right)=\delta_{I, J} \quad(|I|,|J|=r)
$$

We furthermore recall (cf. [NYM, (3.2)]) that the quantum minors satisfy

$$
\left(\xi_{J}^{I}\right)^{*}=S\left(\xi_{I}^{J}\right)=\frac{\operatorname{sgn}_{q}\left(J ; J^{c}\right)}{\operatorname{sgn}_{q}\left(I ; I^{c}\right)} \xi_{J^{c}}^{I^{c}} \operatorname{det}_{q}^{-1} \quad(|I|=|J|=r),
$$

where $I^{c}:=\{1, \ldots, n\} \backslash I$.

The $A_{q}(U)$-comodule $\Lambda_{q}^{r}(V)$ resp. $\Lambda_{q}^{r}\left(V^{*}\right)$ has highest weight $\Lambda_{r}:=\tilde{\varepsilon}_{1}+\cdots+$ $\tilde{\varepsilon}_{r}$ resp. $\Lambda_{n-r}-\Lambda_{n}=-\tilde{\varepsilon}_{n-r+1}-\cdots-\tilde{\varepsilon}_{n}$ with highest weight vector $v_{1} \wedge \cdots \wedge v_{r}$ resp. $v_{n}^{*} \wedge \cdots \wedge v_{n-r+1}^{*}$. The inner product on $\Lambda_{q}^{r}(V)$ such that $\left\langle v_{I}, v_{J}\right\rangle=\delta_{I, J}$ is $A_{q}(U)$ invariant. On the space $\Lambda_{q}^{r}\left(V^{*}\right)$ we have the invariant inner product $\left\langle v_{I}^{*}, v_{J}^{*}\right\rangle:=$ $\delta_{I, J} q^{-\left\langle 2 \rho, \tilde{\varepsilon}_{I}\right\rangle}$, where $\tilde{\varepsilon}_{I}:=\sum_{i \in I} \tilde{\varepsilon}_{i}$.

Let $U_{q}(\mathfrak{g})=U_{q}(\mathrm{gI}(n, \mathbb{C}))$ denote the quantized universal enveloping algebra (cf. Drinfel'd [Dr], Jimbo $[\mathrm{J}]$ ) associated with the Lie algebra $\mathfrak{g}=\mathrm{gl}(n, \mathbb{C}$ ). In our notation we essentially adhere to Noumi $[\mathrm{N}, \S 1]$. We refer to this last paper for any details not treated here. The algebra $U_{q}(\mathfrak{g})$ is generated by elements $q^{h}$ $(h \in P)$ and $e_{i}, f_{i}(1 \leq i \leq n-1)$ subject to the quantized Weyl-Serre relations.

More useful for the purposes of this paper are the $L$-operators $L_{i j}^{+}, L_{i j}^{-} \in U_{q}(\mathfrak{g})$ $(1 \leq i, j \leq n)$. They generate $U_{q}(\mathfrak{g})$ subject to certain commutation relations that may be conveniently expressed by means of the matrix $R$ defined in (4.1) (cf. $[\mathrm{RTF}])$. The matrices $L^{ \pm}:=\left(L_{i j}^{ \pm}\right)$are upper resp. lower triangular, and $L_{i i}^{ \pm}=q^{ \pm \tilde{\varepsilon}_{i}}$ $(1 \leq i \leq n)$. The Hopf $*$-algebra structure on $U_{q}(\mathfrak{g})$ is uniquely determined by

$$
\Delta\left(L_{i j}^{ \pm}\right)=\sum_{k} L_{i k}^{ \pm} \otimes L_{k j}^{+}, \quad \varepsilon\left(L_{i j}^{ \pm}\right)=\delta_{i j}, \quad\left(L_{i j}^{ \pm}\right)^{*}=S\left(L_{j i}^{\mp}\right) \quad(1 \leq i, j \leq n)
$$

The involution $\tau=* \circ S: U_{q}(\mathfrak{g}) \rightarrow U_{q}(\mathfrak{g})$ acts on the generators as 


$$
\tau\left(L_{i j}^{ \pm}\right)=L_{j i}^{\mp} \quad(1 \leq i, j \leq n) .
$$

There is a natural Hopf $*$-algebra duality $\langle\cdot, \cdot\rangle$ between $U_{q}(\mathrm{~g})$ and $A_{q}(U)$. This means in particular that we have

$$
\left\langle u, \varphi^{*}\right\rangle=\overline{\langle\tau(u), \varphi\rangle}, \quad\left\langle u^{*}, \varphi\right\rangle=\overline{\langle u, \tau(\varphi)\rangle} \quad\left(u \in U_{q}(\mathfrak{g}), \varphi \in A_{q}(U)\right) .
$$

We write $U_{q}(\mathfrak{h})$ for the subalgebra generated by the $q^{h}(h \in P)$. It is Laurent polynomial in the elements $q^{\tilde{\varepsilon}_{i}}(1 \leq i \leq n)$. There is an induced Hopf *-algebra duality between $U_{q}(\mathfrak{h})$ and $A(\mathbb{T})$ such that

$$
\left\langle q^{h}, z^{\lambda}\right\rangle:=q^{\langle h, \lambda\rangle}, \quad z^{\lambda}=z_{1}^{\lambda_{1}} \cdots z_{n}^{\lambda_{n}} \quad(h, \lambda \in P) .
$$

For a right $A_{q}(U)$-comodule $\left(M, \rho_{M}\right)$, the $A_{q}(U)$-coaction $\rho_{M}$ can be "differentiated" using the Hopf algebra pairing $\langle\cdot, \cdot\rangle$. This yields a left $U_{q}(\mathfrak{g})$-module structure on $M$ (cf. $[\mathrm{NYM}]$ ). To be precise, the left $U_{q}(\mathfrak{g})$-action on $M$ is defined by

$$
X \cdot v:=\sum_{(v)}\left\langle X, v_{(2)}\right\rangle_{(1)}, \quad\left(X \in U_{q}(\mathfrak{g}), v \in M\right),
$$

where $\rho_{M}(v)=: \Sigma_{(v)} v_{(1)} \otimes v_{(2)} \in M \otimes A_{q}(U)$ for $v \in M$. For example, differentiating the vector corepresentation (4.4), the corresponding left $U_{q}(\mathfrak{g})$-action gives rise to an algebra homomorphism $\rho_{V}: U_{q}(\mathfrak{g}) \rightarrow \operatorname{End}(V)$, which is uniquely determined by the formulas

$$
R^{ \pm}=\sum_{i j} e_{i j} \otimes \rho_{V}\left(L_{i j}^{ \pm}\right), \quad\left(R^{ \pm}\right)^{-1}=\sum_{i j} e_{i j} \otimes \rho_{V}\left(S\left(L_{i j}^{ \pm}\right)\right) .
$$

Here $\boldsymbol{R}^{-}:=\boldsymbol{R}^{-1}, \boldsymbol{R}^{+}=P R \boldsymbol{P}$, and $\boldsymbol{P}=\sum_{i, j} \boldsymbol{e}_{i j} \otimes \boldsymbol{e}_{j i}$ is the permutation operator. By differentiation of right $A_{q}(U)$-coactions, a 1-1 correspondence is obtained between right $A_{q}(U)$-comodule structures on a finite-dimensional vector space $M$ and $P$ weighted left $U_{q}(\mathrm{~g})$-module structures on $M$. Recall that $M$ is $P$-weighted if it is spanned by vectors that transform under $U_{q}(\mathfrak{h})$ according to $q^{h} \cdot v=q^{\langle h, \lambda\rangle} v(\lambda \in P)$. A highest weight vector $v$ of highest weight $\lambda$ in a left $U_{q}(g)$-module $M$ is then characterized by the conditions $L_{i j}^{-} \cdot v=0(i>j)$ (or, equivalently, $X_{i}^{+} \cdot v=0$ for $i \in[1, n-1])$ and $q^{h} \cdot v=q^{\langle h, \lambda\rangle} v$. There is a similar relationship between left $A_{q}(U)$-comodules and right $U_{q}(\mathfrak{g})$-modules. For a right $U_{q}(\mathfrak{g})$-module $M$, a weight vector $0 \neq v \in M$ is a highest weight vector if $v \cdot L_{i j}^{+}=0(i<j)$ (or, equivalently, $v \cdot X_{i}^{-}=0$ for $i \in[1, n-1]$ ).

Remark 4.2. For a right $A_{q}(U)$-comodule $\left(M, \rho_{M}\right)$ and for an element $v \in M$, we write $v^{\circ}$ when considering $v$ as an element in the left $A_{q}(U)$-comodule $\left(M^{\circ}, \rho_{M}^{\circ}\right)$ (cf. Remark 4.1). Then the differentiated right $U_{q}(\mathrm{~g})$-module structure on $\boldsymbol{M}^{\circ}$ is 
related to the differentiated left $U_{q}(\mathfrak{g})$-module structure on $M$ by $v^{\circ} \cdot X=\left(X^{*} \cdot v\right)^{\circ}$, where $v \in M$ and $X \in U_{q}(\mathrm{~g})$.

The coalgebra structure of $A_{q}(U)$ naturally induces a $A_{q}(U)$-bicomodule structure on $A_{q}(U)$. By differentiating this $A_{q}(U)$-bicomodule structure, $A_{q}(U)$ becomes a $U_{q}(\mathrm{~g})$-bimodule with $U_{q}(\mathrm{~g})$-symmetry. The action of the $L$-operators is then given by

$$
L_{1}^{ \pm} \cdot T_{2}=T_{2} R^{ \pm}, \quad T_{2} \cdot L_{1}^{ \pm}=R^{ \pm} T_{2}
$$

Obviously, the irreducible decomposition of the $U_{q}(\mathfrak{g})$-bimodule $A_{q}(U)$ is given by (4.3). This decomposition may also be characterized as the simultaneous eigenspace decomposition of $A_{q}(U)$ under the action of the center $\mathscr{Z} \subset U_{q}(\mathfrak{g})$.

It can be shown that the pairing $\langle\cdot, \cdot\rangle$ is doubly non-degenerate (cf. [KS, Cor. 23, 54]). In particular, $A_{q}(U)$ can be embedded as Hopf $*$-algebra into the Hopf $*$-algebra dual of $U_{q}(\mathfrak{g})$. The image under this embedding is the Hopf subalgebra spanned by matrix elements of finite-dimensional $P$-weighted $U_{q}(\mathfrak{g})$ modules.

\section{§5. Spherical Corepresentations}

We call $A_{q}(K):=A_{q}(U(n-l)) \otimes A_{q}(U(l))$ the quantized coordinate ring of $K$. Corresponding to the embedding (2.3) there is an obvious surjective Hopf $*$ algebra morphism $\pi_{K}: A_{q}(U(n)) \rightarrow A_{q}(K)$. Write $A_{q}(U / K)$ for the right $A_{q}(K)$ fixed elements in $A_{q}(U)$, i.e.

$$
A_{q}(U / K):=\left\{\varphi \in A_{q}(U) \mid\left(\mathrm{id} \otimes \pi_{K}\right) \circ \Delta(\varphi)=\varphi \otimes 1\right\}
$$

Observe that $A_{q}(U / K)$ is a left $A_{q}(U)$-comodule *-subalgebra of $A_{q}(U)$. The algebra $A_{q}(U / K)$ can be interpreted as a quantized algebra of functions on the complex Grassmannian $U / K$.

For the study of $A_{q}(U / K)$ it is important to obtain explicit information about $A_{q}(K)$-spherical corepresentations of $A_{q}(U)$, i.e. finite-dimensional right $A_{q}(U)$ comodules with non-zero $A_{q}(K)$-fixed vectors. Recall that a vector $v$ in a right $A_{q}(U)$-comodule $M$ with comodule mapping $\rho_{M}: M \rightarrow M \otimes A_{q}(U)$ is $A_{q}(K)$-fixed if

$$
\left(\mathrm{id} \otimes \pi_{K}\right) \circ \rho_{M}(v)=v \otimes 1
$$

One defines $A_{q}(K)$-fixed vectors in left $A_{q}(U)$-comodules in a similar way. In this section we discuss the proof of the following theorem, which states in particular that the pair $\left(A_{q}(U), A_{q}(K)\right)$ is a quantum Gelfand pair : 
Theorem 5.1. Every finite-dimensional irreducible corepresentation of $A_{q}(U)$ has at most one $A_{q}(K)$-fixed vector (up to scalar multiples). The finite-dimensional corepresentations with non-zero $A_{q}(K)$-fixed vectors are parametrized by the classical sublattice $P_{K}^{+}$of spherical dominant weights (cf. §2).

Remark 5.2. Let $M$ be a finite-dimensional right $A_{q}(U)$-comodule. It follows from Remark 4.1 that a vector $v \in M$ is $A_{q}(K)$-fixed if and only if $v \in M^{\circ}$ is $A_{q}(K)$ fixed. Hence, any statement about $A_{q}(K)$-fixed vectors in right $A_{q}(U)$-comodules immediately translates to a corresponding statement for left $A_{q}(U)$-comodules and vice-versa.

For the proof of Theorem 5.1 it suffices to show that the irreducible decomposition of $V_{R}(\lambda)$ as a right $A_{q}(K)$-comodule is the same as the decomposition of the irreducible finite-dimensional representation of $U(n)$ with highest weight $\lambda$ when restricted to the subgroup $K$. One way of establishing this result is by differentiating the coaction of $A_{q}(U)$ on $V_{R}(\lambda)$ using the doubly non-degenerate Hopf algebra pairing between $A_{q}(U)$ and $U_{q}(\mathrm{~g})$. Then the desired result follows from well-known results on the representation theory of quantized universal enveloping algebras. This approach is quite general, and is treated in more detail in [SD].

In this section we discuss another proof of Theorem 5.1 which does not rely on the quantized universal enveloping algebra technique. The strategy will be to relate the decomposition of the restriction to $A_{q}(K)$ of the right $A_{q}(U)$-comodule $V_{R}(\lambda)$ $\left(\lambda \in P^{+}\right)$to characters on the maximal torus $\mathbb{T}$. The following general result about corepresentation theory of semisimple coalgebras is needed (a coalgebra is said to be semisimple if every finite-dimensional $A$-comodule is completely reducible).

Proposition 5.3. Let $A$ and $B$ be semisimple coalgebras. Then every finitedimensional $A \otimes B$-comodule is completely reducible. Write $\left\{V_{\alpha} \mid \alpha \in \hat{A}\right\}$ and $\left\{V_{\beta} \mid \beta \in\right.$ $\hat{B}\}$ for a complete set of mutually inequivalent, irreducible, finite-dimensional right $A$ - and B-comodules, respectively. Then

$$
\left\{V_{\alpha} \otimes V_{\beta} \mid \alpha \in \hat{A}, \beta \in \hat{B}\right\}
$$

is a complete set of mutually inequivalent irreducible finite-dimensional right $A \otimes B$ comodules. Here $V_{\alpha} \otimes V_{\beta}=V_{\alpha} \otimes V_{\beta}$ as a vector space and it has right comodule structure given by $\rho_{\alpha} \otimes \rho_{\beta}:=\sigma_{23} \circ\left(\rho_{\alpha} \otimes \rho_{\beta}\right)$, where $\sigma_{23}$ is the flip of the second and third tensor component and where $\rho_{\alpha}$ and $\rho_{\beta}$ are the right comodule mappings of $V_{\alpha}$ and $V_{\beta}$, respectively.

The proof of the proposition is similar to the analogous, well-known result for tensor products of semisimple algebras and will therefore be omitted here. 
For $\lambda \in P^{+}$with $\lambda_{n} \geq 0$, define the Schur polynomial $s_{\lambda}(z) \in A(\mathbb{T})$ by

$$
s_{\lambda}(z):=\Delta^{-1}(z) \sum_{w \in \subseteq_{n}}(-1)^{l(w)} z^{w(\lambda+\rho)},
$$

with $\Delta(z):=\prod_{i<j}\left(z_{i}-z_{j}\right)$ the Vandermonde determinant. For arbitrary $\lambda \in \mathbb{P}^{+}$with $\lambda_{n} \geq-m(m \in \mathbb{Z})$ define $s_{\lambda}(z):=z^{-m \Lambda_{n}} s_{\lambda+m \Lambda_{n}}(z) \in A(\mathbb{T})$. Then the $s_{\lambda}\left(\lambda \in P^{+}\right)$are well-defined and form a basis of the subalgebra $A(\mathbb{T})^{\mathcal{S}_{n}}$ of symmetric Laurent polynomials. Recall that the character of a finite-dimensional $A_{q}(U)$-comodule $M$ is defined by $\chi_{M}:=\sum_{i} \pi_{i i} \in A_{q}(U)$, where the $\pi_{i j} \in A_{q}(U)$ are the matrix coefficients of $M$ with respect to a basis of $M$. The character $\chi_{M}$ is independent of the particular choice of basis for $M$. As shown in [NYM, (3.22)], the character $\chi_{\lambda} \in A_{q}(U)$ of the irreducible comodule $V_{R}(\lambda)$ satisfies

$$
\left(\chi_{\lambda}\right)_{\mid \mathbb{T}}=s_{\lambda}(z) \quad\left(\lambda \in P^{+}\right),
$$

as in the classical case $(q=1)$.

Proposition 5.4. Let $\lambda \in P^{+}$. The restriction of the $A_{q}(U)$-comodule $V_{R}(\lambda)$ to $A_{q}(K)$ decomposes as

$$
V_{R}(\lambda) \simeq \bigoplus_{\mu, \nu}\left(V_{R}(\mu) \otimes V_{R}(\nu)\right)^{\oplus c_{\mu, \nu}^{\lambda}}
$$

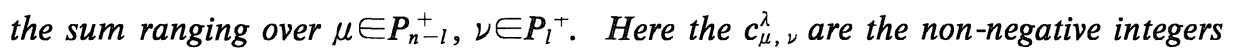
characterized by

$$
s_{\lambda}\left(z_{1}, \ldots, z_{n}\right)=\sum_{\mu, \nu} c_{\mu, \nu}^{\lambda} s_{\mu}\left(z_{1}, \ldots, z_{n-l}\right) s_{\nu}\left(z_{n-l+1}, \ldots, z_{n}\right),
$$

the sum ranging over $\mu \in P_{n-l}^{+}, \nu \in P_{l}^{+}$.

Proof. There exists a decomposition (5.5) for certain uniquely determined non-negative integers $c_{\mu, \nu}^{\lambda}$ by the previous proposition. It follows from (5.4) that the $c_{\mu, \nu}^{\lambda}$ satisfy (5.6), since $\chi_{M \otimes N}=\chi_{M} \otimes \chi_{N} \in A_{q}(K)$ for a finite-dimensional right $A_{q}(U(n-l))$-comodule $M$ and a finite-dimensional right $A_{q}(U(l))$-comodule $N$.

We conclude from Proposition 5.4 that the abstract decomposition of an arbitrary finite-dimensional right $A_{q}(U)$-comodule $M$ into irreducible $A_{q}(K)$-comodules is the same as in the classical $(q=1)$ case. Hence, at this point we see that Theorem 5.1 is a consequence of Theorem 2.1 .

Remark 5.5. The proof of Theorem 5.1 can also be derived from Proposition 5.4 using the Littlewood-Richardson rule (cf. Macdonald [M2]), which is a 
combinatorial rule for computing the coefficients $c_{\mu, \nu}^{\lambda}$ in (5.6).

\section{§6. A One-Parameter Family of Quantum Grassmannians}

In this section we define a family of quantum Grassmannians depending on one real parameter $-\infty<\sigma<\infty$ (cf. [NDS, §2]). The key ingredient in the definition will be the $n \times n$ complex matrix $J^{\sigma}$ defined by

$$
J^{\sigma}:=\sum_{1 \leq k \leq l}\left(1-q^{2 \sigma}\right) e_{k k}+\sum_{l<k<l^{\prime}} e_{k k}-\sum_{1 \leq k \leq l} q^{\sigma} e_{k k^{\prime}}-\sum_{1 \leq k \leq l} q^{\sigma} e_{k^{\prime} k},
$$

where $k^{\prime}:=n-k+1(1 \leq k \leq n)$. Observe that $\lim _{\sigma \rightarrow \infty} J^{\sigma}=J^{\infty}$, where $J^{\infty}$ is defined by

$$
J^{\infty}:=\sum_{k=1}^{n-l} e_{k k}
$$

The subspace $\mathfrak{f}^{\sigma} \subset U_{q}(\mathfrak{g})$ is by definition spanned by the coefficients of the matrix

$$
L^{+} J^{\sigma}-J^{\sigma} L^{-} \in \operatorname{End}(V) \otimes U_{q}(\mathfrak{g}) .
$$

It follows from (4.12) that $\mathfrak{f}^{\sigma}$ is a two-sided coideal in $U_{q}(\mathfrak{g})$, i.e. $\Delta\left(\mathfrak{f}^{\sigma}\right) \subset U_{q}(\mathfrak{g}) \otimes$ $\mathfrak{l}^{\sigma}+\mathfrak{l}^{\sigma} \otimes U_{q}(\mathfrak{g})$ and $\varepsilon\left(\mathfrak{l}^{\sigma}\right)=0$. This remains true when $J^{\sigma}$ is replaced by any $n \times n$ matrix $J$ in the definition of $\mathfrak{l}^{\sigma}$. Moreover, since $J^{\sigma}$ is a symmetric matrix, it follows from (4.13) that $\mathfrak{f}^{\sigma}$ is $\tau$-invariant.

Define the subalgebra $A_{q}\left(\mathfrak{f}^{\sigma} \backslash U\right) \subset A_{q}(U)$ as the subspace of all left $\mathfrak{f}^{\sigma}$-invariant elements in $A_{q}(U)$, i.e. all $a \in A_{q}(U)$ such that $\mathfrak{f}^{\sigma} \cdot a=0$. As is well-known (cf. for instance [DK1, Prop. 1.9]), the fact that $\mathfrak{f}^{\sigma}$ is a $\tau$-invariant two-sided coideal implies the $A_{q}(\mathfrak{f} \backslash \backslash U)$ is a $*$-subalgebra which is invariant under the right $U_{q}(\mathfrak{g})$ action on $A_{q}(U)$ (or, equivalently, the left coaction of $A_{q}(U)$ on itself). Important for the study of $A_{q}\left(\mathfrak{f}^{\sigma} \backslash U\right)$ is the fact that $X=J^{\sigma}$ is a solution of the reflection equation

$$
R_{12} X_{1} R_{12}^{-1} X_{2}=X_{2} R_{21}^{-1} X_{1} R_{21}
$$

where $R_{12}:=R, R_{21}:=P R P\left(=R^{+}\right), X_{1}=X \otimes \mathrm{id}_{V}$ and $X_{2}:=\mathrm{id}_{V} \otimes X$. This fact can be verified by direct computations.

Remark 6.1. The algebra $A_{q}\left(\mathfrak{f}^{\sigma} \backslash U\right)$ can be considered as a quantized coordinate ring on the complex Grassmannian $U(n) /(U(n-l) \times U(l))$ in the following way (see [NDS] for more details). The quantum space of $n \times n$ Hermitean matrices is defined as the algebra generated by $X=\left(x_{i j}\right)_{i j}$ with relations given by the reflection equation (6.4). It can be endowed with a $*$-structure and a left $A_{q}(U)$ - 
coaction (the quantum analogue of the adjoint action). Since $J^{\sigma}$ is a solution of (6.4) it gives rise to a (*-invariant) character of the quantized algebra of functions on the space of Hermitean matrices. In other words, $J^{\sigma}$ corresponds to a classical point in the quantum space of Hermitean matrices. Then $A_{q}(\mathfrak{f} \backslash \backslash U)$ may be considered as the quantized algebra of functions on the adjoint orbit of the classical point corresponding to $J^{\sigma}$ (see [NDS, Prop. 2.4]). Since $J^{\sigma}$ has two different eigenvalues 1 and $-q^{2 \sigma}$ with multiplicity $n-l$ and $l$ respectively, this quantum adjoint orbit is isomorphic with the complex Grassmannian $U(n) /(U(n-l) \times$ $U(l))$.

The quantized function algebra $A_{q}(U / K)$ (5.1) can formally be interpreted as the algebra $A_{q}(\mathfrak{l} ㇒ \backslash U)$ with $\sigma \rightarrow \infty$. To make this a little bit more explicit, we write

$$
L^{+}=\left(\begin{array}{ccc}
{ }^{11} L^{+} & { }^{12} L^{+} & { }^{13} L^{+} \\
0 & { }^{22} L^{+} & { }^{23} L^{+} \\
0 & 0 & { }^{33} L^{+}
\end{array}\right), \quad L^{-}=\left(\begin{array}{cccc}
{ }^{11} L^{-} & 0 & 0 \\
{ }^{21} L^{-} & { }^{22} L^{-} & 0 \\
{ }^{31} L^{-} & { }^{32} L^{-} & { }^{33} L^{-}
\end{array}\right)
$$

where ${ }^{11} L^{+}$is an $l \times l$ matrix, ${ }^{22} L^{+}$an $(n-2 l) \times(n-2 l)$ matrix etc. Let $D$ be the $l \times l$ matrix with 1's on the antidiagonal and 0 's everywhere else. The coefficients of the matrix $L^{+} J^{\sigma}-J^{\sigma} L^{-}$coincide with the coefficients of the following six matrices up to a sign :

$$
\begin{aligned}
& \text { (i ) } q^{\sigma}\left(D \cdot{ }^{31} L^{-}-{ }^{13} L^{+} \cdot D\right)+\left(1-q^{2 \sigma}\right)\left({ }^{11} L^{+}-{ }^{11} L^{-}\right) \\
& \text {(ii) }{ }^{12} L^{+}+q^{\sigma} D \cdot{ }^{32} L^{-} \\
& \text {(iii) }{ }^{23} L^{+} \cdot q^{\sigma} D+{ }^{21} L^{-} \\
& \text {(iv) }{ }^{22} L^{+}-{ }^{22} L^{-} \\
& \text {(v) }{ }^{11} L^{+} \cdot q^{\sigma} D-q^{\sigma} D \cdot{ }^{33} L^{-} \\
& \text {(vi) }{ }^{33} L^{+} \cdot q^{\sigma} D-q^{\sigma} D \cdot{ }^{11} L^{-}
\end{aligned}
$$

Obviously, the coefficients of the following matrix are also contained in $\mathfrak{f}^{\sigma}$ :

$$
\text { (vii) } q^{\sigma}\left(D \cdot{ }^{13} L^{+}-{ }^{31} L^{-} \cdot D\right)+\left(1-q^{2 \sigma}\right)\left({ }^{33} L^{+}-{ }^{33} L^{-}\right) \text {. }
$$

For later use, observe that the following elements of the "Cartan subalgebra" $U_{q}(\mathfrak{h})$ belong to $\mathfrak{l}^{\sigma}$ :

$$
L_{i i}^{+}-L_{i i}^{-} \quad\left(l<i<l^{\prime}\right), \quad L_{i i}^{+}-L_{i^{\prime i^{\prime}}}^{-} \quad(1 \leq i \leq l), \quad L_{i i}^{-}-L_{i i^{\prime}}^{+} \quad(1 \leq i \leq l)
$$

It is clear from (6.5) and (6.6) that, in the limit $\sigma \rightarrow \infty$, the matrices in (i )- 
(vii) tend either to zero or to the following matrices

$$
{ }^{11} L^{+}-{ }^{11} L^{-},{ }^{12} L^{+},{ }^{21} L^{-},{ }^{22} L^{+}-{ }^{22} L^{-},{ }^{33} L^{+}-{ }^{33} L^{-} .
$$

Again, the subspace $\mathfrak{f}^{\infty} \subset U_{q}(\mathfrak{g})$ spanned by the coefficients of the matrices in (6.8) is a $\tau$-invariant two-sided coideal. Now, on the one hand, $\mathfrak{f}^{\infty}$-invariance in a left or right $U_{q}(\mathrm{~g})$-module $M$ is obviously the same as invariance with respect to the Hopf *-subalgebra

$$
U_{q}(\mathfrak{f}):=U_{q}(\mathfrak{g l}(n-l, \mathbb{C})) \otimes U_{q}(\mathfrak{g I}(l, \mathbb{C})) \hookrightarrow U_{q}(\mathfrak{g l}(n, \mathbb{C})),
$$

where invariance of $v \in M$ with respect to $u \in U_{q}(\mathfrak{g})$ should be interpreted as $u \cdot v$ $=\varepsilon(u) \cdot v$ (if $M$ is a left $U_{q}(\mathfrak{g})$-module). Using the Hopf algebra duality between $A_{q}(U)$ and $U_{q}(\mathrm{~g})$ it can be easily shown that invariance of $v \in M$ with respect to $U_{q}(\mathfrak{f})$ is the same as invariance with respect to $A_{q}(K)$ (cf. [DK1, Prop. 1.12]). It follows that $A_{q}(K)$-invariance is equivalent to $\mathfrak{f}^{\infty}$-invariance, hence $A_{q}\left(\mathfrak{f}^{\infty} \backslash U\right)=$ $A_{q}(U / K)$.

Remark 6.2. It should be observed that the matrix $J^{\infty}$ also satisfies the reflection equation (6.4), but the subspace spanned by the coefficients of the matrix $L^{+} J^{\infty}-J^{\infty} L^{-}$is strictly smaller than $\mathfrak{f}^{\infty}$ and of little use for the purposes of this paper.

The following lemma is now a direct consequence of the arguments given above.

Lemma 6.3. Let $M$ be a finite-dimensional right $A_{q}(U)$-comodule with linear basis $\left\{v_{i}\right\}_{i}$. Consider $M$ as left $U_{q}(\mathfrak{g})$-module using the differentiated action (4.15). Suppose that $v_{\sigma}:=\sum_{i} c_{i}(\sigma) v_{i}\left(c_{i}(\sigma) \in \mathbb{C}\right)$ is a $\mathfrak{l}^{\sigma}$-fixed vector for all $\sigma \in \mathbb{R}$ and that $c_{i}:=\lim _{\sigma \rightarrow \infty} c_{i}(\sigma)$ exists for all $i$. Then $\sum_{i} c_{i} v_{i}$ is an $A_{q}(K)$-fixed vector in $M$.

Remark 6.4. In some suitable algebraic sense (cf. [CP, Prop. 9.2.3]) the algebra $U_{q}(\mathrm{~g})$ "tends" to $U_{q}(\mathrm{~g})$ when $q$ tends to 1 . The corresponding limits of the $L$-operators are given by

$$
L_{i j}^{ \pm} /\left(q-q^{-1}\right) \rightarrow \pm e_{j i} \quad(i \lessgtr j), \quad\left(q^{\varepsilon_{i}}-q^{-\varepsilon_{i}}\right) /\left(q-q^{-1}\right) \rightarrow e_{i i}
$$

(cf. $[N,(1.10),(1.11)]$ ). Hence, by (6.8) respectively (6.5), the subspace $\mathfrak{f}^{\sigma} \subset$ $U_{q}(\mathfrak{g})(\sigma=\infty$ respectively $\sigma=0)$ tends to the Lie subalgebra $\mathfrak{f}=\mathfrak{g I}(n-l, \mathbb{C}) \oplus$ $\mathfrak{g l}(l, \mathbb{C}) \subset \mathfrak{g}$ respectively $\mathfrak{f}^{\prime} \subset \mathfrak{g}($ cf. $\S 2)$ in the limit $q \rightarrow 1$.

Reflection equations play an important role in the quantization of symmetric 
spaces (cf. $[\mathrm{N}, \S 2],[\mathrm{NS} 1]$ ). For the purposes of this chapter, the importance of this equation lies in the following fact. Recall that a vector $w$ in a left $U_{q}(\mathrm{~g})$-module $M$ is called $\mathfrak{f}^{\sigma}$-fixed if $\mathfrak{f}^{\sigma} \cdot \mathfrak{w}=0$ (a similar definition can be given for right $U_{q}(\mathfrak{g})$ modules).

Proposition 6.5. ([NS2, Prop. 3.1], [NDS]) Let $J$ be any $n \times n$ complex matrix. Write $\mathfrak{I}^{J} \subset U_{q}(\mathrm{~g})$ for the two-sided coideal spanned by the coefficients of $L^{+} J$ $-J L^{-}$. The element

$$
w^{J}:=\sum_{i, j} J_{i j} v_{i} \otimes v_{j}^{*} \in V \otimes V^{*}
$$

in the left $U_{q}(\mathfrak{g})$-module $V \otimes V^{*}$ is a $\mathfrak{l}^{J}$-fixed vector if and only if $J$ satisfies the reflection equation (6.4).

Proof. In the proof the same notational conventions as in $[\mathrm{N}$, Proof of Proposition 2.3] will be used. Recall that the $U_{q}(\mathrm{~g})$-module structure on $V^{*}$ corresponding to the dual $A_{q}(U)$-comodule $V^{*}$ is given by

$$
u \cdot v^{*}(v):=v^{*}(S(u) \cdot v) \quad\left(u \in U_{q}(\mathfrak{g}), v^{*} \in V^{*}, v \in V\right)
$$

Set $\mathbf{v}:=\left(v_{1}, \ldots, v_{n}\right)$, then it follows from (4.16) that

$$
L_{1}^{ \pm} \cdot \mathbf{v}_{2}=\mathbf{v}_{2} \cdot R_{12}^{ \pm}, L_{1}^{+} \cdot \mathbf{v}_{2}^{*}=\mathbf{v}_{2}^{*} \cdot\left(R_{21}^{-}\right)^{t_{2}}, \quad L_{1}^{-} \cdot \mathbf{v}_{2}^{*}=\mathbf{v}_{2}^{*} \cdot\left(R_{21}^{+}\right)^{t_{2}}
$$

Here $^{t_{2}}$ denotes transposition with respect to the second tensor factor. An equation like $L_{1}^{+} \cdot \mathbf{v}_{2}=\mathbb{v}_{2} \cdot R_{12}^{+}$should be interpreted as $L_{l j}^{+} \cdot v_{k}=\sum_{l=1}^{n}\left(R_{12}^{+}\right)_{j k}^{i l} v_{l}$ for all $1 \leq i, j$, $k \leq n$, where $R_{12}^{+}=\sum_{i, j, k, l}\left(R_{12}^{+}\right)_{j l}^{i k} e_{i j} \otimes e_{k l}$. Using the identities (6.9) one computes in shorthand notation,

$$
\begin{aligned}
L^{+} J \cdot w^{J} & =L_{1}^{+} \cdot\left(\mathbf{v}_{2} J_{2} \otimes\left(\mathbf{v}^{*}\right)_{2}^{t}\right) J_{1}=\left(L_{1}^{+} \cdot \mathbf{v}_{2}\right) J_{2} \otimes L_{1}^{+} \cdot\left(\mathbb{v}^{*}\right)_{2}^{t} J_{1} \\
& =\mathbf{v}_{2} R_{12}^{+} J_{2} R_{21}^{-} J_{1} \otimes\left(\mathbf{v}^{*}\right)_{2}^{t},
\end{aligned}
$$

since by (6.9) one has $L_{1}^{+} \cdot\left(\mathbf{v}^{*}\right)_{2}^{t}=R_{21}^{-} \cdot\left(\mathbf{v}^{*}\right)_{2}^{t}$. On the other hand,

$$
\begin{aligned}
J L^{-} \cdot w^{J} & =J_{1} L_{1}^{-} \cdot\left(\mathbf{v}_{2} J_{2} \otimes\left(\mathbf{v}^{*}\right)_{2}^{t}\right)=J_{1} \mathbf{v}_{2} R_{12}^{-} J_{2} \otimes L_{1}^{-} \cdot\left(\mathbf{v}^{*}\right)_{2}^{t} \\
& =\mathbf{v}_{2} J_{1} R_{12}^{-} J_{2} R_{21}^{+} \otimes\left(\mathbf{v}^{*}\right)_{2}^{t},
\end{aligned}
$$

since by (6.9) one has $L_{1}^{-} \cdot\left(\mathrm{v}^{*}\right)_{2}^{t}=R_{21}^{+}\left(\mathbf{v}^{*}\right)_{2}^{t}$. It follows from the two preceding computations that $w^{J}$ is $\mathfrak{l}^{J}$-fixed if and only if $R_{12}^{+} J_{2} R_{21}^{-} J_{1}=J_{1} R_{12}^{-} J_{2} R_{21}^{+}$. Multiplying this last equation from the left and from the right by the permutation 
operator $P$ gives (6.4), which proves the proposition.

By Proposition 6.5 and the fact that the matrix $J^{\sigma}$ satisfies the reflection equation (6.4), it follows that

$$
w^{\sigma}:=\sum_{i j} J_{i j}^{\sigma} v_{i} \otimes v_{j}^{*} \in V \otimes V^{*}
$$

is a $\mathfrak{f}^{\sigma}$-fixed vector in the left $U_{q}(\mathfrak{g})$-module $V \otimes V^{*}$. Observe that $\lim _{\sigma \rightarrow \infty} w^{\sigma}=w^{\infty}$, with $w^{\infty}$ the right $A_{q}(U / K)$-fixed vector defined by

$$
w^{\infty}:=\sum_{\imath j}^{n} J_{i j}^{\infty} v_{i} \otimes v_{j}^{*}=\sum_{i=1}^{n-l} v_{i} \otimes v_{i}^{*} .
$$

Since $V \otimes V^{*} \simeq V\left(\varpi_{1}\right) \oplus V(0)$ as left $U_{q}(\mathrm{~g})$-modules (where $V(0)$ is the trivial module) and since $w^{\sigma}$ has a non-zero weight component of weight $\varpi_{1}$, it follows that $V\left(\varpi_{1}\right)$ has a non-zero $\mathfrak{f}^{\sigma}$-fixed vector.

Next we construct a right $\mathfrak{f}^{\sigma}$-fixed vector in $V^{\circ} \otimes\left(V^{*}\right)^{\circ}$. Observe that a vector $\tilde{w}=\sum_{i, j} \tilde{J}_{i j} v_{i} \otimes v_{j}^{*} \in V^{\circ} \otimes\left(V^{*}\right)^{\circ}$ for a real matrix $\tilde{J}=\sum_{i j} \tilde{J}_{i j} e_{i j}$ is right $\mathfrak{f}^{\sigma}$-fixed if and only if $\tilde{w}$ is left $S\left(\mathfrak{l}^{\sigma}\right)$-fixed as element in $V \otimes V^{*}$ by the $\tau$-invariance of $\mathfrak{f}^{\sigma}$ and by Remark 4.2. Reasoning as in the proof of Proposition 6.5, it follows that $\tilde{w}$ is left $S\left(\mathfrak{l}^{\sigma}\right)$-fixed if $\widetilde{J}$ is a solution of the linear equation

$$
\boldsymbol{J}_{1}^{\sigma}\left(\boldsymbol{R}_{21}^{-}\right)^{t_{1}} \widetilde{\boldsymbol{J}}_{2}\left(\left(\boldsymbol{R}_{21}^{-}\right)^{t_{1}}\right)^{-1}=\boldsymbol{R}^{t_{1}} \tilde{\boldsymbol{J}}_{2}\left(\boldsymbol{R}^{t_{1}}\right)^{-1} \boldsymbol{J}_{1}^{\sigma}
$$

where $J^{\sigma}$ is given by (6.1). A solution $\tilde{J}=\tilde{J}^{\sigma}$ of (6.12) is given by

$$
\begin{aligned}
\tilde{J}^{\sigma}:=\sum_{1 \leq k \leq l}\left(1-q^{2(n-2 l)} q^{2 \sigma}\right) e_{k k}+\sum_{l<k<l^{\prime}} e_{k k} \\
\quad-q^{\sigma-1} \sum_{1 \leq k \leq l} q^{2(k-l)} e_{k k^{\prime}}-q^{\sigma-1} \sum_{1 \leq k \leq l} q^{2\left(k^{\prime}-l\right)} e_{k^{\prime} k} .
\end{aligned}
$$

We write $\tilde{w}^{\sigma}=\sum_{i j} \tilde{J}_{i j}^{\sigma} v_{i} \otimes v_{j}^{*}$ for the corresponding right $\mathfrak{f}^{\sigma}$-fixed vector in $V^{\circ} \otimes$ $\left(V^{*}\right)^{\circ}$. In the same way as in the case of left $\mathfrak{f}^{\sigma}$-fixed vectors it follows that $V\left(\varpi_{1}\right)^{\circ}$ has a non-zero right $\mathfrak{f}^{\sigma}$-fixed vector. Observe that $\lim _{\sigma \rightarrow \infty} \tilde{\boldsymbol{w}}^{\sigma}=w^{\infty}$, with $w^{\infty}$ the $A_{q}(K)$-fixed vector given by $(6.11)$.

Recall from the previous section that $V(\lambda)$ has at most one $\mathfrak{f}^{\infty}$-fixed vector up to scalar multiples, and that $V(\lambda)$ has non-zero $\mathfrak{f}^{\infty}$-fixed vectors if and only if $\lambda \in$ $P_{K}^{+}$(cf. Theorem 5.1). We have the following analogous statement for $\mathfrak{f}^{\sigma}$-fixed vectors $(-\infty<\sigma<\infty)$.

Theorem 6.6. ([NDS, Thm. 2.6]) Let $\lambda \in \boldsymbol{P}^{+}$and fix $-\infty<\sigma<\infty$. The irreducible left $U_{q}(\mathfrak{g})$-module $V(\lambda)$ with highest weight $\lambda$ has at most one $\mathfrak{l}^{\sigma}$-fixed vector (up to scalar multiples). There exist non-zero $\mathfrak{f}^{\sigma}$-vectors in $V(\lambda)$ if and only if 
$\lambda \in P_{K}^{+}$. The same statement holds for right $\mathfrak{l}^{\sigma}$-fixed vectors in $V(\lambda)^{\circ}$.

In the remainder of this section a proof of Theorem 6.6 will be given. Fix a parameter $-\infty<\sigma<\infty$. First of all, we have the following crucial lemma.

Lemma 6.7. Let $\lambda \in P^{+}$and fix $-\infty<\sigma<\infty$. Then any non-zero $\mathfrak{1}^{\sigma}$-fixed vector in the left $\mathbb{U}_{q}(\mathfrak{g})$-module $V(\lambda)$ has a non-zero weight component of highest weight $\lambda$. The same statement holds for the right $\mathbb{U}_{q}(\mathfrak{g})$-module $V(\lambda){ }^{\circ}$.

The proof of the lemma follows by analyzing the particular form of the twosided coideal $\mathfrak{l}^{\sigma}$. The details are omitted here, since the proof is analogous to the proof of [N, Lemma 3.2] and [DN1, Prop. 3.2].

Since the vector subspace of $V(\lambda)$ (respectively $V(\lambda)^{\circ}$ ) consisting of weight vectors of weight $\lambda$ is one-dimensional, it follows from Lemma 6.7 that every irreducible finite-dimensional $P$-weighted $U_{q}(\mathfrak{g})$-module has at most one $\mathfrak{i}^{\sigma}$-fixed vector up to scalar multiples.

Set $P_{K}=\oplus_{1 \leq r \leq l} \mathbb{Z} \varpi_{r}$, where $\varpi_{r}$ are the fundamental spherical weights (cf. §2). Observe that the assignment $\lambda \longmapsto \lambda^{\natural}$ as defined in $\$ 2$ extends to a bijection from $P_{K}$ onto $P_{\Sigma}$. For $\mu \in P_{\Sigma}$, we write $\mu^{b} \in P_{K}$ for the inverse of $\mu$ under the bijection 手. For later use let us record the following elementary facts. Recall that $\mathscr{W}=\mathscr{W}$ ${ }_{l}$ denotes the Weyl group of the root system $\Sigma$ (cf. §2).

Lemma 6.8. The bijection $\lambda \longmapsto \lambda^{\natural}$ preserves the dominance ordering on $\mathbb{P}_{K}$ and $P_{\Sigma}$. If $\nu \in P_{K}$ then the image under $\lambda \longmapsto \lambda^{\natural}$ of $\left(\Xi_{n} \cdot \nu\right) \cap P_{K}$ is equal to the $\mathscr{W}$ orbit $\mathscr{W} \cdot \nu^{\natural}$ in $P_{\Sigma}$.

The following lemma is immediate from the fact that the Cartan type elements listed in (6.7) belong to $\mathfrak{l}^{\sigma}$.

Lemma 6.9. Let $\lambda \in P_{K}^{+},-\infty<\sigma<\infty$ and assume that $v \in V(\lambda)$ is a non-zero left $\mathfrak{1}^{\sigma}$-fixed vector. Let $v=\sum_{\mu \leq \lambda} v_{\mu}$ be the decomposition of $v$ in weight vectors, where $v_{\mu}$ has weight $\mu \in P$. Then $v_{\mu}=0$ unless $\mu \in P_{K}$. The same statement is valid for the right $U_{q}(\mathfrak{g})$-module $V(\lambda)^{\circ}$.

It follows from Lemma 6.7 and Lemma 6.9 that if $V(\lambda)$ (respectively $V(\lambda)^{\circ}$ ) has a non-zero $\mathfrak{l}^{\sigma}$-fixed vector, then $\lambda \in \mathcal{P}_{K}^{+}$.

To finish the proof of Theorem 6.6 we have to show that all modules $V(\lambda)$ and $V(\lambda)^{\circ}\left(\lambda \in P_{K}^{+}\right)$have non-zero $\mathfrak{l}^{\sigma}$-fixed vectors. The existence of non-trivial $\mathfrak{k}^{\sigma}$ fixed vectors in $V\left(\varpi_{1}\right)$ and in $V\left(\varpi_{1}\right)^{\circ}$ is already proved. Explicit intertwining operators

$$
\hat{\Psi}_{r}:\left(V \otimes V^{*}\right)^{\otimes r} \rightarrow \Lambda_{q}^{r}(V) \otimes \Lambda_{q}^{r}\left(V^{*}\right), \quad(1 \leq r \leq l)
$$


will be constructed to prove the existence of $\mathfrak{l}^{\sigma}$-fixed vectors in higher fundamental spherical representations. The proof of Theorem 6.6 is then completed by computing the so-called principal term of $\hat{\Psi}_{r}\left(\left(w^{\sigma}\right)^{\otimes r}\right)$, with $w^{\sigma} \in V\left(\varpi_{1}\right)$ the $\mathfrak{f}^{\sigma}$-fixed vector given by (6.10).

Before giving the construction of $\hat{\Psi}_{r}$, we first introduce the notion of principal term of a vector $v \in \Lambda_{q}^{r}(V) \otimes \Lambda_{q}^{r}\left(V^{*}\right)$ (cf. [NS2], [Su]). For the present setting it is convenient to use a slightly modified definition of Noumi's and Sugitani's notion of principal term (cf. [NS2], [Su]). The definition is based on certain specific properties of the comodule $\Lambda_{q}^{r}(V) \otimes \Lambda_{q}^{r}\left(V^{*}\right)$. The comodule $\Lambda_{q}^{r}(V) \otimes \Lambda_{q}^{r}\left(V^{*}\right)$ has a multiplicity-free decomposition.

$$
\Lambda_{q}^{r}(V) \otimes \Lambda_{q}^{r}\left(V^{*}\right) \cong \bigoplus_{s=0}^{r} V\left(\varpi_{s}\right) \quad(1 \leq r \leq l)
$$

as right $A_{q}(U)$-comodules, where $\varpi_{0}:=0 \in P_{K}^{+}$. The decomposition (6.14) can be proved by computing the restriction of the character of the module $\Lambda_{q}^{r}(V) \otimes$ $\Lambda_{q}^{r}\left(V^{*}\right)$ to the torus and using the classical Pieri formula for Schur functions [M2, I , (5.17)] (cf. Proposition 5.4). Due to the multiplicity-free decomposition (6.14), the module $\Lambda_{q}^{r}(V) \otimes \Lambda_{q}^{r}\left(V^{*}\right)$ is very useful for the study of $\mathfrak{l}^{\sigma}$-fixed vectors in $V\left(\varpi_{r}\right)$, as will be shown in the remainder of this chapter as well as in the next chapter. It follows from (6.14) that all the weights $\mu \in P$ of the module $\Lambda_{q}^{r}(V) \otimes$ $\Lambda_{q}^{r}\left(V^{*}\right)$ are $\leq \varpi_{r}$, where $\leq$ denotes the dominance order. The vector $v_{[1, r]} \otimes$ $v_{[n-r+1, n]}^{*} \in \Lambda_{q}^{r}(V) \otimes \Lambda_{q}^{r}\left(V^{*}\right)$ is the highest weight vector of the unique copy of $V\left(\varpi_{r}\right)$ within $\Lambda_{q}^{r}(V) \otimes \Lambda_{q}^{r}\left(V^{*}\right)$. Suppose now that $v=\sum_{\mu \leq \sigma_{r}} v_{\mu}$ is the weight space decomposition of a vector $v \in \Lambda_{q}^{r}(V) \otimes \Lambda_{q}^{r}\left(V^{*}\right)$, where $v_{\mu}$ is the weight component of weight $\mu \in P$. Then the principal term of $v$ is defined by

$$
[v]:=\sum_{\nu \in \mathscr{W}\left(1^{r}\right)} v_{\nu^{b}}
$$

(cf. [NS2], $[\mathrm{Su}]$ ), where $\mathscr{W}$ acts on $\left(1^{r}\right) \in P_{\Sigma}^{+} \subset P_{\Sigma}=\mathbb{Z}^{l}$ by permutations and sign changes (cf. §2). It follows from Lemma 6.8 that the principal term of a vector $v$ $\in \Lambda_{q}^{r}(V) \otimes \Lambda_{q}^{r}\left(V^{*}\right)$ lies in the unique copy of $V\left(\varpi_{r}\right)$ within $\Lambda_{q}^{r}(V) \otimes \Lambda_{q}^{r}\left(V^{*}\right)$. By Lemma 6.9 and Lemma 6.8 one has :

Lemma 6.10. Let $v$ be a non-zero $\mathfrak{f}^{\sigma}$-fixed vector in $\Lambda_{q}^{r}(V) \otimes \Lambda_{q}^{r}\left(V^{*}\right)$. If $v$ $[\nu]$ has a non-zero weight component of weight $\nu$ then $\nu \in P_{K}$ and $\nu^{\natural} \in C\left(\varpi_{r}\right)$, where

$$
C(\mu):=\left\{\mu^{\prime} \in P_{\Sigma} \mid w \mu^{\prime}<\mu \forall w \in \mathscr{W}\right\} \quad\left(\mu \in P_{\Sigma}^{+}\right)
$$

is the strict integral convex hull of $\mathscr{W} \mu$.

In the next proposition, the principal term of a $\mathfrak{f}^{\sigma}$-fixed vector in $\Lambda_{q}^{r}(V) \otimes$ $\Lambda_{q}^{r}\left(V^{*}\right)$ (respectively in $\Lambda_{q}^{r}(V)^{\circ} \otimes \Lambda_{q}^{r}\left(V^{*}\right)^{\circ}$ ) is compared with the elements $u_{r}, \tilde{u}_{r}$ 
$(1 \leq r \leq l)$ defined by

$$
u_{r}:=\sum_{\substack{I \subset[1, l] \cup\left[I^{\prime}, n\right] \\|I|=r,, I \cap I^{\prime}=\varnothing}} v_{I} \otimes v_{I^{\prime}}^{*}, \quad \tilde{u}_{r}:=\sum_{\substack{I \subset[1, I] \cup\left[I^{\prime}, n\right] \\|I|=r, I \cap I^{\prime}=\varnothing}} q^{\left\langle 2 \rho, \tilde{E}_{I^{\prime}}\right\rangle} v_{I} \otimes v_{I^{\prime}}^{*}
$$

where $I^{\prime}:=\left\{i^{\prime} \mid i \in I\right\}$. The element $u_{r}$ lies in the unique copy of $V\left(\varpi_{r}\right)$ within $\Lambda_{q}^{r}(V) \otimes \Lambda_{q}^{l}\left(V^{*}\right)$, whereas $\tilde{u}_{r}$ lies in the unique copy of $V\left(\varpi_{r}\right)^{\circ}$ within $\Lambda_{q}^{r}(V)^{\circ} \otimes$ $\Lambda_{q}^{r}\left(V^{*}\right)^{\circ}$. Observe that by the explicit form of the $\mathfrak{f}^{\sigma}$-fixed vectors $w^{\sigma}$ respectively $\tilde{w}^{\sigma}$, we have

$$
\left[w^{\sigma}\right]=-q^{\sigma} u_{1},\left[\tilde{w}^{\sigma}\right]=-q^{\sigma-1} q^{2(1-l)} \tilde{u}_{1}
$$

For the construction of the intertwiner $\hat{\Psi}_{r}$, consider now the linear bijection $\beta: V^{*}$ $\otimes V \rightarrow V \otimes V^{*}$ determined by

$$
\beta\left(v_{i}^{*} \otimes v_{j}\right)=q^{-\delta i j} v_{j} \otimes v_{i}^{*}+\left(q^{-1}-q\right) \delta_{i j} \sum_{k<j} v_{k} \otimes v_{k}^{*} .
$$

Write $V_{i}:=V, V_{i}^{*}:=V^{*}(1 \leq i \leq r)$. Define a linear bijection

$$
\Psi_{r}:\left(V_{1} \otimes V_{1}^{*}\right) \otimes \cdots \otimes\left(V_{r} \otimes V_{r}^{*}\right) \rightarrow\left(V_{1} \otimes \cdots \otimes V_{r}\right) \otimes\left(V_{1}^{*} \otimes \cdots \otimes V_{r}^{*}\right)
$$

by

$$
\Psi_{r}:=\beta_{1, r} \circ \beta_{2, r} \circ \cdots \circ \beta_{r-1, r} \circ \cdots \circ \beta_{13} \circ \beta_{23} \circ \beta_{12},
$$

where $\beta_{i j}$ acts by definition as the identity on all factors of the tensor product except for $V_{i}^{*} \otimes V_{j}$, on which it is equal to $\beta$. Write

$$
\operatorname{pr}_{r}: V^{\otimes r} \rightarrow \Lambda_{q}^{r}(V), \quad \operatorname{pr}_{r}^{*}:\left(V^{*}\right)^{\otimes r} \rightarrow \Lambda_{q}^{r}\left(V^{*}\right)
$$

for the canonical projections. We now have the following generalization of (6.18).

Proposition 6.11. Let $1 \leq r \leq l$. The operator

$$
\hat{\Psi}_{r}:\left(V \otimes V^{*}\right)^{\otimes r} \rightarrow \Lambda_{q}^{r}(V) \otimes \Lambda_{q}^{r}\left(V^{*}\right)
$$

defined by $\hat{\Psi}_{r}:=\left(\mathrm{pr}_{r} \otimes \mathrm{pr}_{r}^{*}\right) \circ \Psi_{r}$ is a surjective intertwiner, and

$$
\begin{aligned}
& {\left[\hat{\Psi}_{r}\left(\left(w^{\sigma}\right)^{\otimes r}\right)\right]=c_{r}(\sigma) u_{r}, \quad c_{r}(\sigma):=\left(\frac{q^{\sigma}}{q^{2}-1}\right)^{r}\left(q^{2} ; q^{2}\right)_{r},} \\
& {\left[\hat{\Psi}_{r}\left(\left(\tilde{w}^{\sigma}\right)^{\otimes r}\right)\right]=\tilde{c}_{r}(\sigma) \tilde{u}_{r}, \quad \tilde{c}_{r}(\sigma):=\left(\frac{q^{\sigma-1} q^{2(1-l)}}{q^{2}-1}\right)^{r}\left(q^{2} ; q^{2}\right)_{r} .}
\end{aligned}
$$


Before giving a proof of Proposition 6.11, we first show it implies Theorem 6.6. Since $\mathfrak{f}^{\sigma}$ is a two-sided coideal and $\Psi_{r}$ an intertwining operator, Proposition 6.11 shows that $\Psi_{r}\left(\left(w^{\sigma}\right)^{\otimes r}\right)$ is a non-zero $\mathfrak{f}^{\sigma}$-fixed vector. Proposition 6.11 implies that the principal term of $\Psi_{r}\left(\left(w^{\sigma}\right)^{\otimes r}\right)$ is non-zero, hence it follows that $V\left(\varpi_{r}\right)(1 \leq r \leq l)$ has a non-zero $\mathfrak{f}^{\sigma}$-fixed vector. Since any $\lambda \in P_{K}^{+}$can be written as a positive integral linear combination of the fundamental spherical weights $\left\{\varpi_{r}\right\}_{1 \leq r \leq l}$, it follows by an easy argument using tensor products and Lemma 6.7 that any $\lambda \in P_{K}^{+}$ is actually spherical. For right $\mathfrak{f}^{\sigma}$-fixed vectors the same argument holds, since $\hat{\Psi}_{r}$ is also an intertwiner as map from the module $\left(V^{\circ} \otimes\left(V^{*}\right)^{\circ}\right)^{\otimes r}$ to $\Lambda_{q}^{r}(V)^{\circ} \otimes$ $\Lambda_{q}^{r}\left(V^{*}\right)^{\circ}$ (cf. Remark 4.1).

So it remains to prove Proposition 6.11. The proof of this proposition, which proceeds by induction on $r$, is broken up into a couple of lemmas.

Lemma 6.12. For $2 \leq r \leq n+1$ the linear mapping

$$
\tilde{\Phi}_{r}: \Lambda_{q}^{r-1}\left(V^{*}\right) \otimes V \rightarrow V \otimes \Lambda_{q}^{r-1}\left(V^{*}\right)
$$

defined on the basis vectors $v_{I}^{*} \otimes v_{j}(|I|=r-1,1 \leq j \leq n)$ by

$$
\tilde{\Phi}_{r}\left(v_{I}^{*} \otimes v_{j}\right)= \begin{cases}v_{j} \otimes v_{I}^{*} & \text { if } j \notin I, \\ q^{-1} v_{j} \otimes v_{I}^{*}-\left(q-q^{-1}\right) \sum_{m<j} \frac{\operatorname{sgn}_{q}(I \backslash j ; m)}{\operatorname{sgn}_{q}(I \backslash j ; j)} v_{m} \otimes v_{(I \backslash j) \cup m}^{*} & \text { if } j \in I\end{cases}
$$

is an intertwining operator of right $A_{q}(U)$-comodules.

Proof. Let $P: V \otimes V \rightarrow V \otimes V$ denote the flip. Define a linear bijection $\gamma: V$ $\otimes V \rightarrow V \otimes V$ by $\gamma:=P R$, with $R$ as in (4.1). The action of $\gamma$ on the basis vectors $v_{i} \otimes v_{j}(1 \leq i, j \leq n)$ is given by

$$
\gamma\left(v_{i} \otimes v_{j}\right)=q^{\delta_{i j}} v_{j} \otimes v_{i}+\left(q-q^{-1}\right) \theta_{i, j} v_{i} \otimes v_{j}
$$

with $\theta_{i, j}:=1$ if $i<j$ and $\theta_{i, j}:=0$ otherwise. The fact that the commutation relations between the $t_{i j} \in A_{q}(U)$ can be written as $R T_{1} T_{2}=T_{2} T_{1} R$ (cf. §3) implies that $\gamma$ is an intertwining operator. Since $R$ is a solution of the Quantum Yang-Baxter Equation, $\gamma$ satisfies

$$
\gamma_{1} \circ \gamma_{2} \circ \gamma_{1}=\gamma_{2} \circ \gamma_{1} \circ \gamma_{2}
$$

with $\gamma_{i} \in \operatorname{End}\left(V^{\otimes 3}\right)$ acting as $\gamma$ on the $i$ th and $(i+1)$ th tensor factors and as the identity on the remaining factor. Note furthermore that the exterior algebra $\Lambda_{q}(V)$ 
is isomorphic as a right $A_{q}(U)$-comodule algebra with $T(V) / I$, where $T(V)$ is the tensor algebra of $V$ and $I \subset T(V)$ the two-sided ideal generated by $\operatorname{ker}\left(\right.$ id $\left.-q^{-1} \gamma\right) \subset$ $V^{\otimes 2} \subset T(V)$. Consider now the intertwiner $\Gamma_{k}: V^{\otimes(k-1)} \otimes V \rightarrow V \otimes \Lambda_{q}^{k-1}(V)(2 \leq k$ $\leq n+1)$ defined by

$$
\Gamma_{k}=\left(\mathrm{id} \otimes \mathrm{pr}_{k-1}\right) \circ \gamma_{1} \circ \gamma_{2} \circ \cdots \circ \gamma_{k-1}
$$

Application of $[\mathrm{HH}$, Lemma $4.9(1)]$ to the Yang-Baxter operator $q^{-1} \gamma$ shows that there exists a unique bijective intertwiner

$$
\hat{\Gamma}_{k}: \Lambda_{q}^{k-1}(V) \otimes V \rightarrow V \otimes \Lambda_{q}^{k-1}(V)
$$

such that $\Gamma_{k}=\hat{\Gamma}_{k} \circ\left(\mathrm{pr}_{k-1} \otimes \mathrm{id}\right)$. By a straightforward computation one verifies that

$$
\begin{aligned}
\hat{\Gamma}_{k}\left(v_{I} \otimes v_{j}\right) & =q^{|I \cap j|} v_{j} \otimes v_{I}+ \\
& +\left(1-q^{2}\right)(-q)^{-k+1} \operatorname{sgn}_{q}(I ; j) \sum_{\substack{i \in I \\
i<j}} \operatorname{sgn}_{q}(i ; I \backslash i) v_{i} \otimes v(I \backslash i) \cup j
\end{aligned}
$$

for $I \subset[1, n]$ with $|I|=k-1$ and $1 \leq j \leq n$.

Next, the linear mapping $\delta_{k}: \Lambda_{q}^{k}\left(V^{*}\right) \rightarrow \Lambda_{q}^{n-k}(V) \otimes \mathbb{C} \operatorname{det}_{q}^{-1}(1 \leq k \leq n)$ defined on the basis elements $v_{I}^{*}(|I|=k)$ by $\delta_{k}\left(v_{I}^{*}\right):=\operatorname{sgn}_{q}\left(I ; I^{c}\right) v_{I^{c}} \otimes \operatorname{det}_{q}^{-1}$ is a bijective intertwiner by (4.11). With the canonical identification $V \otimes \mathbb{C} \operatorname{det}_{q}^{-1} \cong$ $\mathbb{C} \operatorname{det}_{q}^{-1} \otimes V$ we have an intertwining operator $\hat{\Phi}_{r}: \Lambda_{q}^{r-1}\left(V^{*}\right) \otimes V \rightarrow V \otimes \Lambda_{q}^{r-1}\left(V^{*}\right)$ defined by

$$
\tilde{\Phi}_{r}:=q^{-1}\left(\mathrm{id} \otimes \delta_{r-1}^{-1}\right) \circ\left(\hat{\Gamma}_{n-r+2} \otimes \mathrm{id}\right) \circ\left(\delta_{r-1} \otimes \mathrm{id}\right)
$$

Starting from the explicit expressions for $\hat{\Gamma}_{n-r+2}$ and $\delta_{r-1}$, a straightforward calculation shows that $\tilde{\Phi}_{r}$ acts on the basis vectors $v_{I}^{*} \otimes v_{j}$ as required.

Corollary 6.13. The linear mappings $\beta, \Psi_{r}$, and $\hat{\Psi}_{r}$ are right $A_{q}(U)$-comodule homomorphisms.

Proof. The assertion follows from the previous lemma, since $\beta=\hat{\Phi}_{2}$ and the natural projections $\mathrm{pr}_{r}$ and $\mathrm{pr}_{r}^{*}$ intertwine the right $A_{q}(U)$-comodule actions.

Lemma 6.14. Let $1 \leq r \leq l$. The bijective intertwining operator

$$
\Phi_{r}:\left(V_{1}^{*} \otimes \cdots \otimes V_{r-1}^{*}\right) \otimes V_{1} \rightarrow V_{1} \otimes\left(V_{1}^{*} \otimes \cdots \otimes V_{r-1}^{*}\right)
$$

defined by $\Phi_{r}:=\beta_{12} \circ \beta_{23} \circ \cdots \circ \beta_{r-2, r-1} \circ \beta_{r-1, r}$ satisfies 


$$
\left(\mathrm{id} \otimes \mathrm{pr}_{r-1}^{*}\right) \circ \Phi_{r}=\hat{\Phi}_{r} \circ\left(\mathrm{pr}_{r-1}^{*} \otimes \mathrm{id}\right) .
$$

Proof. For $I=\left\{i_{1}<\cdots<i_{r}\right\} \subset[1, n]$, set $\tilde{v}_{I}^{*}:=v_{i_{r}}^{*} \otimes \cdots \otimes v_{i_{2}}^{*} \otimes v_{i_{1}}^{*}$. It is clear from the definitions that

$$
\left(\mathrm{id} \otimes \operatorname{pr}_{r-1}^{*}\right) \circ \Phi_{r}\left(\tilde{v}_{I}^{*} \otimes v_{j}\right)=v_{j} \otimes v_{I}^{*} \quad \text { if } j \not \subset I .
$$

If $j \in I$, then

$$
\left(\mathrm{id} \otimes \mathrm{pr}_{r-1}^{*}\right) \circ \Phi_{r}\left(\tilde{v}_{I}^{*} \otimes v_{j}\right)=q^{-1} v_{j} \otimes v_{I}^{*}-\left(q-q^{-1}\right) \sum_{m<j} c(m, j) v_{m} \otimes v_{(I \backslash j) \cup m}^{*},
$$

where $c(m, j):=(-q)^{|\{i \in I \mid m<i<j\}|}$ if $m \notin \subset I$, and $c(m, j):=0$ otherwise. Using the definition of the $q$-signum $\operatorname{sgn}_{q}$, it follows that $c(m, j)=\operatorname{sgn}_{q}(I \backslash j ; m) \operatorname{sgn}_{q}(I \backslash j$; $j)^{-1}$ if $m<j$, which concludes the proof of the lemma.

Observe that the multiplication maps

$$
\mu: \Lambda_{q}(V) \otimes \Lambda_{q}(V) \rightarrow \Lambda_{q}(V), \quad \mu^{*}: \Lambda_{q}\left(V^{*}\right) \otimes \Lambda_{q}\left(V^{*}\right) \rightarrow \Lambda_{q}\left(V^{*}\right)
$$

are intertwiners of the $A_{q}(U)$-coactions, since $\Lambda_{q}(V)$ and $\Lambda_{q}\left(V^{*}\right)$ are $A_{q}(U)$ comodule algebras.

\section{Lemma 6.15. The intertwining operator}

$$
\hat{\Theta}_{r}: \Lambda_{q}^{r-1}(V) \otimes \Lambda_{q}^{r-1}\left(V^{*}\right) \otimes V \otimes V^{*} \rightarrow \Lambda_{q}^{r}(V) \otimes \Lambda_{q}^{r}\left(V^{*}\right)
$$

defined by $\hat{\Theta}_{r}:=\left(\mu \otimes \mu^{*}\right) \circ\left(\operatorname{id}_{\Lambda_{q}^{r-1}(V)} \otimes \hat{\Phi}_{r} \otimes \mathrm{id}_{V^{*}}\right)$ satisfies

$$
\begin{aligned}
& {\left[\hat{\Theta}_{r}\left(u_{r-1} \otimes w^{\sigma}\right)\right]=-q^{\sigma} \frac{1-q^{2 r}}{1-q^{2}} u_{r}} \\
& {\left[\hat{\Theta}_{r}\left(\tilde{u}_{r-1} \otimes \tilde{w}^{\sigma}\right)\right]=-q^{\sigma-1} q^{2(1-l)} \frac{1-q^{2 r}}{1-q^{2}} \tilde{u}_{r}}
\end{aligned}
$$

for $2 \leq r \leq l$.

Proof. If $v$ is a vector of weight $\mu$ in the domain of $\hat{\Theta}_{r}$, then $\hat{\Theta}_{r}(v)$ is again a weight vector of weight $\mu$, since $\hat{\Theta}_{r}$ intertwines the right $A_{q}(U)$-coaction. Hence, for a fixed $I \subset[1, l] \cup\left[l^{\prime}, n\right]$ with $I \cap I^{\prime}=\varnothing$ and $|I|=r-1$, we have that $\left[\hat{\Theta}_{r}\left(v_{I} \otimes\right.\right.$ $\left.\left.v_{I^{\prime}}^{*} \otimes v_{s} \otimes v_{t}^{*}\right)\right]=0$ unless $s, t \not \subset I \cup I^{\prime}$ and $s \neq t$. By the explicit formulas for the action of $\hat{\Phi}_{r}$ (cf. Lemma 6.12), it follows that 


$$
\left[\hat{\Theta}_{r}\left(u_{r-1} \otimes w^{\sigma}\right)\right]=-q^{\sigma} \sum_{I, k} v_{I} \wedge v_{k} \otimes v_{I^{\prime}}^{*} \wedge v_{k^{\prime}}^{*}=-q^{\sigma} \sum_{J} c_{J} v_{J} \otimes v_{J^{\prime}}^{*}
$$

where the first sum is taken over pairs $(I, k)$ with $I \subset[1, l] \cup\left[l^{\prime}, n\right], k \in[1, l] \cup$ $\left[l^{\prime}, n\right],|I|=r-1, I \cap I^{\prime}=\varnothing$ and $k \not \subset I \cup I^{\prime}$, and the second sum is taken over subsets $J \subset[1, l] \cup\left[l^{\prime}, n\right]$ with $J \cap J^{\prime}=\varnothing$ and $|J|=r$. The corresponding constant $c_{J}$ is given by

$$
c_{J}=\sum_{k \in J} \operatorname{sgn}_{q}(J \backslash k ; k) \operatorname{sgn}_{q}\left(k^{\prime} ; J^{\prime} \backslash k^{\prime}\right)=\sum_{k \in J}\left(\operatorname{sgn}_{q}(J \backslash k ; k)\right)^{2}=\sum_{s=0}^{r-1} q^{2 s}=\frac{1-q^{2 r}}{1-q^{2}} .
$$

The proof for the leading term of $\hat{\Theta}_{r}\left(\tilde{u}_{r-1} \otimes \tilde{w}^{\sigma}\right)$ is similar.

Proposition 6.11 can now be proved by induction to $r$, using the previous lemma for the induction step.

Proof of Proposition 6.11. Define an intertwiner

$$
\Theta_{r}: V^{\otimes(r-1)} \otimes\left(V^{*}\right)^{\otimes(r-1)} \otimes V \otimes V^{*} \rightarrow V^{\otimes r} \otimes\left(V^{*}\right)^{\otimes r}
$$

by

$$
\Theta_{r}:=\mathrm{id}_{V^{\otimes(r-1)}} \otimes \Phi_{r} \otimes \mathrm{id}_{V^{*}}
$$

It follows from Lemma 6.14 that

$$
\left(\mathrm{pr}_{r} \otimes \mathrm{pr}_{r}^{*}\right) \circ \Theta_{r}=\hat{\Theta}_{r} \circ\left(\mathrm{pr}_{r-1} \otimes \mathrm{pr}_{r-1}^{*} \otimes \mathrm{id}_{V} \otimes \mathrm{id}_{V^{*}}\right)
$$

From the definitions of $\Psi_{r}$ and $\Phi_{r}$ it follows that

$$
\Psi_{r}=\Theta_{r} \circ\left(\Psi_{r-1} \otimes \mathrm{id}\right)
$$

and hence by (6.23)

$$
\hat{\Psi}_{r}=\hat{\Theta}_{r} \circ\left(\hat{\Psi}_{r-1} \otimes \mathrm{id}\right)
$$

This allows us to prove the proposition by induction to $r$. The proposition is trivial for $r=1$. Suppose that $r \geq 2$. By the induction hypotheses and Lemma 6.10 we have

$$
\hat{\Psi}_{r-1}\left(\left(w^{\sigma}\right)^{\otimes r-1}\right)=c_{r-1}(\sigma) u_{r-1}+\sum_{\nu \in C\left(\left(1^{r-1}\right)\right)} v_{\nu^{b}}
$$

where $\nu_{\nu^{b}}$ is some weight vector of weight $\nu^{b}$ and $C(\mu)$ is defined by (6.16). For 
$\nu \in C\left(\left(1^{r-1}\right)\right)$ we have $\left[\hat{\Theta}_{r}\left(\nu_{\nu} b \otimes w^{\sigma}\right)\right]=0$, hence the induction step for computation of $\left[\hat{\Psi}_{r}\left(\left(w^{\sigma}\right)^{\otimes r}\right)\right]$ follows by combining Lemma 6.15 with (6.24). The leading term $\left[\hat{\Psi}_{r}\left(\left(\tilde{\boldsymbol{w}}^{\sigma}\right)^{\otimes r}\right)\right]$ can be computed in a similar way.

Remark 6.16. It should be observed that the proof of Theorem 6.6 differs in important details from the proof of Theorem 5.1. Observe for instance that Lemma 6.7 does not hold with $\mathfrak{f}^{\sigma}$-fixed replaced by $\mathfrak{f}^{\infty}$-fixed, since any $\mathfrak{f}^{\infty}$-fixed vector lies automatically in the zero weight space of the module.

\section{§7. Zonal $(\sigma, \tau)$-Spherical Functions}

In this section the $\mathfrak{f}^{\tau}$-invariant $(-\infty<\tau<\infty)$ functions are studied in the quantized coordinate ring $A_{g}\left(\mathfrak{l}^{\sigma} \backslash U\right)(-\infty<\sigma<\infty)$. The results of this section were announced in [NDS, §3]. The rank 1 case of these results were earlier derived by Koornwinder $[\mathrm{K} 1]$ for $n=2$ and for arbitrary complex projective space by Noumi and Dijkhuizen [DN1].

Let $-\infty<\sigma, \tau \leq \infty$ and denote $\mathscr{H}^{\sigma, \tau}$ for the $*$-subalgebra of left $\mathfrak{f}^{\sigma}$-invariant and right $\mathfrak{f}^{\tau}$-invariant function in $A_{q}(U)$. From Theorem 5.1, Theorem 6.6 and (4.3) we obtain the decomposition

$$
\mathscr{H}^{\sigma, \tau}=\underset{\lambda \in P_{x}^{+}}{\bigoplus} \mathscr{H}^{\sigma, \tau}(\lambda), \quad \mathscr{H}^{\sigma, \tau}(\lambda):=W(\lambda) \cap \mathscr{H}^{\sigma, \tau},
$$

the subspaces $\mathscr{H}^{\sigma, \tau}(\lambda)\left(\lambda \in P_{K}^{+}\right)$being one-dimensional. A non-zero element $\varphi^{\sigma, \tau}(\lambda) \in \mathscr{H}^{\sigma, \tau}(\lambda)$ is called a zonal $(\sigma, \tau)$-spherical function. Since the decomposition (4.3) is orthogonal with respect to the inner product $\langle\varphi, \phi\rangle=$ $h\left(\phi^{*} \varphi\right)$, the zonal spherical functions $\varphi^{\sigma, \tau}(\lambda)\left(\lambda \in P_{K}^{+}\right)$are mutually orthogonal with respect to $\langle\cdot, \cdot\rangle$.

Let $M$ denote a right $A_{q}(U)$-comodule with comodule mapping $\rho_{M}$ and an invariant inner product $\langle\cdot, \cdot\rangle$. With any two elements $v, w \in M$ we associate the matrix coefficient

$$
\theta_{M}(v, w):=\sum_{(w)}\left\langle w_{(1)}, v\right\rangle_{w_{(2)}} \in A_{q}(U), \quad \rho_{M}(w):=\sum_{(w)} w_{(1)} \otimes w_{(2)}
$$

The map $\theta_{M}$ induces a linear map (denoted by the same symbol)

$$
\theta_{M}: M^{\circ} \otimes M \rightarrow A_{q}(U)
$$

which is surjective onto the subspace spanned by the matrix coefficients of $M$. If no confusion can arise we sometimes write $\theta:=\theta_{M}$. The following lemma is a direct consequence of these definitions (cf. [N, Lemma 4.8]). 
Lemma 7.1. Let $M$ be a unitary right $A_{q}(U)$-comodule. The map $\theta_{M}: M^{\circ} \otimes$ $M \rightarrow A_{q}(U)$ satisfies the following properties :

(i ) $\theta_{M}$ is a $A_{q}(U)$-bicomodule homomorphism, i.e.

$$
\Delta \circ \theta_{M}=\left(\theta_{M} \otimes \mathrm{id}\right) \circ\left(\mathrm{id} \otimes \rho_{M}\right), \quad \Delta \circ \theta_{M}=\left(\mathrm{id} \otimes \theta_{M}\right) \circ\left(\rho_{M}^{\circ} \otimes \mathrm{id}\right),
$$

where $\rho_{M}^{\circ}$ is defined as in Remark 4.1.

(ii) $\theta_{M}(v, w)=\tau\left(\theta_{M}(w, v)\right)(v, w \in M)$.

(iii) If $M$ is irreducible of highest weight $\lambda \in P^{+}$, then $\theta_{M}: M^{\circ} \otimes M \rightarrow W(\lambda)$ is an isomorphism of $A_{q}(U)$-bicomodules.

Lemma 7.1 can be used to construct zonal $(\sigma, \tau)$-spherical functions as follows. Let $v_{\sigma}(\lambda) \in V(\lambda)$ respectively $\tilde{v}_{\tau}(\lambda) \in V(\lambda)^{\circ}$ be a non-zero $\mathfrak{f}^{\sigma}$-fixed respectively $\mathfrak{l}^{\tau}$-fixed vector $\left(\lambda \in \mathbb{P}_{K}^{+}\right)$. Let $\langle\cdot, \cdot\rangle$ be a positive definite inner product on $V(\lambda)$, and write $\theta_{\lambda}$ for the map $\theta$ in Lemma 7.1 with respect to the unitary comodule $(V(\lambda),\langle\cdot, \cdot\rangle)$. Then

$$
\varphi^{\sigma, \tau}(\lambda):=\theta_{\lambda}\left(\tilde{v}_{\tau}(\lambda), v_{\sigma}(\lambda)\right) \in \mathscr{H}^{\sigma, \tau}(\lambda)
$$

is a zonal $(\sigma, \tau)$-spherical function by Lemma 7.1. This leads to the following lemma.

Lemma 7.2. Let $-\infty<\sigma, \tau<\infty$ and $\lambda \in P_{K}^{+}$. The image of $\varphi^{\sigma, \tau}(\lambda)$ under the restriction map ${ }_{\mid \mathrm{T}}: A_{q}(U) \rightarrow A(\mathbb{T})$ is of the form

$$
\varphi^{\sigma, \tau}(\lambda)_{\mid \mathbb{T}}=c_{\lambda^{\natural}} m_{\lambda^{\natural}}(x)+\sum_{\nu \in C\left(\lambda^{\natural}\right)} c_{\nu} x^{\nu}, \quad c_{\nu} \in \mathbb{C},
$$

with $c_{\lambda} \neq 0$ and $C(\nu)$ given by (6.16). Here the notation $x^{\nu}:=x_{1}^{\nu_{1}} x_{2}^{\nu_{2}} \cdots x_{l}^{\nu}$ for $\nu=$ $\left(\nu_{1}, \ldots, \nu_{l}\right) \in P_{\Sigma}$ is used, with the $x_{i}(1 \leq i \leq l)$ being defined by (2.10).

Proof. Since any $\lambda \in{P_{K}^{+}}^{+}$can be written as a positive integral linear combination of the fundamental spherical weights $\left\{\varpi_{r}\right\}_{1 \leq r \leq l}$, it follows by an easy argument using tensor products and Lemma 6.7 that (7.5) for arbitrary $\lambda \in P_{K}^{+}$follows from (7.5) for the fundamental spherical weights $\left\{\varpi_{r}\right\}_{r=1}^{l}$.

So fix a fundamental weight $\varpi_{r}(1 \leq r \leq l)$. Consider the $A_{q}(U)$-invariant inner product

$$
\left\langle v_{I} \otimes v_{J}^{*}, v_{K} \otimes v_{L}^{*}\right\rangle=q^{-\left\langle 2 \rho, \tilde{\varepsilon}_{J}\right\rangle} \delta_{I, K} \delta_{J, L}
$$

on $\Lambda_{q}^{r}(V) \otimes \Lambda_{q}^{r}\left(V^{*}\right)$ (cf. $\S 3$ ) and write $\theta$ for the map (7.2) associated with the unitary comodule $\left(\Lambda_{q}^{r}(V) \otimes \Lambda_{q}^{r}\left(V^{*}\right),\langle\cdot, \cdot\rangle\right)$. By (6.14), the comodule $V\left(\varpi_{r}\right)$ may be considered as an irreducible component of $\Lambda_{q}^{r}(V) \otimes \Lambda_{q}^{r}\left(V^{*}\right)$ with invariant 
inner product given by the restriction of $\langle\cdot, \cdot\rangle$ to $V\left(\varpi_{r}\right)$. Then, by Proposition 6.11 and the fact that $u_{r} \in \Lambda_{q}^{r}(V) \otimes \Lambda_{q}^{r}\left(V^{*}\right)\left(\right.$ respectively $\left.\tilde{u}_{r} \in \Lambda_{q}^{r}(V)^{\circ} \otimes \Lambda_{q}^{r}\left(V^{*}\right)^{\circ}\right)$ lies in the unique irreducible component $V\left(\varpi_{r}\right)$ (respectively $V\left(\varpi_{r}\right)^{\circ}$ ), the principal terms of the $\mathfrak{f}^{\sigma}$-fixed vector $v_{o}\left(\varpi_{r}\right)$ and the $\mathfrak{l}^{\tau}$-fixed vector $\tilde{v}_{r}\left(\varpi_{r}\right)$ are given by

$$
\left[v_{\sigma}\left(\varpi_{r}\right)\right]=c_{r} u_{r}, \quad\left[\tilde{v}_{\tau}\left(\varpi_{r}\right)\right]=\tilde{c}_{r} \tilde{u}_{r}
$$

for non-zero constants $c_{r}, \tilde{c}_{r} \in \mathbb{C}$. For $u_{\mu} \in \Lambda_{q}^{r}(V) \otimes \Lambda_{q}^{r}\left(V^{*}\right)$ of weight $\mu$ and $\tilde{v}_{\nu} \in$ $\Lambda_{q}^{r}(V)^{\circ} \otimes \Lambda_{q}^{r}\left(V^{*}\right)^{\circ}$ of weight $\nu$ we have $\theta\left(\tilde{v}_{\nu}, v_{\mu}\right)_{\mid \mathbb{T}}=0$ if $\mu \neq \nu$, and $\theta\left(\tilde{v}_{\mu}, v_{\mu}\right)_{\mid \mathrm{T}}$ is a multiple of $z^{\mu}$. Using Lemma 6.9 and the fact that $\mathbb{C}\left[x^{ \pm 1}\right]$ is the subalgebra of $A$ (T) spanned by the monomials $z^{\mu}\left(=x^{\mu^{\natural}}\right)\left(\mu \in P_{K}\right)$, we obtain from (7.7) that

$$
\begin{aligned}
\varphi^{\sigma, \tau}(\lambda)_{\mid \mathrm{T}} & =\theta\left(\tilde{v}_{\tau}\left(\varpi_{r}\right), v_{\sigma}\left(\varpi_{r}\right)\right)_{\mid \mathrm{T}} \\
& =\theta\left(\left[\tilde{v}_{\tau}\left(\varpi_{r}\right)\right],\left[v_{\sigma}\left(\varpi_{r}\right)\right]\right)_{\mid \mathrm{T}}+\sum_{\nu \in\left(\left(1^{r}\right)\right)} d_{\nu} x^{\nu} \\
& =d_{\left(1^{r}\right)} m_{\left(1^{r}\right)}(x)+\sum_{\nu \in C\left(\left(1^{r}\right)\right)} d_{\nu} x^{\nu}
\end{aligned}
$$

with $d_{\left(1^{r}\right)}=c_{r} \tilde{c}_{r} \neq 0$, since $\theta\left(\tilde{u}_{r}, u_{r}\right)_{\mid \mathrm{T}}=m_{\left(1^{r}\right)}(x)$. This completes the proof of (7.5) for the fundamental spherical weights.

Lemma 7.2 has the following important consequence.

Corollary 7.3. The restriction of the map $\left.\right|_{\mathbb{T}}: A_{q}(U) \rightarrow A(\mathbb{T})$ to $\mathscr{H}^{\sigma, \tau}$ defines an injection from $\mathscr{H}^{\sigma, \tau}$ into $\mathbb{C}\left[x^{ \pm 1}\right]$ for $-\infty<\sigma, \tau<\infty$. In particular, $\mathscr{H}^{\sigma, \tau}$ is a commutative algebra for $-\infty<\sigma, \tau<\infty$.

Recall from $[\mathrm{RTF}]$ and $[\mathrm{NDS}, \S 3]$ the Casimir operator

$$
C:=\sum_{\imath j} q^{2(n-l)} L_{i j}^{+} S\left(L_{j i}^{-}\right) \in U_{q}(\mathfrak{g}) .
$$

Since $C$ is central, it acts on $W(\lambda)\left(\lambda \in P^{+}\right)$as a scalar $\chi_{\lambda}(C)$, which is given by

$$
\chi_{\lambda}=\sum_{k=1}^{n} q^{2\left(\lambda_{k}+n-k\right)} .
$$

Also, $C$ maps $\mathscr{H}^{\sigma, \tau}$ into itself. Therefore, if $-\infty<\sigma, \tau<\infty$, the restricted Casimir operator $C: \mathscr{H}^{\sigma, \tau} \rightarrow \mathscr{H}^{\sigma, \tau}$ induces an operator

$$
L: \mathscr{H}^{\sigma, \tau}{ }_{\mid \mathbb{T}} \rightarrow \mathscr{H}^{\sigma, \tau}{ }_{\mid \mathbb{T}} \subset \mathbb{C}\left[x^{ \pm 1}\right]
$$


which is called the radial part of $C$. Explicitly, $L$ is the map satisfying

$$
L\left(\varphi_{\mid \mathrm{T}}\right)=(C \varphi)_{\mid \mathrm{T}}, \quad \forall \varphi \in \mathscr{H}^{\sigma, \tau}
$$

Crucial for the identification of the zonal $(\sigma, \tau)$-spherical functions is the identification of the radial part $L$ of the Casimir element $C$ with the restriction to $\mathscr{H}^{\sigma, \tau}{ }_{\mid \mathrm{T}}$ of an explicit second-order $q^{2}$-difference operator on $\mathbb{C}\left[x^{ \pm 1}\right]$. Without proof we will state here the result (see [NDS, §3]).

Theorem 7.4. ( $[\mathrm{NDS}]$ ) Let $-\infty<\sigma, \tau<\infty$ and $\lambda \in \boldsymbol{P}_{K}^{+}$. The operator $L-$ $\chi_{\lambda}(C)$ id coincides on $\mathscr{H}^{\sigma, \tau}{ }_{\mid \mathbb{T}} \subset \mathbb{C}\left[x^{ \pm 1}\right]$ with a constant multiple of Koornwinder's second-order $q^{2}$-difference operator $D-E_{\lambda}$ id in the variables $x=\left(x_{1}, \ldots, x_{l}\right)$ with base $q^{2}$ and parameters $(\underline{t}, t)=\left(\underline{t}^{\sigma, \tau}, q^{2}\right)$, given by

$$
\begin{aligned}
t_{0}^{\sigma, \tau}=-q^{\sigma+\tau+1}, & t_{1}^{\sigma, \tau}=-q^{-\sigma-\tau+1}, \\
t_{2}^{\sigma, \tau}=q^{\sigma-\tau+1}, & t_{3}^{\sigma, \tau}=q^{-\sigma+\tau+2(n-2 l)+1} .
\end{aligned}
$$

For a proof of the theorem for rank 1, see [DN1]. In [NS1] a proof can be found for the special case $n=2 l$ and $\sigma=\tau=0$.

Observe that for $-\infty<\sigma, \tau<\infty$ we have $\underline{t}^{\sigma, \tau} \in V_{K}$ (cf. §3) and $t_{0}^{\sigma, \tau} t_{1}^{\sigma, \tau} t_{2}^{\sigma, \tau} t_{3}^{\sigma, \tau} \in$ $(0,1)$. In particular, the eigenvalues $E_{\lambda}$ are mutually different for compatible weights when $-\infty<\sigma, \tau<\infty$ (see $\S 3$ ).

We write $D_{\sigma, \tau}$ for Koornwinder's second-order $q^{2}$-difference operator in base $q^{2}$ with parameters $(\underline{t}, t)=\left(\underline{t}^{\sigma, \tau}, q^{2}\right)$, and we write $E_{\mu}^{\sigma, \tau}\left(\mu \in P_{\Sigma}^{+}\right)$for the corresponding eigenvalues. We furthermore write $P_{\mu}^{\sigma, \tau}(x):=P_{\mu}{ }^{K}\left(x ; \underline{t}^{\sigma, \tau} ; q^{2}, q^{2}\right)(\mu \in$ $P_{\Sigma}^{+}$) for the corresponding monic Koornwinder polynomials. By Theorem 7.4, $\varphi^{\sigma, \tau}(\lambda)_{\mid \mathrm{T}} \in \mathbb{C}\left[x^{ \pm 1}\right]$ is an eigenfunction of $D_{\sigma, \tau}$ with eigenvalue $E_{\lambda^{\natural}}^{\sigma, \tau}$ for $\lambda \in \boldsymbol{P}_{K}^{+}$. By [NS1, Lemma 6.2], any eigenfunction $\varphi(x) \in \mathbb{C}\left[x^{ \pm 1}\right]$ of $D_{\sigma, \tau}$ with eigenvalue $E_{\mu}^{\sigma, \tau}\left(\mu \in P_{\Sigma}^{+}\right)$and which is of the particular form

$$
\varphi(x)=c_{\mu} m_{\mu}(x)+\sum_{\nu \in C(\mu)} c_{\nu} x^{\nu}
$$

is a constant multiple of the Koornwinder polynomial $P_{\mu}^{\sigma, \tau}(x)$. Combined with Lemma 7.2, the following main result of the paper [NDS] is obtained.

Theorem 7.5. ([NDS]) Let $-\infty<\sigma, \tau<\infty$. The restriction $\varphi^{\sigma, \tau}(\lambda){ }_{\mid \mathrm{T}}$ of the zonal spherical function $\varphi^{\sigma, \tau}(\lambda) \in \mathscr{H}^{\sigma, \tau}(\lambda)\left(\lambda \in P_{K}^{+}\right)$is equal to the Koornwinder polynomial $P_{\lambda^{\natural}}^{\sigma \tau}(x)$, up to a non-zero scalar multiple. In particular, $\mid \mathrm{T}$ defines an algebra isomorphism from $\mathscr{H}^{\sigma, \tau}$ onto $\mathbb{C}\left[x^{ \pm 1}\right]^{\mathscr{W}}$.

Remark 7.6. It should be observed here that the assumption $-\infty<\sigma, \tau<\infty$ in the preceding arguments is absolutely essential. In fact, the map ${ }_{\mid \mathbb{T}}: A_{q}(U) \rightarrow$ 
$A(\mathbb{T})$ factors through the projection $\pi_{K}: A_{q}(U) \rightarrow A_{q}(K)$. This implies that the image of $\mathscr{H}^{\sigma, \tau}$ under ${ }_{\mid \mathrm{T}}$ is one-dimensional as soon as either $\sigma$ or $\tau$ is infinite.

\section{§8. Limit Transitions on Quantum Grassmannians}

In this section we study the right $\mathfrak{f}^{\tau}$-invariant $(-\infty<\tau \leq \infty)$ functions in the quantized coordinate ring $A_{q}(U / K)=A_{q}\left(\mathfrak{f}^{\infty} \backslash U\right)$. Our method will be to regard this case as a limit of the case $-\infty<\sigma, \tau<\infty$ by sending $\sigma$ to infinity. This limit can be made rigorous by using explicit information about the limit transitions from Koornwinder polynomials to multivariable big and little $q$-Jacobi polynomials. In the rank 1 case these results were derived earlier by Koornwinder [K1] for 2spheres and for arbitrary complex projective space by Noumi and Dijkhuizen [DN1].

For the proper interpretation of the limit transitions of the zonal spherical functions, a careful study is needed of the pre-images of the $\mathscr{W}$-invariant functions $e_{s}(x):=m_{\left(1^{s}\right)}(x)(1 \leq s \leq l)$ under the isomorphism $\mid \mathrm{T}: \mathscr{H}^{\sigma, \tau} \rightarrow \mathbb{C}\left[x^{ \pm 1}\right]^{\mathscr{W}}$. For $-\infty<\sigma, \tau<\infty$, write $e_{r}^{\sigma, \tau}$ for the unique element in $\mathscr{H}^{\sigma, \tau}$ such that its restriction to the torus is equal to $e_{r}(x)(1 \leq r \leq l)$. It is convenient to put $e_{0}(x):=1$ and $e_{0}^{\sigma, \tau}:=1$. Recall that the $\mathscr{W}$-invariant functions $\left\{e_{r}\right\}_{r=1}^{l}$ are algebraically independent generators of $\mathbb{C}\left[x^{ \pm 1}\right]^{\mathscr{W}}$ (cf. $\S 3$ ). In other words, by setting

$$
\hat{P}\left(e_{1}(x), \ldots, e_{l}(x)\right):=P(x), \quad P \in \mathbb{C}\left[x^{ \pm 1}\right]^{\mathscr{W}}
$$

we get an algebra isomorphism $P \longmapsto \hat{P}$ of $\mathbb{C}\left[x^{ \pm 1}\right]^{\mathscr{W}}$ onto $\mathbb{C}[y]$, where $y=\left(y_{1}\right.$, $\left.\ldots, y_{l}\right)$ is an $l$-tuple of independent variables. It follows from Theorem 7.5 that the elements $\left\{e_{r}^{\sigma, \tau}\right\}_{r=1}^{l}$ are algebraically independent generators of the algebra $\mathscr{H}^{\sigma, \tau}$.

Using Theorem 7.5 it is now easy to derive an explicit form of the restriction of the normalized Haar functional $h$ to $\mathscr{H}^{\sigma, \tau}$. Recall that the parameters $\underline{t}^{\sigma, \tau}$ lie in the parameter domain $V_{K}$ for $-\infty<\sigma, \tau<\infty$ (see $\$ 3$ for the definition of $V_{K}$ ). Write $\langle\varphi\rangle_{\sigma, \tau}:=\langle\varphi, 1\rangle_{K, q^{2}, q^{2}}^{\sigma}$ for the constant term of $\varphi \in \mathbb{C}\left[x^{ \pm 1}\right]^{\mathscr{W}}$, with $\langle\cdot, \cdot\rangle_{K}$ defined by (3.1). Observe that $\langle 1\rangle_{\sigma, \tau}$ is non-zero by the positive definiteness of $\langle\cdot, \cdot\rangle_{K}$. Explictly, $\langle 1\rangle_{\sigma, \tau}$ can be given explicitly as product and quotient of $q$ Gamma functions by Gustafson's evaluation of the multidimensional Askey-Wilson integral (3.2).

Corollary 8.1. Let $-\infty<\sigma, \tau<\infty$. The restricted Haar functional $h: \mathscr{H}^{\sigma, \tau} \rightarrow$ $\mathbb{C}$ is explicitly given by

$$
h\left(\hat{P}\left(e_{1}^{\sigma, \tau}, e_{2}^{\sigma, \tau}, \ldots, e_{l}^{\sigma, \tau}\right)\right)=\frac{\langle P\rangle_{\sigma, \tau}}{\langle 1\rangle_{\sigma, \tau}} \quad\left(P \in \mathbb{C}\left[x^{ \pm 1}\right]^{\mathscr{W}}\right) .
$$

Proof. The left- and the right-hand side are both equal to zero for $P=P_{\mu}^{\sigma, \tau}$ 
with $0 \neq \mu \in P_{\Sigma}^{+}$, and both equal to 1 for $P=1$. The corollary follows now by linearity, since the Koornwinder polynomials $P_{\mu}^{\sigma, \tau}(x)\left(\mu \in P_{\Sigma}^{+}\right)$form a linear basis of $\mathbb{C}\left[x^{ \pm 1}\right]^{\mathscr{W}}$.

Recall the intertwiner

$$
\hat{\Psi}_{r}:\left(V \otimes V^{*}\right)^{\otimes r} \rightarrow \Lambda_{q}^{r}(V) \otimes \Lambda_{q}^{r}\left(V^{*}\right)
$$

defined in Proposition 6.11. Introduce left $\mathfrak{f}^{\sigma}$-fixed vectors $w_{r}^{\sigma} \in \Lambda_{q}^{r}(V) \otimes \Lambda_{q}^{r}\left(V^{*}\right)$ and right $\mathfrak{f}^{\tau}$-fixed vectors $\tilde{w}_{r}^{\tau} \in \Lambda_{q}^{r}(V)^{\circ} \otimes \Lambda_{q}^{r}\left(V^{*}\right)^{\circ}$ by

$$
w_{r}^{\sigma}:=\hat{\Psi}_{r}\left(\left(w^{\sigma}\right)^{\otimes r}\right), \quad \tilde{w}_{r}^{\tau}:=\hat{\Psi}_{r}\left(\left(\tilde{w}^{\tau}\right)^{\otimes r}\right) \quad(1 \leq r \leq l) .
$$

Here we have used the notation $\tilde{w}^{\infty}:=w^{\infty}(6.11)$ when $\tau=\infty$, which is consistent with the definition of $\tilde{w}^{\tau}$ for $-\infty<\tau<\infty$ since $\lim _{\tau \rightarrow \infty} \tilde{w}^{\tau}=w^{\infty}$. Consider now the $(\sigma, \tau)$-spherical elements

$$
\varphi_{r}^{\sigma, \tau}:=\theta\left(\tilde{w}_{r}^{\tau}, w_{r}^{\sigma}\right) \in \bigoplus_{s=0}^{r} \mathscr{H}^{\sigma, \tau}\left(\varpi_{s}\right) \quad(1 \leq r \leq l)
$$

(cf. (6.14)), where $\theta$ is the map (7.2) associated with the unitary comodule $\Lambda_{q}^{r}(V)$ $\otimes \Lambda_{q}^{r}\left(V^{*}\right)$ endowed with the inner product $\langle\cdot, \cdot\rangle$ (see (7.6) for the definition of $\langle\cdot, \cdot\rangle)$. It is convenient to put $\varphi_{0}^{\sigma, \tau}:=1$. By Theorem 7.5 and $(8.2),\left.\varphi_{r}^{\sigma, \tau}\right|_{\mathrm{T}}$ is a linear combination of the $\mathscr{W}$-invariant functions $e_{s}(x) \in \mathbb{C}\left[x^{ \pm 1}\right]^{\mathscr{W}}(0 \leq s \leq r)$.

Lemma 8.2. Let $-\infty<\sigma, \tau<\infty, 1 \leq r \leq l$. In the expansion

$$
\varphi_{r}^{\sigma, \tau}{ }_{\mid \mathbb{T}}=a_{r}^{r}\left(q^{\sigma}, q^{\tau}\right) e_{r}+\cdots+a_{0}^{r}\left(q^{\sigma}, q^{\tau}\right) e_{0}
$$

each coefficient $a_{i}^{r}$ is a polynomial in $q^{\sigma}$ and $q^{\tau}$ which is the sum of monomials of partial degree $\geq i$ in each of the variables. Moreover, $a_{r}^{r}\left(q^{\sigma}, q^{\tau}\right)=c q^{r \sigma+r \tau}$ with $c \neq 0$ independent of $q^{\sigma}$ and $q^{\tau}$.

Proof. It is obvious from the definitions that the coefficients are polynomial in $q^{\sigma}$ and $q^{\tau}$. To prove the estimates on the partial degrees, we study the action of the intertwiner $\hat{\Psi}_{r}$ on the vectors $\left(w^{\sigma}\right)^{\otimes r}$ and $\left(\tilde{w}^{\tau}\right)^{\otimes r}$ in detail. We proceed in a number of steps.

(1) Let $1 \leq i_{1} \leq \cdots \leq i_{r} \leq n$ and $1 \leq j_{1} \leq \cdots \leq j_{r} \leq n$ be integers. We use the shorthand notation $\underline{i}:=\left(i_{1}, \ldots, i_{r}\right), \underline{j}:=\left(j_{1}, \ldots, j_{r}\right)$. Call a tensor $t$ in some tensor product space made up of factors $V$ or $V^{*}$ (the total number of factors $V$ being equal to the total number of factors $\left.V^{*}\right)$ a basic tensor of type $(\underline{i}, \underline{j})$ if $t$ is the tensor product in any given order of the vectors $v_{i_{1}}, \ldots, v_{i_{r}}$ and $v_{j_{1}}^{*}, \ldots, v_{j_{r}}^{*}$. Let $n_{k}(\underline{i})$ denote the cardinality of the set $\left\{p \in[1, r] \mid i_{p}=k\right\}$. For a basic tensor $t$ of type 
$(\underline{i}, \underline{j})$ define

$$
n(t):=\sum_{k=1}^{n} \min \left(n_{k}(\underline{i}), n_{k}(\underline{j})\right) .
$$

From an informal point of view, $n(t)$ is the number of factors $v_{i}$ in $t$ that "cancel" against a factor $v_{i}^{*}$. Recall the intertwiner $\Psi_{r}:\left(V \otimes V^{*}\right)^{\otimes r} \rightarrow V^{\otimes r} \otimes\left(V^{*}\right)^{\otimes r}$ defined in (6.20). Let $t$ be a basic tensor in $\left(V \otimes V^{*}\right)^{\otimes r}$. Since $\Psi_{r}$ is a composition of intertwiners $\beta_{i j}$ (see (6.20)) it follows by inspection of (6.19) that $\Psi_{r}(t)$ is a linear combination of basic tensors $t^{\prime}$ in $V^{\otimes r} \otimes\left(V^{*}\right)^{\otimes r}$ with $n\left(t^{\prime}\right)=n(t)$.

(2) A basic tensor $t \in\left(V \otimes V^{*}\right)^{\otimes r}$ is called typical if it is a product of tensors in $V \otimes V^{*}$ of type $v_{i} \otimes v_{i}^{*}(1 \leq i \leq n-l), v_{i} \otimes v_{i^{*}}^{*}, v_{i^{\prime}} \otimes v_{i}^{*}(1 \leq i \leq l)$. We call a typical tensor $t \in\left(V \otimes V^{*}\right)^{\otimes r} k$-typical if the number of factors of type $v_{i} \otimes v_{i}{ }^{*}(i \in[1, l] \cup$ $\left.\left[l^{\prime}, n\right]\right)$ is equal to $k$. If $t$ is a $k$-typical tensor then $\hat{\Psi}_{r}(t)$ is a linear combination of elements $v_{I} \otimes v_{J}^{*}$ where $I, J \subset[1, n]$ are such that $|I|=|J|=r$ and $|I \cap J| \geq r-k$. In fact, this follows from (1) and the definition of $\hat{\Psi}_{r}$, since $n(t) \geq r-k$.

(3) It is an immediate consequence of the definition of the coactions on $\Lambda_{q}^{r}(V)$ and $\Lambda_{q}^{r}\left(V^{*}\right)$ and of (4.11) that

$$
\theta\left(v_{I} \otimes v_{J}^{*}, v_{K} \otimes v_{L}^{*}\right)_{\mid T}=q^{-\left\langle 2 \rho, \tilde{\varepsilon}_{J}\right\rangle} \delta_{I, K} \delta_{J, L} z^{\tilde{\varepsilon}_{I}-\tilde{\varepsilon}_{J}},
$$

for $I, J \subset[1, n]$ with $|I|=|J|=r$.

(4) Let $t$ be a $k$-typical tensor and $t^{\prime}$ an $m$-typical tensor. Let $\mu \in \mathscr{W}\left(1^{i}\right) \subset P_{\Sigma}$ $(i \in[1, r])$ be any weight, and suppose that the coefficient of $z^{\mu^{b}}$ in the expansion of $\theta\left(\hat{\Psi}_{r}(t), \hat{\Psi}_{r}\left(t^{\prime}\right)\right)_{i \mathbb{T}}$ with respect to the basis $\left\{z^{\lambda}\right\}_{\lambda \in P}$ of $\boldsymbol{A}(\mathbb{T})$, is non-zero. Then $k \geq i$ and $m \geq i$. This is a straightforward consequence of (2) and (3).

(5) There is a unique expansion $\left(w^{\sigma}\right)^{\otimes r}=\sum_{k=0}^{r} \sum_{t_{k}} c_{t_{k}} t_{k}$ where $t_{k}$ runs over all $k$-typical tensors in $\left(V \otimes V^{*}\right)^{\otimes r}$. The non-zero $c_{t_{k}}$ are linear combinations of monomials $\left(q^{\sigma}\right)^{i}$ with $i \geq k$. Similarly, there is a unique expansion $\left(\tilde{\boldsymbol{w}}^{\tau}\right)^{\otimes r}=\sum_{k=0}^{r}$ $\sum_{t^{\prime}{ }_{k}} d_{t^{\prime}} t_{k}^{\prime}$ where $t_{k}$ runs over all $k$-typical tensors in $\left(V \otimes V^{*}\right)^{\otimes r}$. The non-zero $d_{t^{\prime}}$ are linear combinations of monomials $\left(q^{\tau}\right)^{i}$ with $i \geq k$. Hence,

$$
\varphi_{r}^{\sigma, \tau}{ }_{\mid \mathbb{T}}=\sum_{k, m=0}^{r} \sum_{t_{k}, t_{m}} c_{t_{k} t_{m}^{\prime}} \theta\left(\hat{\Psi}_{r}\left(t_{m}^{\prime}\right), \hat{\Psi}_{r}\left(t_{k}\right)\right)_{\mid \mathbb{T}}
$$

with $c_{t_{k} t_{m}^{\prime}}=c_{t_{k}} \overline{d_{t^{\prime}}}$ a linear combination of monomials $\left(q^{\sigma}\right)^{i}\left(q^{\tau}\right)^{j}$ with $i \geq k$ and $j \geq m$. Combined with (4) this yields the desired lower bounds on the partial degrees of the monomials $\left(q^{\sigma}\right)^{i}\left(q^{\tau}\right)^{j}$ occurring in $a_{i}^{r}\left(q^{\sigma}, q^{\tau}\right)$. An explicit expression for $a_{r}^{r}\left(q^{\sigma}\right.$, $q^{\tau}$ ) can be given using Proposition 6.11. The last statement of the proposition follows then immediately.

As a corollary we obtain the following crucial lemma.

Lemma 8.3. Let $-\infty<\tau<\infty$. The limits 


$$
\lim _{\sigma \rightarrow \infty} q^{r \sigma} e_{r}^{\sigma, \tau}, \quad \lim _{\sigma \rightarrow \infty} q^{2 r \sigma} e_{r}^{\sigma, \sigma} \quad(1 \leq r \leq l)
$$

exist in $A_{q}(U)$. In other words, the coefficients of $q^{r \sigma} e_{r}^{\sigma, \tau}$ respectively $q^{2 r \sigma} e_{r}^{\sigma, \sigma}$ in the expansion with respect to the monomial basis of $A_{q}(U)$ tend to finite values in the limit $\sigma \rightarrow \infty$.

Proof. Fix $1 \leq r \leq l$ and let $-\infty<\sigma, \tau<\infty$. From Lemma 8.2 it is readily deduced that

$$
q^{r \sigma+r \tau} e_{r}=b_{\tau}^{r}\left(q^{\sigma}, q^{\tau}\right) \varphi_{r}^{\sigma, \tau}\left|\mathbb{T}+\cdots+b_{0}^{r}\left(q^{\sigma}, q^{\tau}\right) \varphi_{0}^{\sigma, \tau}\right| \mathrm{T}
$$

with $b_{i}^{r}(0 \leq i \leq r)$ some polynomial in two variables and $b_{r}^{r}$ a non-zero constant polynomial (the important fact here is that $b_{i}^{r}$ is a polynomial and not a Laurent polynomial). Hence

$$
e_{r}^{\sigma, \tau}=q^{-r \sigma-r \tau}\left(b_{r}^{r}\left(q^{\sigma}, q^{\tau}\right) \varphi_{r}^{\sigma, \tau}+\cdots+b_{0}^{r}\left(q^{\sigma}, q^{\tau}\right) \varphi_{0}^{\sigma, \tau}\right)
$$

Since $\varphi_{i}^{\sigma, \tau} \rightarrow \varphi_{i}^{\infty, \tau}$ and $\varphi_{i}^{\sigma, \sigma} \rightarrow \varphi_{i}^{\infty, \infty}$ when $\sigma \rightarrow \infty$, the lemma follows.

In view of Lemma 8.3 we may set for $1 \leq r \leq l$ and $-\infty<\tau<\infty$,

$$
\tilde{e}_{r}^{\infty, \tau}:=\lim _{\sigma \rightarrow \infty} q^{r(\sigma+\tau-1)}(-1)^{r} e_{r}^{\sigma, \tau}, \quad \tilde{e}_{r}^{\infty, \infty}:=\lim _{\sigma \rightarrow \infty} q^{r(2 \sigma-1)}(-1)^{r} e_{r}^{\sigma, \sigma} .
$$

It is clear from the definitions that $\tilde{e}_{r}^{\infty, \tau} \in \mathscr{H}^{\infty, \tau}(1 \leq r \leq l,-\infty<\tau \leq \infty)$. Observe that the elements $(-1)^{r} e_{r}^{\sigma, \tau}$ are mapped onto $e_{r}(-x) \in \mathbb{C}\left[x^{ \pm 1}\right]^{\mathscr{W}}$ under the restriction mapping $\mid \mathrm{T}$.

Recall that the elements $\tilde{e}_{r}(1 \leq r \leq l)$ are algebraically independent generators of the algebra $\mathbb{C}[x]^{\mathcal{\odot}}$ (cf. §3). Again, we obtain an algebra isomorphism $P \rightarrow \hat{P}$ of $\mathbb{C}[x]^{\subseteq}$ onto $\mathbb{C}[y]$, where

$$
\hat{P}\left(\tilde{e}_{1}(x), \ldots, \tilde{e}_{l}(x)\right):=P(x), \quad\left(P \in \mathbb{C}[x]^{\varpi}\right) .
$$

Theorem 8.4. Let $-\infty<\tau<\infty$. The elements $\tilde{e}_{r}^{\infty, \tau}(1 \leq \boldsymbol{r} \leq l)$ mutually commute and are algebraically independent generators of the algebra $\mathscr{H}^{\infty} \tau$. Any zonal $(\infty, \tau)$-spherical function $\varphi^{\infty, \tau}(\lambda) \in \mathscr{H}^{\infty}, \tau(\lambda)\left(\lambda \in P_{K}^{+}\right)$is equal to a non-zero scalar multiple of

$$
\hat{P}_{\lambda^{\natural}}^{B}\left(\tilde{e}_{1}^{\infty, \tau}, \ldots, \tilde{e}_{l}^{\infty, \tau} ; 1, q^{2(n-2 l)}, 1, q^{2 \tau+2(n-2 l)} ; q^{2}, q^{2}\right),
$$

where $P_{\mu}^{B}(. ; a, b, c, d ; q, t)$ is the multivariable big $q$-Jacobi polynomial of degree $\mu$ (cf. §3). 
Proof. The elements $e_{r}^{\sigma, \tau}(1 \leq r \leq l)$ mutually commute for $\sigma$ finite by Corollary 7.3. By the definition of the elements $\tilde{e}_{r}^{\infty, \tau}(8.4)$, it follows that the $\tilde{e}_{r}^{\infty}, \tau$ $(1 \leq r \leq l)$ also commute. Hence the element $Q\left(\tilde{e}_{1}^{\infty, \tau}, \ldots, \tilde{e}_{l}^{\infty, \tau}\right) \in \mathscr{H}^{\infty, \tau}$ for a polynomial $Q \in \mathbb{C}[y]$ is well defined.

For $\sigma$ finite and $\lambda \in P_{K}^{+}$, a zonal $(\sigma, \tau)$-spherical function $\varphi^{\sigma, \tau}(\lambda) \in \mathscr{H}^{\sigma, \tau}(\lambda)$ is given by

$$
\varphi^{\sigma, \tau}(\lambda):=\left(-q^{\sigma+\tau-1}\right)^{\left|\lambda^{\natural}\right|} \hat{P}_{\lambda^{\natural}}^{K}\left(e_{1}^{\sigma, \tau}, \ldots, e_{l}^{\sigma, \tau} ; \underline{t}^{\sigma, \tau} ; q^{2}, q^{2}\right)
$$

with $P_{\mu}^{K}$ (.) the Koornwinder polynomial of degree $\mu$ (cf. Theorem 7.5). Using the elementary properties of the Koornwinder polynomials given in Lemma 3.1, this zonal $(\sigma, \tau)$-spherical function can be rewritten as

$$
\varphi^{\sigma, \tau}(\lambda)=\left(s_{\varepsilon}\right)^{-\left|\lambda^{\natural}\right|} \hat{P}_{\lambda^{\natural}}^{K}\left(s_{\varepsilon} f_{1}^{\varepsilon}, \ldots, s_{\varepsilon}^{l} f_{l}^{\varepsilon} ; \underline{t}_{B}(\varepsilon) ; q^{2}, q^{2}\right),
$$

where $s_{\varepsilon}:=q / \varepsilon(c d)^{\frac{1}{2}}, f_{r}^{\varepsilon}:=\left(-s_{\varepsilon}\right)^{-r} e_{r}^{\sigma, \tau}, \varepsilon:=q^{\sigma-(n-2 l)}$, and

$$
\underline{t}_{B}(\varepsilon)=\left(\varepsilon^{-1}\left(q^{2} c / d\right)^{\frac{1}{2}},-\varepsilon^{-1}\left(q^{2} d / c\right)^{\frac{1}{2}}, \varepsilon a\left(q^{2} d / c\right)^{\frac{1}{2}},-\varepsilon b\left(q^{2} c / d\right)^{\frac{1}{2}}\right),
$$

with parameters $a, b, c, d$ given by

$$
a:=1, \quad b:=q^{2(n-2 l)}, \quad c:=1, \quad d:=q^{2 \tau+2(n-2 l)} .
$$

Observe that $s_{\varepsilon}=q^{1-\sigma-\tau}$, hence by the definition (8.4) of $\tilde{e}_{r}^{\infty, \tau}, \lim _{\varepsilon \downarrow 0} f_{r}^{\varepsilon}=\tilde{e}_{r}^{\infty, \tau}$ for all $r$. Combined with the limit transition from Koornwinder polynomials to multivariable big $q$-Jacobi polynomials (3.19), we get that $\varphi^{\infty, \tau}(\lambda):=\lim _{\sigma \rightarrow \infty} \varphi^{\sigma, \tau}(\lambda)$ exists as limit in $A_{q}(U)$, and that

$$
\varphi^{\infty, \tau}(\lambda)=\hat{P}_{\lambda^{\natural}}^{B}\left(\tilde{e}_{1}^{\infty, \tau}, \ldots, \tilde{e}_{l}^{\infty, \tau} ; 1, q^{2(n-2 l)}, 1, q^{2 \tau+2(n-2 l)} ; q^{2}, q^{2}\right)
$$

with $P_{\mu}^{B}$ (.) the multivariable big $q$-Jacobi polynomial of degree $\mu$. It is clear that $\varphi^{\infty, \tau}(\lambda) \in \mathscr{H}^{\infty, \tau}(\lambda)$, but it may be zero since the algebraic independence of the elements $\tilde{e}_{r}^{\infty, \tau}(r \in[1, l])$ has not yet been established. To prove that $\varphi^{\infty, \tau}(\lambda)$ is non-zero, we compute the quadratic norm $\left\|\varphi^{\sigma, \tau}(\lambda)\right\|^{2}$ with respect to the inner product $\langle\varphi, \phi\rangle:=h\left(\phi^{*} \varphi\right)$, where $h$ is the normalized Haar functional. Since all highest weights $\lambda \in P_{K}^{+}$are self-dual (i.e. $V(\lambda)$ is isomorphic to its dual representation), and since the two-sided coideal $\mathfrak{f}^{\sigma}$ is $\tau$-invariant, we have $\left(\varphi^{\sigma, \tau}(\lambda)\right)^{*}=$ $\varphi^{\sigma, \tau}(\lambda)$. Then it follows from the definition (8.6) of $\varphi^{\sigma, \tau}(\lambda)$, Corollary 8.1 and the definition (3.21) of $N_{K}$, that

$$
\left\|\varphi^{\sigma, \tau}(\lambda)\right\|^{2}=s_{\varepsilon}^{-2\left|\lambda^{\natural}\right|} N_{K}\left(\lambda^{\natural} ; \underline{t}_{B}(\varepsilon) ; q^{2}, q^{2}\right) .
$$


The limit $\varepsilon \downarrow 0$ (equivalently, $\sigma \rightarrow \infty$ ) in (8.9) can now be computed in the lefthand side and in the right-hand side (cf. Proposition 3.2). It follows that

$$
\left\|\varphi^{\infty, \tau}(\lambda)\right\|^{2}=N_{B}\left(\lambda^{\natural} ; 1, q^{2(n-2 l)}, 1, q^{2 \tau+2(n-2 l)} ; q^{2}, q^{2}\right) .
$$

Since $N_{B}\left(\lambda^{\natural}\right)$ is strictly positive, it follows that the quadratic norm $\left\|\varphi^{\infty, \tau}(\lambda)\right\|^{2}$ is non-zero, hence $\varphi^{\infty, \tau}(\lambda) \neq 0$ for all $\lambda \in P_{K}^{+}$. Hence the elements $\varphi^{\infty, \tau}(\lambda) \in \mathscr{H}^{\infty, \tau}(\lambda)$ are zonal $(\infty, \tau)$-spherical functions for all $\lambda \in P_{K}^{+}$.

It remains to prove that the $\tilde{e}_{r}^{\infty, \tau}(1 \leq r \leq l)$ are algebraically independent. Consider the finite-dimensional subspaces

$$
\mathscr{H}_{m}:=\bigoplus_{\lambda \in P_{x}^{*}} \bigoplus_{\lambda \leq m \sigma_{l}} \mathscr{H}^{\infty, \tau}(\lambda), \quad\left(m \in \mathbb{Z}_{+}\right) .
$$

The dimension of the linear subspace $\mathscr{H}_{m}$ is equal to the number of positive integers $\underline{m}=\left(m_{1}, \ldots, m_{l}\right) \in \mathbb{Z}_{+}^{\times l}$ with $|\underline{m}|:=\sum_{i} m_{i} \leq m$, since $\varpi_{r} \leq \varpi_{l}$ for all $r \in[0, l]$. For such a sequence of positive integers $\underline{m}$, set $Q_{m}(y):=y_{1}^{m_{1}} \ldots y_{l}^{m_{l}}$. Since $\tilde{e}_{r}^{\infty}, \epsilon$ $\oplus_{s=0}^{r} \mathscr{H}^{\infty, \tau}\left(\varpi_{s}\right)$ and

$$
\mathscr{H}^{\infty, \tau}(\mu) . \mathscr{H}^{\infty, \tau}\left(\mu^{\prime}\right) \subseteq \bigoplus_{\nu \in P_{R^{+}}^{+}} \bigoplus_{\nu \leq \mu+\mu^{\prime}} \mathscr{H}^{\infty, \tau}(\nu), \quad\left(\mu, \mu^{\prime} \in P_{K}^{+}\right)
$$

we have $Q_{\underline{m}}\left(\tilde{e}_{1}^{\infty, \tau}, \ldots, \tilde{e}_{l}^{\infty, \tau}\right) \in \mathscr{H}_{m}$ for all $\underline{m}$ with $|\underline{m}| \leq m$. Hence the algebraic independence of $\tilde{e}_{r}^{\infty} \tau(1 \leq r \leq l)$ will follow from the fact that the monomials $Q_{\underline{m}}\left(\tilde{e}_{1}^{\infty}, \tau, \ldots, e_{l}^{\infty, \tau}\right)(|\underline{m}| \leq m)$ span $\mathscr{H}_{m}$ for all $m \in \mathbb{Z}_{+}$.

Observe that $\mathscr{H}_{m}$ is spanned by the zonal spherical functions $\varphi^{\infty, \tau}(\lambda)(\lambda \leq$

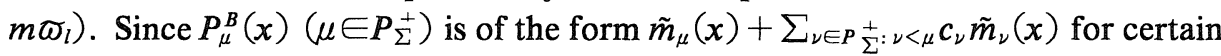
constants $c_{\nu}$, it follows from the explicit expression (8.8) for $\varphi^{\infty, \tau}(\lambda)$ that each $\varphi^{\infty, \tau}(\lambda)$ with $\lambda \leq m \varpi_{l}$ can be written as a linear combination of the monomials $Q_{\underline{m}}\left(\tilde{e}_{1}^{\infty, \tau}, \ldots, \tilde{e}_{l}^{\infty, \tau}\right)(|\underline{m}| \leq m)$. Hence, the monomials $Q_{\underline{m}}\left(\tilde{e}_{1}^{\infty}, \tau, \ldots, \tilde{e}_{l}^{\infty, \tau}\right)(|\underline{m}| \leq$ m) $\operatorname{span} \mathscr{H}_{m}$.

Theorem 8.5. The elements $\tilde{e}_{r}^{\infty, \infty}(1 \leq r \leq l)$ mutually commute and are algebraically independent generators of the algebra $\mathscr{H}^{\infty, \infty}$. Any zonal spherical function $\varphi^{\infty, \infty}(\lambda)\left(\lambda \in P_{K}^{+}\right)$is equal to a non-zero scalar multiple of

$$
\hat{P}_{\lambda^{\natural}}^{L}\left(\tilde{e}_{1}^{\infty, \infty}, \ldots, \tilde{e}_{l}^{\infty, \infty} ; q^{2(n-2 l)}, 1 ; q^{2}, q^{2}\right)
$$

where $P_{\mu}^{L}($.$) is the multivariable little q$-Jacobi polynomial of degree $\mu$ (cf. §3).

Proof. By Theorem 7.5 and by symmetry properties of the Koornwinder polynomials (cf. Lemma 3.1), a zonal $(\sigma, \sigma)$-spherical function $\varphi^{\sigma, \sigma}(\lambda) \in \mathscr{H}^{\sigma, \sigma}(\lambda)$ $\left(\lambda \in P_{K}^{+}\right)$is explicitly given by 


$$
\varphi^{\sigma, \sigma}(\lambda):=\left(s_{\varepsilon}\right)^{-\left|\lambda^{\natural}\right|} \hat{P}_{\lambda^{\natural}}^{K}\left(s_{\varepsilon} f_{1}^{\varepsilon}, \ldots, s_{\varepsilon}^{l} f_{l}^{\varepsilon} ; \underline{t}_{L}(\varepsilon) ; q^{2}, q^{2}\right),
$$

where $P_{\mu}^{K}($.$) is the Koornwinder polynomial of degree \mu$ (cf. $\S 3$ ) and $\varepsilon:=q^{2 \sigma}$, $s_{\varepsilon}:=q / \varepsilon, f_{r}^{\varepsilon}:=\left(-s_{\varepsilon}\right)^{-r} e_{r}^{\sigma, \sigma}$, and $\underline{t}_{L}(\varepsilon):=\left(\varepsilon^{-1} q,-a q, \varepsilon b q,-q\right)$ with $a:=q^{2(n-2 l)}$ and $b:=1$. Using (3.20) together with the observation that $\lim _{\varepsilon \downarrow 0} f_{r}^{\varepsilon}=\tilde{e}_{r}^{\infty}, \infty$, the proof is analogous to the proof of Theorem 8.4.

Remark 8.6. Using the limits $\tilde{e}_{r}^{\infty, \infty}=\lim _{\tau \rightarrow \infty} \tilde{e}_{r}^{\infty, \tau}(1 \leq r \leq l)$, Theorem 8.5 can also be proved by sending $\tau \rightarrow \infty$ in the results of Theorem 8.4. On the level of multivariable orthogonal polynomials this limit corresponds to the limit from multivariable big $q$-Jacobi polynomials to multivariable little $q$-Jacobi polynomials proved in [SK, Thm. 5.1(3)].

Remark 8.7. As a corollary of Theorem 8.4 and Theorem 8.5, the restricted Haar functional $\boldsymbol{h}: \mathscr{H}^{\sigma, \tau} \rightarrow \mathbb{C}$ for $\sigma=\infty,-\infty<\tau<\infty$ respectively for $\sigma=\tau=\infty$ can be expressed in terms of the orthogonality measure of the multivariable big respectively little $q$-Jacobi polynomials (cf. Corollary 8.1).

Remark 8.8. In the last two sections we have interpreted the Koornwinder polynomial resp. the multivariable big and the multivariable little $q$-Jacobi polynomial

$$
\begin{aligned}
& P_{\mu}^{K}\left(. ;-q^{\sigma+\tau+1},-q^{-\sigma-\tau+1}, q^{\sigma-\tau+1}, q^{-\sigma+\tau+2(n-2 l)+1} ; q^{2}, q^{2}\right) \\
& P_{\mu}^{B}\left(. ; 1, q^{2(n-2 l)}, 1, q^{2 \tau+2(n-2 l)} ; q^{2}, q^{2}\right) \\
& P_{\mu}^{L}\left(. ; q^{2(n-2 l)}, 1 ; q^{2}, q^{2}\right)
\end{aligned}
$$

$\left(-\infty<\sigma, \tau<\infty, \mu \in P_{K}^{+}\right)$as a zonal spherical function on some quantum analogue of the complex Grassmannian. For each of these polynomials, the classical limit $q \uparrow 1$ can be computed using results from [Dj1, §4] for the Koornwinder polynomials and using $[\mathrm{SK}$, Thm. 5.1(5) \& (6)] for the multivariable big and little $q$-Jacobi polynomials. The limits can be written in terms of the generalized Jacobi polynomial $P_{\mu}^{J}(. ; n-2 l, 0 ; 1)$ or, equivalently, in terms of the $B C$ type HeckmanOpdam polynomial $P_{\mu}^{H O}(. ; n-2 l, 1,1 / 2)$. This agrees nicely with the classical interpretation of the Heckman-Opdam polynomial $P_{\mu}^{H O}(. ; n-2 l, 1,1 / 2)$ as a zonal spherical function on the complex Grassmannian $U / K$ (see §2).

\section{Acknowledgements}

The authors would like to thank Prof. Tom H. Koornwinder and Prof. Masatoshi Noumi for interesting and informative discussions about the topic of this 
paper.

\section{References}

[A] Askey, R., Some basic hypergeometric extensions of integrals of Selberg and Andrews, SIAM J. Math. Anal., $\mathbb{1 1}$ (1980), no. 6, 938-951.

[AW] Askey, R., Wilson, J., Some basic hypergeometric orthogonal polynomials that generalize Jacobi polynomials, Mem. Amer. Math. Soc., 54 (1985), no. 319, 1-55.

[B] Bourbaki, N., Groupes et Algèbres de Lie, Ch. 4, 5, 6, Masson, Paris, 1981.

[CP] Chari, V., Pressley, A., A Guide to Quantum Groups, Cambridge University Press, Cambridge, UK, 1994.

[Dj1] van Diejen, J. F., Commuting difference operators with polynomial eigenfunctions, Compositio Math., 95 (1995), 183-233.

[Dj2] _ Self-dual Koornwinder-Macdonald polynomials, Invent. Math., 126 (1996), 319-339.

[Dz] Dijkhuizen, M. S., Covariant Poisson brackets, quantized complex Grassmannians, and $B C$ type $q$-orthogonal polynomials, in : Quantum Group Symposium at Group21, (Proceedings of the Quantum Group Symposium at the XXI International Colloquium on Group Theoretical Methods in Physics, Goslar, 1996), eds. H. -D. Doebner, V. K. Dobrev, pp. 345-353, Heron Press, Sofia (1997).

[DK1] Dijkhuizen, M.S., Koornwinder, T. H., Quantum homogeneous spaces, duality and quantum 2-spheres, Geom. Dedicata, 52 (1994), 291-315.

[DK2] , CQG algebras : a direct algebraic approach to compact quantum groups, Lett. Math. Phys., 32 (1994), 315-330.

[DN1] Dijkhuizen, M. S., Noumi, M., A family of quantum projective spaces and related $q$ hypergeometric orthogonal polynomials, Trans. Amer. Math. Soc., 350 (1998), 3269-3296.

[DG] Donin, J., Gurevich, D., Some Poisson structures associated to Drinfeld-Jimbo R-matrices and their quantization, Israel Math. J., 92 (1995), 23-32.

[Dr] Drinfel'd, V. G., Quantum groups, in : Proceedings International Conference of Mathematicians, Berkeley, 1986, ed. A. M. Gleason, Amer. Math. Soc., Providence, RI (1987), 798-820.

[E] Evans, R. J., Multidimensional beta and gamma integrals, in : The Rademacher Legacy to Mathematics, eds. G. E. Andrews e.a., Contemp. Math., 166 (1994), 341-357.

[G] Gustafson, R. A., A generalization of Selberg's beta integral, Bull. Amer. Math. Soc. (N. S.), 22 (1990), no. 1, 97-105.

[Ha] Habsieger, L., Une $q$-intégrale de Selberg et Askey, SIAM J. Math. Anal., 19 (1988), 14751489.

[HH] Hashimoto, M., Hayashi, T., Quantum multilinear algebra, Tôhoku Math. J., 44 (1992), 471-521.

[HS] Heckman, G. J., Schlichtkrull, H., Harmonic Analysis and Special Functions on Symmetric Spaces, Perspectives in Math. 16, Academic Press, New York, 1995.

[He] Helgason, S., Groups and Geometric Analysis, Academic Press, New York, 1984.

[J] Jimbo, M., A $q$-analogue of $U(\mathrm{gl}(n))$, Hecke algebra and the Yang-Baxter equation, Lett. Math. Phys., 11 (1986), 247-252.

$[\mathrm{Ka}] \quad$ Kadell, K. W. J., A proof of Askey's conjectured $q$-analogue of Selberg's integral and a conjecture of Morris, SIAM J. Math. Anal., 19 (1988), 969-986.

[KRR] Khoroshkin, S., Radul, A., Rubtsov, V., A family of Poisson structures on Hermitian 
symmetric spaces, Comm. Math. Phys., 152 (1993), 299-315.

[KS] Klimyk, A., Schmüdgen, K., Quantum Groups and Their Representations, Springer-Verlag, Berlin, 1997.

[K1] Koornwinder, T. H., Representations of the twisted $S U$ (2) quantum group and some $q$ hypergeometric polynomials, Proc. Kon. Ned. Akad. Wet. Series A, 92 (1989), 97-117.

[K2] Orthogonal polynomials in connection with quantum groups, in Orthogonal polynomials : Theory and Practice, NATO ASI Ser. C, 294 (1990), 257-292.

[K3] Askey-Wilson polynomials for root systems of type $B C$, in : Hypergeometric functions on domains of positivity, Jack polynomials, and applications, ed. D.S.P. Richards, Contemp. Math., 138 (1992), 189-204.

[K4] Askey-Wilson polynomials as zonal spherical functions on the $S U(2)$ quantum group, SIAM J. Math. Anal., 24 (1993), no. 3, 795-813.

[M1] Macdonald, I. G., Orthogonal polynomials associated with root systems, unpublished manuscript (1988).

[M2] _ Symmetric Functions and Hall Polynomials, 2nd ed., Oxford University Press, Oxford, 1995.

¿Ms] Masuda, T., Mimachi, K., Nakagami, Y., Noumi, M., Ueno, K., Representations of quantum groups and a $q$-analogue of orthogonal polynomials, C. $R$. Acad. Sci. Paris Sér. I Math., 307 (1988), 559-564.

[N] Noumi, M., Macdonald's symmetric polynomials as zonal spherical functions on some quantum homogeneous spaces, Adv. Math., 123 (1996), no. 1, 16-77.

[NDS] Noumi, M., Dijkhuizen, M. S., Sugitani, T., Multivariable Askey-Wilson polynomials and quantum complex Grassmannians, in : Special Functions, $q$-Series, and Related Topics, eds. M. E. H. Ismail e.a., Fields Inst. Comm., 14 (1997), 167-177.

[NM] Noumi, M., Mimachi, K., Quantum 2-spheres and big $q$-Jacobi polynomials, Comm. Math. Phys., 128 (1990), 521-531.

[NS1] Noumi, M., Sugitani, T., Quantum symmetric spaces and related $q$-orthogonal polynomials, in : Group Theoretical Methods in Physics, Proceedings XX ICGTMP, Toyonaka (Japan), July 1994, eds. A. Arima et al., pp. 28-40, World Scientific, Singapore, 1995.

[NS2] _ Quantum symmetric spaces and multivariable $q$-orthogonal polynomials, preprint (1996).

[NYM] Noumi, M., Yamada, H., Mimachi, K., Finite-dimensional representations of the quantum group $G L_{q}(n, \mathbb{C})$ and the zonal spherical functions on $U_{q}(n-1) \backslash U_{q}(n)$, Japan. J. Math., 19 (1993), no. 1, 31-80.

[P] Podles̀, P., Quantum spheres, Lett. Math. Phys., 14 (1987), 193-202.

[RTF] Reshetikhin, N., Takhtadjan, L. A., Faddeev, L. D., Quantization of Lie groups and Lie algebras, Leningrad Math. J., 1 (1990), 193-225.

[Sb] Selberg, A., Bemerkninger om et multipelt integral, Norsk Mat. Tidsskr., 26 (1994), 7178 ; (Coll. Papers, 1, 204-213).

[S1] Stokman, J. V., Multivariable big and little $q$-Jacobi polynomials, SIAM J. Math. Anal., 28 (1997), no. 2, 452-480.

[S2] _ Two limit transitions involving multivariable BC type Askey-Wilson polynomials, in : Quantum Groups and Quantum Spaces, eds. R. Budzyński et al., Banach Center Publ., 40 (1997), 415-428.

[S3] _ Multivariable BC type Askey-Wilson polynomials with partly discrete orthogonality measure, Ramanujan J., 1 (1997), 275-297.

[S4] _ On BC type basic hypergeometric orthogonal polynomials, preprint (1997), to 
appear in Trans. Amer. Math. Soc.

[SD] Stokman, J. V., Dijkhuizen, M. S., Quantized flag manifolds and irreducible *-representations, Comm. Math. Phys., 203 (1999), 297-324.

[SK] Stokman, J. V., Koornwinder, T. H., Limit transitions for BC type multivariable orthogonal polynomials, Can. J. Math., 49 (1997), 373-404.

[Su Sugitani, T., Zonal spherical functions on quantum Grassmann manifolds, preprint (1996).

[VS1] Vaksman, L. L., Soibelman, Ya. S., Algebra of functions on the quantum group SU(2), Funct. Anal. Appl., 22 (1988), 170-181.

[VS2] _ The algebra of functions on the quantum group $S U(n+1)$, and odddimensional quantum spheres, Leningrad Math. J., 2 (1991), 1023-1042.

[Vr] Vretare, L., Formulas for elementary spherical functions and generalized Jacobi polynomials, SIAM J. Math. Anal., 15 (1984), 805-833. 